\title{
The Split Delivery Vehicle Routing Problem with Time Windows and Customer Inconvenience Constraints
}

\author{
Nicola Bianchessi \\ Chair of Logistics Management, Gutenberg School of Management and Economics, Johannes Gutenberg University, \\ Jakob-Welder-Weg 9, D-55128 Mainz, Germany. nbianche@uni-mainz.de \\ Michael Drexl \\ Chair of Logistics Management, Gutenberg School of Management and Economics, Johannes Gutenberg University, \\ Jakob-Welder-Weg 9, D-55128 Mainz, Germany. \\ Faculty of Applied Natural Sciences and Industrial Engineering, Deggendorf Institute of Technology, D-94469 Deggendorf, \\ Germany. michael.drexl@th-deg.de \\ Stefan Irnich \\ Chair of Logistics Management, Gutenberg School of Management and Economics, Johannes Gutenberg University, \\ Jakob-Welder-Weg 9, D-55128 Mainz, Germany. irnich@uni-mainz.de \\ In classical routing problems, each customer is visited exactly once. By contrast, when allowing split deliver- \\ ies, customers may be served through multiple visits. This potentially results in substantial savings in travel \\ costs. Even if split deliveries are beneficial to the transport company, several visits may be undesirable on the \\ customer side: at each visit the customer has to interrupt his primary activities and handle the goods receipt. \\ The contribution of the present paper consists in a thorough analysis of the possibilities and limitations of \\ split delivery distribution strategies. To this end, we investigate two different types of measures for limiting \\ customer inconvenience (a maximum number of visits and the temporal synchronization of deliveries) and \\ evaluate the impact of these measures on carrier efficiency by means of different objective functions (compris- \\ ing variable routing costs, costs related to route durations, fixed fleet costs). We consider the vehicle routing \\ problem with time windows in which split deliveries are allowed (SDVRPTW) and define the corresponding \\ generalization that takes into account customer inconvenience constraints (SDVRPTW-IC). We design an \\ extended branch-and-cut algorithm to solve the SDVRPTW-IC and report on experimental results showing \\ the impact of customer inconvenience constraints. We finally draw useful insights for logistics managers on \\ the basis of the experimental analysis carried out. \\ Key words: Split delivery vehicle routing problem, Time windows, Synchronization, Maximum number of \\ visits, Branch-and-cut \\ History: Submitted on September 27, 2017. First revision submitted on February 23, 2018. Second revision \\ submitted on May 7, 2018.
}

\section{Introduction}

In classical routing problems concerning the delivery of goods, each customer is visited exactly once. By contrast, when allowing split deliveries, customers may be served by means of multiple 
visits. This potentially results in substantial savings in travel costs and fleet size, as in the split delivery vehicle routing problem (SDVRP), the relaxation of the vehicle routing problem (VRP) in which split deliveries are possible (see Archetti and Speranza (2012) and Irnich et al. (2014) for recent surveys on the topic). The option of split deliveries is clearly beneficial to the transport company. On the customer side, though, several visits cause inconvenience, as at each visit, the customer has to interrupt his primary activities to handle the goods receipt.

In the paper at hand, we introduce generalizations of the SDVRP that allow to control the degree of inconvenience caused by split deliveries and to balance overall distribution costs and customer satisfaction. This creates a win-win situation for transport companies and their customers. We examine two measures for limiting customer inconvenience:

(i) Maximum number of visits: this is the obvious and most direct way to limit customer inconvenience.

(ii) Temporal synchronization of deliveries: it is required that all deliveries to the same customer arrive within a pre-defined time span.

Maximum Number of Visits When a customer's demand exceeds the vehicle capacity, this customer is certainly split, so that the minimum number of visits to any customer is $n_{i}^{\min }=\left\lceil d_{i} / Q\right\rceil$ (where $d_{i}$ is the demand of customer $i$ and $Q$ the vehicle capacity). Archetti et al. (2006b) compare different VRP variants that result from fixing the number of visits to this minimum. Let $\mathrm{VRP}^{+}$be the variant in which each customer $i$ is visited exactly $n_{i}^{\min }$ times, where for $n_{i}^{\min }>1$ the demand $d_{i}$ can be arbitrarily split among the $n_{i}^{\min }$ visits. The authors show that, compared to the optimal $\mathrm{VRP}^{+}$solution, cost savings of $50 \%$ are possible when allowing an arbitrary number of visits, and that this bound is tight. By allowing more than the minimum number of visits, a large number of intermediate SDVRP variants can be defined, all with the purpose of controlling the possible customer inconvenience: for each customer $i$, the number of visits to this customer can be bounded above by $n_{i}^{\max } \geq n_{i}^{\min }$. Moreover, one may limit the overall number of visits to $n^{\max }$ for any $n^{\max } \geq \sum_{i} n_{i}^{\min }$ in order to reduce customer inconvenience.

Salazar-González and Santos-Hernández (2015) introduce the split-demand one-commodity pickup-and-delivery traveling salesman problem (SD1PDTSP), a very general problem that, despite its name, encompasses the multi-vehicle SDVRP as well as several other capacitated and uncapacitated routing problems without time windows as special cases. The authors propose a compact formulation for the SD1PDTSP and model the requirement of a maximum number of visits in the underlying network, by creating $n_{i}^{\max }$ vertices for each customer $i$.

Temporal Synchronization of Deliveries In this paper, we introduce synchronized deliveries as an alternative measure to reduce customer inconvenience. For this purpose, we embed synchronization constraints into a new split delivery routing problem which guarantees that all split deliveries 
Bianchessi, Drexl, and Irnich: The SDVRPTW and Customer Inconvenience Constraints

occurring to a customer must take place in a time interval of a given maximum duration. As the time dimension is relevant then, we focus on the split delivery vehicle routing problem with time windows (SDVRPTW), which is the split-delivery relaxation of the vehicle routing problem with time windows (VRPTW, Desaulniers et al. 2014). The variant of the SDVRPTW in which synchronization constraints are embedded is denoted by SDVRPTW-S; it is a special case of the more general SDVRPTW-IC that we formally define in Section 3.

In specific applications, when facilities to handle deliveries are scarce resources (e.g. a limited number of ramps or limited parking space), synchronization may aggravate conflicts. However, the typical split-delivery context is LTL transports for general cargo (deliveries of several pallets, trolleys, containers, and bulk load), which is not delivered ex curb. Then, parking is not an issue and synchronization can be applied without raising conflicts.

To increase the quality of service, a measure similar to the temporal synchronization of deliveries is considered in the consistent VRP (ConVRP), which has been introduced by Groër et al. (2009): over a planning horizon of several days, the same driver has to visit the same customers on each day these customers need service. No split delivery may occur. For each customer, it is required to synchronize the times of the visits on the different service days.

Minimum Delivery Amounts When trying to minimize customer inconvenience, what counts from the customer's point of view is the number of interruptions of his primary activities, in other words, the number of visits. A third way to reduce the number of interruptions is to require that split deliveries are allowed only if a minimum fraction of the customer's demand is delivered at each visit. Gulczynski et al. (2010) consider a pertinent generalization of the SDVRP. Besides defining a heuristic method for solving the problem, the authors give bounds for a worst-case SDVRP-MDA scenario. Their results are extended in Xiong et al. (2013). In the context of routing problems with profits, the idea of allowing to serve a customer by means of multiple visits only if a minimum fraction of the customer's demand is served at each visit is further examined by Wang et al. (2014). We do not consider the option of specifying minimum delivery amounts in our study, for two reasons. First, minimum delivery amounts are only an indirect way to achieve the primary goal of limiting the number of visits. It is simpler and more intuitive to set such a number directly. Second, and even more importantly, a minimum delivery amount does not make sense when the service times at customers can be assumed to be independent of the amount delivered. Judging from our experience, this is the case in many (though not all) real-world situations; moreover, it is a common assumption in the literature on the SDVRPTW as reviewed in the next paragraph.

To our knowledge, the most effective exact algorithms for the solution of the SDVRPTW are the branch-and-price-and-cut algorithms proposed by Archetti et al. (2011b) and Luo et al. (2016) 
(which are based on the work of Desaulniers 2010), and the branch-and-cut algorithm proposed by Bianchessi and Irnich (2018). The cited solution approaches are able to solve slightly different subsets of the SDVRPTW benchmark instances. However, concerning the number of instances solved to optimality, the branch-and-cut algorithm proposed in (Bianchessi and Irnich 2018) is superior, solving $5 \%$ more instances than the other solution approaches. In this work, we extend this branch-and-cut algorithm to address the different special cases of the SDVRPTW-IC.

The contribution of this paper is not only innovative from a methodological point of view. Even more importantly, we shed light on complex interdependencies between VRPTW, SDVRPTW, and SDVRPTW-IC special cases. Indeed, straightforward comparisons carry the danger of not taking all relevant effects into account. The standard SDVRPTW objective is the minimization of the variable routing costs (Desaulniers 2010). The most important insight gained from our experiments with the SDVRPTW-IC is that an exclusive comparison on the basis of variable routing costs is insufficient. Overall logistics costs surely depend on

(i) variable routing costs,

(ii) costs related to route durations, and

(iii) costs of the employed fleet,

and these cost elements should be included in a meaningful study analyzing savings that result from split deliveries.

To underline this statement, we present, at this early stage, the following brief computational comparison of VRPTW and SDVRPTW solutions. We used the well-known benchmark set of Solomon (1987), both as VRPTW and SDVRPTW instances. The set includes 56 instances, each of which comprises 100 customers. In order to keep the computational effort manageable, we considered only the smaller-sized instances constructed with the subsets of the first 25 and 50 customers respectively. However, as always done for the SDVRPTW, the vehicle capacity $Q$ is varied $(Q=30,50$ and 100) leading to $3 \cdot 2 \cdot 56=336$ instances (more details are provided in Section 5). With the standard objective of minimizing the variable routing costs and the branchand-cut that will be presented in Section 4, we obtained the results summarized in Table 1. The

Table 1 VRPTW and SDVRPTW solutions and comparison

\begin{tabular}{|c|c|c|c|c|c|c|c|c|c|c|}
\hline \multicolumn{2}{|c|}{ Instances } & \multicolumn{2}{|c|}{ VRPTW } & \multicolumn{2}{|c|}{ SDVRPTW } & \multicolumn{5}{|c|}{ Comparison } \\
\hline$n$ & $\overline{\#}$ & Feas. & Opt. & Feas. & Opt. & $\#$ & $\begin{array}{r}\text { Rout. Costs } \\
(\downarrow /=)\end{array}$ & $\begin{array}{l}\text { Durations } \\
(\downarrow /=/ \uparrow)\end{array}$ & $\begin{array}{r}\text { \#Vehicles } \\
(\downarrow /=)\end{array}$ & $\begin{array}{r}\text { Dominating } \\
\text { (Pareto) }\end{array}$ \\
\hline 25 & 168 & 135 & 135 & $\overline{16}$ & 168 & 135 & $56 / 79$ & $10 / 79 / 46$ & $8 / 127$ & 10 out of 135 \\
\hline 50 & 168 & 112 & 66 & 168 & 95 & 64 & $39 / 25$ & $8 / 25 / 31$ & $1 / 63$ & 8 out of 64 \\
\hline Total & 336 & 247 & 201 & 336 & 263 & 199 & $95 / 104$ & $18 / 104 / 77$ & $9 / 190$ & 18 out of 199 \\
\hline
\end{tabular}


Bianchessi, Drexl, and Irnich: The SDVRPTW and Customer Inconvenience Constraints

columns Feas. and Opt. show the number of instances for which a feasible VRPTW solution exists (recall that the capacity $Q$ is lowered compared to Solomon's definition) and for which both an optimal VRPTW and an optimal SDVRPTW solution were computed. Only the instances solved to optimality as VRPTW and as SDVRPTW were considered in the comparison. For these 199 instances, the section Comparison shows the number of instances in which the SDVRPTW solution improved $(\downarrow)$ the corresponding VRPTW solution w.r.t. variable routing costs (Rout. Costs), route durations (Durations), called "schedule times" in the work of Solomon (1987), and the number of vehicles employed (\#Vehicles). Recall that the routing costs of the SDVRPTW solution cannot increase but may stay constant (=). In our experiments, the SDVRPTW solution did never employ more vehicles than the corresponding VRPTW solution (this is why there are only the two cases $\downarrow$ and $=$ in column \#Vehicles). Dominating SDVRPTW solutions (their number is reported as Dominating) are those for which one of the three criteria is strictly improved while the others are not worse.

Beyond the numbers reported in Table 1, there are some important findings:

(i) For only 7 of the 199 instances, the variable routing costs are reduced by more than $1.5 \%$.

(ii) For 171 instances, the variable routing costs remained the same or were reduced by less than $0.5 \%$.

(iii) For the 9 instances for which the number of vehicles decreased, it decreased by 1 .

Additionally, Figure 1 quantifies, for the 95 instances for which variable routing costs decreased, the relationship between savings in variable routing costs and deviations of the route durations. To integrate the third criterion, we distinguish between SDVRPTW solutions that save (at least) one vehicle and all other solutions. The figures seem to indicate that, in many cases, even a rather small reduction in variable routing costs leads to a notable increase of the route durations. Recall, however, that such a statement is based on a limited set of benchmark problems and, more seriously, route durations and required fleet size are just an outcome of a pure variable routing costs minimization. We draw the following conclusions from the presented comparison of VRPTW and SDVRPTW:

(i) As the scientific VRP literature has not yet studied the full interdependency between all relevant cost types, a new SDVRPTW model should consider cost components related to route durations, such as driver wages, and fleet-related costs in addition to variable routing costs. This provides a more complete picture of the overall logistics costs and allows managers to better foresee the consequences of a possible change of the delivery strategy.

(ii) The incorporation of constraints that reduce customer inconvenience creates a variety of VRP models, for which VRPTW and SDVRPTW are the extreme cases. It is necessary to study these variants with the aim to better understand the impact of the different inconvenience constraints on the relevant cost types. 
(a) Instances with $n=25$ customers

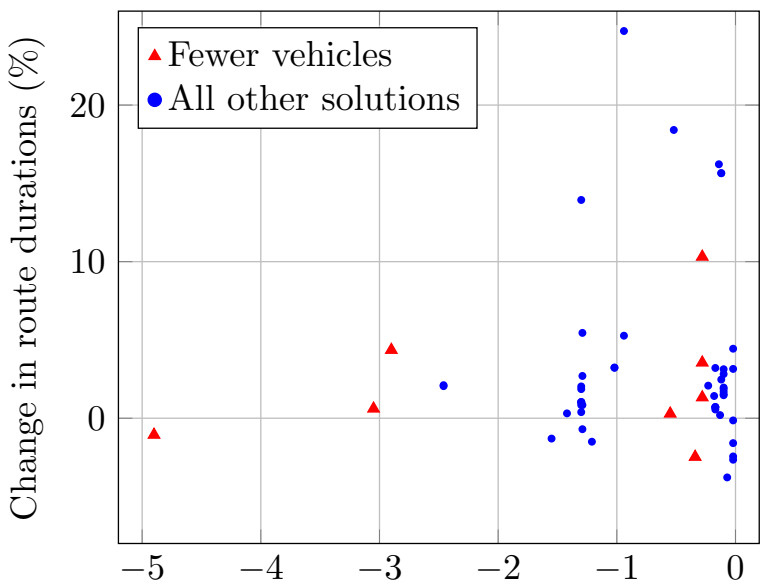

Change in variable routing costs $(\%)$ (b) Instances with $n=50$ customers

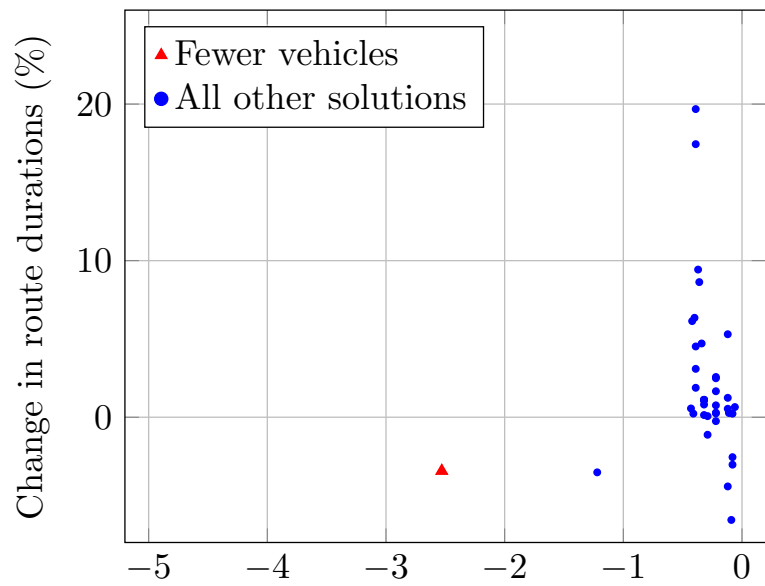

Change in variable routing costs $(\%)$

Figure 1 SDVRPTW vs. VRPTW solutions: relationship between savings in variable routing costs, change of route durations, and reduction of the number of routes.

(iii) For the Solomon-based SDVRPTW benchmark set, we have seen that the decrease in routing costs is only marginal compared to an offered $50 \%$ savings discussed in worst-case analyses. It is known that the savings from split deliveries mainly depend on the demand distribution (Archetti et al. 2006b). Without specific patterns for the customers' demand realizations, the Solomon-based benchmarks lack generality. We therefore create a new benchmark set in which groups of instances are characterized by different demand distributions (see Section 5.1).

The remainder of the paper is organized as follows. In Section 2, we formally define the SDVRPTW and list some important properties of the problem. A mathematical model for the SDVRPTW-IC is then discussed in Section 3. In Section 4, we present the branch-and-cut algorithm designed to solve the SDVRPTW-IC. Based on the experimental results obtained, we present in Section 5 the analysis of the impact of inconvenience constraints. Final conclusions are drawn in Section 6.

\section{The SDVRPTW and Properties of Optimal Solutions}

Let us first recall the definition of the SDVRPTW. The problem can be defined on a directed graph $G=(V, A)$, with vertex set $V$ and arc set $A$. The vertex set $V$ contains vertices 0 and $n+1$, representing the depot at the beginning and the end of the planning horizon respectively, and the set $N=\{1, \ldots, n\}$ representing the $n$ customers. Each customer $i \in N$ is associated with a positive demand $d_{i}$ that must be delivered by means of one or more visits within a prescribed time window $\left[e_{i}, l_{i}\right]$. Each delivery at customer $i$ must start within $\left[e_{i}, l_{i}\right]$, but a vehicle may arrive prior to 
$e_{i}$ and then wait until $e_{i}$ before starting the delivery. Moreover, a time window $\left[e_{0}, l_{0}\right]=\left[e_{n+1}, l_{n+1}\right]$ is associated with the depot to model the planning horizon. Each arc $(i, j) \in A$ represents the possibility to move from the location corresponding to vertex $i$ to the location corresponding to vertex $j$, and it is associated with a non-negative travel time $t_{i j}$ and a non-negative routing $\operatorname{cost} c_{i j}$. In particular, $t_{i j}$ includes the service time at $i$. We assume that the service time is constant for each visit and independent of the amount delivered. For each pair of vertices $i, j \in V, i \neq j$, there exists an $\operatorname{arc}(i, j) \in A$ if $e_{i}+t_{i j} \leq l_{j}$. We assume that all customer time windows are reduced so that $e_{i} \geq e_{0}+t_{0 i}$ and $l_{i} \leq l_{n+1}-t_{i, n+1}$ holds for all $i \in N$. As is common, the set $A$ includes the arc $(0, n+1)$, associated with zero travel time and routing cost, that allows modeling an idle vehicle, but not the arc $(n+1,0)$. A fleet $K$ of $|K|$ identical vehicles with a capacity $Q$ is available to serve the customers. The vehicles are initially located at the depot. A route corresponds to a path from 0 to $n+1$ in $G$. A route is feasible if the total demand delivered at the visited customers does not exceed the vehicle capacity and the time windows are respected. The SDVRPTW consists of determining a set of least-cost feasible routes such that all customer demands are met.

Given the above definitions and assumptions, and further assuming that the triangle inequality holds for routing costs and travel times, it is possible to prove that, for any SDVRP(TW) instance that has an optimal solution, there exists an optimal solution with the following properties:

Property 1. Two routes share at most one split customer (Dror and Trudeau 1990).

Property 2. Each arc between two vertices representing customers is traversed at most once (Gendreau et al. 2006).

Property 3. For each pair of reverse arcs between two customers at most one of them is traversed (Desaulniers 2010).

Property 4. All routes are elementary (Desaulniers 2010).

If, in addition, the vehicle capacity $Q$ and all demands $d_{i}$ for $i \in N$ are integer, then there exists an optimal solution to the SDVRPTW fulfilling Properties 1-4 and

Property 5. All delivery quantities are positive integers (Archetti et al. 2006a, 2011a).

These properties are exploited in the branch-and-cut algorithm that we present in Section 4.

\section{The SDVRPTW with Customer Inconvenience Constraints}

The SDVRPTW-IC is the generalization of the SDVRPTW taking into account upper bounds on the number of visits, and synchronization constraints for split deliveries occurring to the same customer. More formally, the following parameters become part of the problem definition:

Maximum number of visits: $n_{i}^{\max }$ and $n^{\max }$ limit the number of visits to $i \in N$ and the overall number of visits respectively; 
Temporal synchronization of deliveries: $\Delta_{i}$ limits the length of the time interval in which all deliveries to $i \in N$ must take place.

Moreover, the impact of these customer inconvenience constraints on the following types of distribution costs is taken into account in the SDVRPTW-IC objective function:

Variable routing costs: These are given for each arc $(i, j) \in A$ and are denoted by $c_{i j}$. They may also include a penalty $p_{i}$ when a customer $i \in N$ is visited. In this case, $\sum_{i \in N} n_{i}^{\min } p_{i}$ is the unavoidable penalty.

Costs related to route durations: We denote by $\gamma$ the time-to-cost ratio that, multiplied by the duration of a route, yields the duration-related costs.

Fixed vehicle costs: The fixed costs for using a vehicle are denoted by $C$.

We now describe two important characteristics of SDVRPTW-IC solutions.

Proposition 1. Given an SDVRPTW-IC instance fulfilling the assumptions made in Section 2. If this instance has an optimal solution, and if both routing costs and travel times satisfy the triangle inequality, the following two properties hold:

(a) There exists an optimal solution fulfilling Properties 1-4.

(b) If the vehicle capacity $Q$ and all demands $d_{i}$ for $i \in N$ are integer, then there exists an optimal solution fulfilling Properties 1-5.

Proof: (a) The proof of Property 1 is analogous to the one given by Gendreau et al. (2006) for the SDVRPTW, which, in turn, is based on the one by Dror and Trudeau (1990) for the SDVRP. Properties 2 and 3 follow immediately from Property 1. Given the above assumptions, Property 4 is fulfilled because a feasible SDVRPTW-IC solution with a non-elementary route that visits a customer more than once remains feasible with non-increased costs if all but the last visit to this customer are removed.

(b) The proof of this property is analogous to the one given by Archetti et al. (2006a) for the SDVRP.

We remark that, as Gulczynski et al. (2010) have shown, these properties are no longer fulfilled when minimum delivery amounts are specified.

It is anything but straightforward to develop a practicable and computationally attractive compact formulation for the SDVRPTW-IC. Bianchessi and Irnich (2018) have analyzed the difficulties of devising one for the SDVRPTW. Their arguments apply just as well to the SDVRPTW-IC and shall thus be briefly discussed in the following. First, as customers can be visited by several 
vehicles, it is impossible to attach unique resource variables to the vertices, e.g., variables indicating the accumulated customer demand and the service time. Consequently, formulations using Miller-Tucker-Zemlin types of constraints for the update of resource variables (see Miller et al. 1960) are not directly applicable in the split-delivery context. Second, using a three-index formulation, i.e., variables with vehicle indices, is not practicable either, as the resulting symmetries make any known branching scheme ineffective. Symmetry-breaking constraints (see, e.g. Fischetti et al. 1995) can only mitigate the negative effects of symmetry. Third, the formulation proposed by van Eijl (1995) for the delivery man problem and the one by Maffioli and Sciomachen (1997) for the sequential ordering problem show that resource variables may be associated with arcs. However, even if we can exploit Property 2 and associate time variables with arcs between customers, the problem remains that arcs between depot and customers (or vice versa) may be traversed by more than one vehicle. Hence, no time variables that uniquely define the vehicle travel times can be associated with these arcs.

Notwithstanding the above objections, we subsequently present a three-index model for the SDVRPTW-IC fulfilling Properties 2-4. Because of the mentioned weaknesses of such a formulation, however, we do not try to solve this model directly. Its purpose is solely to give a complete formal description of the SDVRPTW-IC. Our solution approach to the SDVRPTW-IC is based on a relaxed compact formulation using two-index variables and is described in the next section. In both models, we do not require Property 1, because this property cannot well be formulated with linear constraints. Moreover, Property 5 is fulfilled whenever a basic solution to an instance with integer demands and vehicle capacity is given.

The following model can be seen as a multi-commodity network flow formulation with additional variables and constraints, with a commodity for each available vehicle. The formulation uses

(i) binary flow variables $x_{i j}^{k}$ equal to 1 if vehicle $k \in K$ travels along arc $(i, j) \in A$, and 0 otherwise;

(ii) non-negative continuous flow variables $T_{i}^{k}$ representing the start of service of vehicle $k \in K$ when visiting vertex $i \in N$;

(iii) non-negative continuous variables $\delta_{i}^{k}$ representing the quantity delivered by vehicle $k \in K$ to customer $i \in N$;

(iv) continuous variables $E_{i}$ representing the earliest start of service at customer $i \in N$.

The symbols $\Gamma^{+}(S)$ and $\Gamma^{-}(S)$ respectively denote the forward and backward star of $S \subseteq N$. For simplicity, we use $\Gamma^{+}(i)$ and $\Gamma^{-}(i)$ whenever $S=\{i\}$. Moreover, we define $A(N)=\{(i, j) \in A: i \in$ $N, j \in N\}$.

The multi-commodity flow formulation for the SDVRPTW-IC is as follows:

$$
\min \sum_{k \in K}\left(\sum_{(i, j) \in A} c_{i j} x_{i j}^{k}+\gamma\left(T_{n+1}^{k}-T_{0}^{k}\right)+C \sum_{i \in N} x_{0 i}^{k}\right)
$$




$$
\begin{aligned}
& \text { s.t. } \quad \sum_{(0, j) \in \Gamma^{+}(0)} x_{0 j}^{k}=\sum_{(i, n+1) \in \Gamma^{-}(n+1)} x_{i, n+1}^{k}=1 \quad k \in K \\
& \sum_{(h, i) \in \Gamma^{-}(i)} x_{h i}^{k}-\sum_{(i, j) \in \Gamma^{+}(i)} x_{i j}^{k}=0 \quad i \in N, k \in K \\
& x_{i j}^{k}\left(T_{i}^{k}+t_{i j}-T_{j}^{k}\right) \leq 0 \quad(i, j) \in A, k \in K \\
& e_{i} \sum_{(i, j) \in \Gamma^{+}(i)} x_{i j}^{k} \leq T_{i}^{k} \leq l_{i} \sum_{(i, j) \in \Gamma^{+}(i)} x_{i j}^{k} \quad i \in N, k \in K \\
& \sum_{k \in K} \delta_{i}^{k} \geq d_{i} \quad i \in N \\
& 0 \leq \delta_{i}^{k} \leq \min \left\{d_{i}, Q\right\} \sum_{(i, j) \in \Gamma^{+}(i)} x_{i j}^{k} \quad i \in N, k \in K \\
& \sum_{i \in N} \delta_{i}^{k} \leq Q \quad k \in K \\
& x_{i j}^{k} \in\{0,1\} \quad(i, j) \in A, k \in K
\end{aligned}
$$

Additional constraints enforcing Properties 2 and 3 are added:

$$
\begin{array}{ll}
\sum_{k \in K} x_{i j}^{k} \leq 1 & (i, j) \in A(N) \\
\sum_{k \in K} x_{i j}^{k}+x_{j i}^{k} \leq 1 & (i, j),(j, i) \in A(N): i<j
\end{array}
$$

Constraints to alleviate customer inconvenience are:

$$
\begin{array}{ll}
\sum_{k \in K} \sum_{(i, j) \in \Gamma^{+}(i)} x_{i j}^{k} \leq n_{i}^{\max } & i \in N \\
\sum_{k \in K} \sum_{i \in N} \sum_{(i, j) \in \Gamma^{+}(i)} x_{i j}^{k} \leq n^{\max } & \\
E_{i} \leq T_{i}^{k}+l_{i}\left(1-\sum_{(i, j) \in \Gamma^{+}(i)} x_{i j}^{k}\right) & i \in N, k \in K \\
T_{i}^{k} \leq E_{i}+\Delta_{i} & i \in N, k \in K
\end{array}
$$

The objective function (1a) calls for the minimization of the total variable routing costs, the costs related to route durations, and the fixed costs for employing vehicles. Constraints (1b) and (1c) impose the route associated with each vehicle to be a $0-(n+1)$-path. Feasibility regarding time-window constraints and elementarity of the routes is guaranteed by (1d) and (1e). Clearly, constraints $(1 \mathrm{~d})$ can be linearized by $T_{i}^{k}+t_{i j}-T_{j}^{k} \leq M_{i j}\left(1-x_{i j}^{k}\right)$, where $M_{i j}$ is an arc-specific large constant, e.g., $M_{i j}=\max \left\{l_{i}+t_{i j}-e_{j}, 0\right\}$. Constraints (1f) ensure customer demands are met. Constraints (1g) allow a vehicle to deliver only to visited customers and (1h) are the capacity constraints. The domain of the vehicle flow variables is defined by constraints (1i). By setting 
Bianchessi, Drexl, and Irnich: The SDVRPTW and Customer Inconvenience Constraints

Article submitted to Transportation Science; manuscript no. TS-2017-0288.R2

duration-related and fixed costs $\gamma=C=0$, the system (1a)-(1i) is the basic vehicle-indexed formulation of the SDVRPTW. Desaulniers (2010) strengthens this formulation by adding tighter bounds on the fleet size, capacity cuts, and 2-path cuts. We explain these cuts later in the context of our branch-and-cut approach in Section 4.2.

Constraints $(1 \mathrm{j})$ and $(1 \mathrm{k})$ come from Property 2 and 3 respectively. They are redundant for model (1a)-(1i), but will turn out helpful in our new compact model.

Constraints (11)-(1o) reduce or eliminate customer inconvenience caused by deferred and multiple visits. Constraints (1l) and (1m) limit the maximum number of visits to customers, individually and in total. Temporal synchronization of visits is guaranteed by constraints (1n) and (1o), where $\Delta_{i}=0$ imposes simultaneous deliveries and $\Delta_{i}=l_{i}-e_{i}$ allows to spread them arbitrarily in the service time window.

\section{A Branch-and-Cut Algorithm}

In this section, we extend the branch-and-cut algorithm proposed by Bianchessi and Irnich (2018) to address the SDVRPTW-IC. The algorithm is based on a compact formulation that in fact constitutes a relaxation of the problem. This means that some integer solutions to the relaxed formulation are infeasible for the SDVRPTW-IC. Valid inequalities are used in order to strengthen the relaxed compact formulation and possibly cut off solutions that are infeasible for the SDVRPTW-IC. However, even with the valid inequalities, integer solutions to the new compact formulation remain to be tested for feasibility. The positive arc flow values in any given integer solution to the relaxed formulation induce a subnetwork of the original instance. As there are only few split customers in a typical solution, such a subnetwork will regularly contain only few arcs. Hence, all time-window feasible routes on this subnetwork can be enumerated. An extended set-covering problem is then solved in order to decide on the selection of routes, their schedules, the quantities to deliver to the visited customers, and, hence, overall feasibility. All solutions proved infeasible are cut off from the feasible region of the relaxed problem.

In Section 4.1, we define the relaxed compact formulation for the SDVRPTW-IC and show how an optimal solution to this formulation may not be feasible to the original problem. In Section 4.2, we summarize the valid inequalities used in order to strengthen the relaxed formulation and cut off solutions that are infeasible for the SDVRPTW-IC. Finally, in Section 4.3, we present the feasibility-checking procedure and the feasibility cuts.

\subsection{Relaxed Compact Formulation}

The relaxed compact formulation for the SDVRPTW-IC is a two-commodity flow formulation with additional variables and constraints. The first commodity represents the available vehicles and the second represents the service times imposed by the routes. The formulation uses 
(i) integer variables $z_{i}$ indicating the number of times vertex $i \in N$ is visited by the vehicles;

(ii) integer flow variables $x_{i j}$ indicating the flow of the vehicles along arc $(i, j) \in A$;

(iii) non-negative continuous flow variables $T_{i j}$ indicating the service start time at $i \in N$ when a vehicle travels directly from $i$ to $j \in N$; moreover, $T_{0 i}$ is the sum of the departure times at the depot 0 of the vehicles traveling along $(0, i)$, and $T_{i, n+1}$ is the sum of the service start times at customer $i$ of the vehicles traveling along $(i, n+1)$ (the latter type of variables is not needed for pure SDVRPTW but indispensable here due to the route durations related part of the objective);

(iv) non-negative continuous variables $w_{i j}$ indicating the waiting time at $j \in N$ when a vehicle travels directly from $i$ to $j$ for $(i, j) \in A(N)$;

(v) non-negative continuous variables $E_{i}$ representing the earliest service time at customer $i \in N$. In the remainder, we will refer to $T_{i j}$ and $w_{i j}$ as service-time and waiting-time flow variables respectively.

We use the following additional notation. We define $\Gamma_{N}^{+}(S)=\Gamma^{+}(S) \cap A(N)$ and $\Gamma_{N}^{-}(S)=$ $\Gamma^{-}(S) \cap A(N)$. Again, we write $\Gamma_{N}^{+}(i)$ and $\Gamma_{N}^{-}(i)$ for singleton sets $S=\{i\}$. Finally, we define $K_{S}=\left\lceil\sum_{i \in S} d_{i} / Q\right\rceil$ as the minimum number of vehicles required to serve customers in set $S \subseteq N$.

The relaxed two-commodity flow formulation for the SDVRPTW-IC is as follows:

$$
\begin{aligned}
& \min \sum_{(i, j) \in A} c_{i j} x_{i j}+\gamma\left(\sum_{(i, j) \in A} t_{i j} x_{i j}+\sum_{(i, j) \in A_{N}} w_{i j}\right)+C \sum_{(0, i) \in \Gamma_{N}^{+}(0)} x_{0 i} \\
& \text { s.t. } \quad \sum_{(h, i) \in \Gamma^{-}(i)} x_{h i}=\sum_{(i, j) \in \Gamma^{+}(i)} x_{i j}=z_{i} \quad i \in N \\
& \sum_{(0, j) \in \Gamma^{+}(0)} x_{0 j}=|K| \\
& \sum_{(i, j) \in \Gamma^{+}(S)} x_{i j} \geq K_{S} \quad S \subseteq N,|S| \geq 2 \\
& \sum_{(h, i) \in \Gamma^{-}(i)}\left(T_{h i}+t_{h i} x_{h i}\right)+\sum_{(h, i) \in \Gamma_{N}^{-}(i)} w_{h i}=\sum_{(i, j) \in \Gamma^{+}(i)} T_{i j} \quad i \in N \\
& e_{i} x_{i j} \leq T_{i j} \leq l_{i} x_{i j} \quad(i, j) \in A \\
& \max \left\{0, e_{j}-t_{i j}-l_{i}\right\} x_{i j} \leq w_{i j} \leq \max \left\{0, l_{j}-t_{i j}-e_{i}\right\} x_{i j} \quad(i, j) \in A(N) \\
& z_{i} \geq\left\lceil d_{i} / Q\right\rceil \text { and integer } \\
& i \in N \\
& x_{i j} \in\{0,1\} \\
& (i, j) \in A(N) \\
& x_{i j} \geq 0 \text { and integer }
\end{aligned}
$$

with customer inconvenience constraints

$$
z_{i} \leq n_{i}^{\max } \quad i \in N
$$


Bianchessi, Drexl, and Irnich: The SDVRPTW and Customer Inconvenience Constraints

$$
\begin{aligned}
& \sum_{i \in N} z_{i} \leq n^{\max } \\
& E_{i} \leq T_{i j}+l_{i}\left(1-x_{i j}\right) \\
& T_{i j} \leq E_{i}+\Delta_{i}
\end{aligned}
$$$$
(i, j) \in A(N)
$$

The objective function (2a) calls for the minimization of the total costs. Constraints (2b) impose flow conservation for the vehicle flow variables. (2c) is the fleet size constraint. Constraints $(2 \mathrm{~d})$ prevent the generation of paths not connected to the depot. Moreover, as shown by Bianchessi and Irnich (2018), (2d) are necessary but not sufficient for maintaining capacity constraints. Constraints $(2 \mathrm{e})-(2 \mathrm{~g})$ impose conservation for the service-time flow, ensure consistency among the $T_{i j}$, $w_{i j}$, and $x_{i j}$ variable values, and partially ensure time-window prescriptions. Constraints $(2 \mathrm{~h})-(2 \mathrm{j})$ define the domains for the integer variables. Note that the binary requirement in (2i) results from Property 2.

Constraints $(2 \mathrm{k})-(2 \mathrm{n})$ are the customer inconvenience constraints. (2k) explicitly specify an upper bound on the number of visits at each customer, and (2l) enforce a limit on the overall number of deliveries performed. $(2 \mathrm{~m})$ and $(2 \mathrm{n})$ are the synchronization constraints which guarantee that all visits to a customer $i$ are performed within the time interval $\Delta_{i}$.

An optimal solution to (2) may not be feasible for the SDVRPTW-IC. Bianchessi and Irnich (2018) discuss examples showing that an optimal solution to the relaxed formulation for the SDVRPTW can violate the capacity or time-window constraints. Those examples apply also to the SDVRPTW-IC. Consider the following

EXAmple 1. The instance depicted in Figure 2 shows that an integer solution to (2) can violate synchronization constraints even though it is feasible w.r.t. capacity and time-window constraints. In this instance, the depicted arcs have costs and travel times equal to 1, while all other arcs (not shown) have costs and travel times equal to 2 . The demands $d_{i}$ and the time windows $\left[e_{i}, l_{i}\right]$ of the $n=5$ customers are presented close to each customer $i \in\{1,2, \ldots, 5\}$. The depot time window is assumed to be non-constraining, i.e., $\left[e_{0}, l_{0}\right]=\left[e_{n+1}, l_{n+1}\right]=[0,10]$. The capacity of the vehicles is $Q=10$. The depicted arcs have a flow of 1 and form the unique optimal solution to the relaxed model (2). In fact, two fully loaded vehicles are required to serve the 5 customers and, due to the given customer demands, one of the customers must receive split deliveries. Therefore, the solution consists of two routes, for a total of 8 arcs. Selecting any set of arcs different from those depicted would increase the cost of the solution. As far as time-window prescriptions, demands, and vehicle capacity are concerned, this optimal solution can be converted into a feasible SDVRPTWIC solution, e.g., using the two routes $(0,1,3,4, n+1)$ and $(0,2,3,5, n+1)$. In the first route, the values of the service-time flow variables $T_{i j}$ with $i=3$ or $j=3$ are uniquely defined: $T_{13}=4$ and 
$T_{34}=5$. In the second route, different values are possible for the $T_{i j}$ variables. In particular, when customers are served as early as possible, then $T_{23}=1$ and $T_{35}=2$. If customers are served as late at possible, then $T_{23}=2$ and $T_{35}=3$. If $\Delta_{3} \geq 2$, then the corresponding SDVRPTW-IC solution with the as-late-as-possible schedule for the second route is feasible with regard to synchronization constraints (service times at customer 3 are then 5 and 3 and thus differ by not more than $\Delta_{3}$ ). However, if $\Delta_{3}=1$, then customer 3 cannot be served by routes $(0,1,3,4, n+1)$ and $(0,1,3,5, n+1)$ in such a way that synchronization constraints are satisfied in a feasible SDVRPTW-IC solution. Nevertheless, the assignments $T_{01}=3, T_{13}=4, T_{34}=4, T_{46}=5$ and $T_{02}=0, T_{23}=1, T_{35}=3$, $T_{56}=4$ to the service-time flow variables (and $\mathbf{w}=0$ for the waiting time variables) are feasible for model (2).

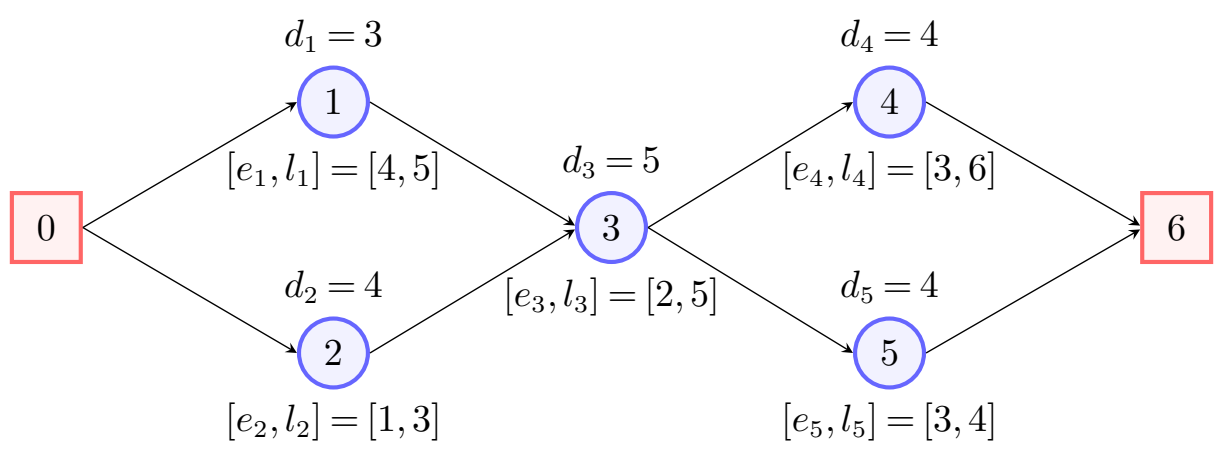

\section{Figure 2 Optimal solution to formulation (2) that is infeasible for the SDVRPTW-IC w.r.t. synchronization constraints.}

The above example has shown that the relaxed model (2) contains infeasible integer solutions w.r.t. the synchronization constraints of SDVRPTW-IC. When the minimization of the route durations becomes part of the objective, i.e., for $\gamma>0$, model (2) also contains integer solutions that are feasible w.r.t. routing but infeasible w.r.t. scheduling. In this case, the solution represented by values of the routing variables $x_{i j}$ can be converted into a feasible SDVRPTW-IC solution. However, such a feasible SDVRPTW-IC solution requires a different schedule than what the $T_{i j}$ variable values indicate. In consequence, model (2) evaluates the solution given by the $x_{i j}$ variables with a too small objective value, computed with an infeasible set of associated $T_{i j}$ variable values. In summary, formulation (2) is therefore a relaxation w.r.t. the routing and scheduling decisions as well as the objective function.

ExAmple 2. An example for such a relaxed solution is presented in Figure 3. Here, the only feasible SDVRPTW-IC solution comprises the routes $(0,1,3,4, n+1)$ and $(0,2,3,5, n+1)$. Due to duration minimization, the values $T_{01}=4, T_{13}=5, T_{34}=6, T_{46}=8$, and $w_{34}=1$ of the servicetime flow and waiting time variables in the first route are unique. For the second route, different 
sets of values can instead be assigned to the service-time flow and waiting time variables: When customers are served as early as possible, then $T_{02}=0, T_{23}=1, T_{35}=2$, and $T_{56}=3$. In contrast, when customers are served as late at possible, then $T_{02}=2, T_{23}=3, T_{35}=4$, and $T_{56}=5$. With both schedules, the second vehicle never waits along the second route. Hence, the overall waiting time is unique and given by $w_{34}=1$. In contrast, the values $T_{01}=4, T_{13}=5, T_{34}=7, T_{46}=8, w_{34}=0$ and $T_{02}=1, T_{23}=2, T_{35}=2, T_{56}=3$ of the service-time flow and waiting variables are feasible for the relaxed model (2). Here, no waiting seems to be necessary. The objective (2a) of the relaxed model underestimates the true SDVRPTW-IC costs for the feasible $x$-values by $\gamma>0$.

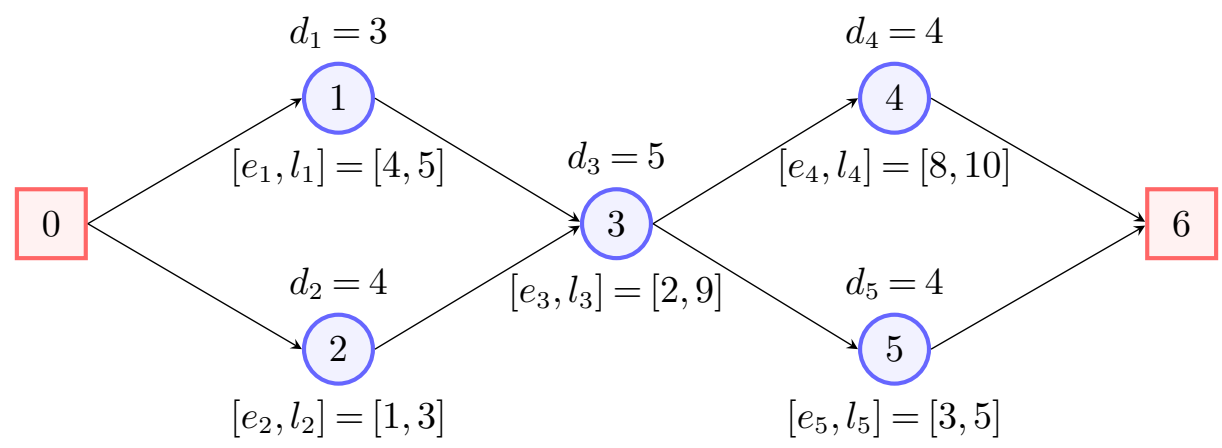

Figure 3 Optimal solution to formulation (2) in which the arc flow variables represent a set of feasible SDVRPTWIC routes. The objective (2a) however underestimates the true route durations and costs, because optimal values for the service-time and waiting flow variables in (2) are infeasible for the routes.

Note that model (2) can be reformulated without making use of the waiting time flow variables. Objective (2a) and constraints (2e) and $(2 \mathrm{~g})$ need to be replaced. The relaxed formulation becomes:

$$
\begin{aligned}
& \min \sum_{(i, j) \in A} c_{i j} x_{i j}+\gamma \sum_{(i, j) \in A} t_{i j} x_{i j}+\gamma \sum_{i \in N}\left(\sum_{(i, j) \in \Gamma^{+}(i)} T_{i j}-\sum_{(h, i) \in \Gamma^{-}(i)}\left(T_{h i}+t_{h i} x_{h i}\right)\right)+C \sum_{(0, i) \in \Gamma_{N}^{+}(0)} x_{0 i} \\
& \\
& \quad \sum_{(h, i) \in \Gamma^{-}(i)}\left(T_{h i}+t_{h i} x_{h i}\right) \leq \sum_{(i, j) \in \Gamma^{+}(i)} T_{i j} \\
& \quad \sum_{(h, i) \in \Gamma_{N}^{-}(i)} w_{h i}^{L B} x_{h i} \leq \sum_{(i, j) \in \Gamma^{+}(i)} T_{i j}-\sum_{(h, i) \in \Gamma^{-}(i)}\left(T_{h i}+t_{h i} x_{h i}\right) \leq \sum_{(h, i) \in \Gamma_{N}^{-}(i)} w_{h i}^{U B} x_{h i} i \in N \\
& (2 \mathrm{~b})-(2 \mathrm{~d}),(2 \mathrm{f}),(2 \mathrm{~h})-(2 \mathrm{n})
\end{aligned}
$$

where $w_{h i}^{L B}=\max \left\{0, e_{i}-t_{h i}-l_{h}\right\}$ and $w_{h i}^{U B}=\max \left\{0, l_{i}-t_{h i}-e_{h}\right\}$. As (3c) are the aggregate form of $(2 \mathrm{~g})$, the arising formulation is slightly weaker than (2). However, the new formulation (3) has $\mathcal{O}\left(n^{2}\right)$ fewer variables and constraints, and preliminary experiments showed this is beneficial from the computational point of view. Our branch-and-cut algorithm is therefore based on (3). 


\subsection{Valid Inequalities}

In classical branch-and-cut algorithms, valid inequalities are used to strengthen the formulation of the problem addressed. Since (3) is a relaxed formulation, in our algorithm valid inequalities are also used to cut off integer solutions to (3) that are infeasible for the SDVRPTW-IC.

We consider the same classes of valid inequalities as Bianchessi and Irnich (2018):

- Inequalities

$$
x_{i j}+x_{j i} \leq 1 \quad(i, j),(j, i) \in A(N): i<j,
$$

which can be imposed due to Property 3.

- Capacity cuts (2d) as stated in the previous section.

- 2-path cuts, introduced by Kohl et al. (1999):

$$
\sum_{(i, j) \in \Gamma^{+}(S)} x_{i j} \geq 2
$$

which apply whenever a subset $S \subseteq N$ of the customers cannot be served with a single vehicle.

- Connectivity cuts of the form

$$
\sum_{(i, j) \in \Gamma^{+}(S)} x_{i j} \geq z_{u} \quad S \subseteq N,|S| \geq 2, u \in S
$$

They prove useful even though already the capacity cuts ensure that any subset of customers is connected to the depot.

- Infeasible-path constraints and path-matching constraints, introduced by Bianchessi and Irnich (2018). These are two new classes of valid inequalities for the SDVRPTW. The former are an adaptation to the SDVRPTW of the cuts bearing the same name and introduced by Ascheuer et al. $(2000,2001)$. The latter are a generalization of the former involving several partial paths meeting at a specified customer vertex. It is straightforward to prove that both types of cuts are also valid for the SDVRPTW-IC. Since their definitions require extensive additional notation, their closed-form expressions are given in Section EC.1 of the e-Companion, together with the details concerning the corresponding separation procedures devised in (Bianchessi and Irnich 2018).

Inequalities (4) are added to the formulation right from the start, whereas the other cuts are dynamically separated in the course of the algorithm. We apply the same separation strategies as Bianchessi and Irnich (2018): only inequalities exceeding a violation of $\epsilon=0.05$ are inserted. The different classes of cuts are considered hierarchically, in the order they are presented in this section. This means that, if a violated inequality is found in a given class, the separation routines for the cuts further down the hierarchy are not called. At most 500 cuts are added in one run of the separation procedure. 
Bianchessi, Drexl, and Irnich: The SDVRPTW and Customer Inconvenience Constraints

Article submitted to Transportation Science; manuscript no. TS-2017-0288.R2

\subsection{Feasibility Checking}

Recall that every time a feasible integer solution to the relaxed formulation (3) is found, a procedure must check whether the solution is also feasible to the SDVRPTW-IC. If not, a feasibility cut must be inserted to cut off this solution from the feasible region of the relaxed problem.

The checking procedure we use is based on the one proposed by Bianchessi and Irnich (2018) and works as follows. Let $\bar{s}=(\overline{\mathbf{x}}, \overline{\mathbf{z}}, \overline{\mathbf{T}}, \overline{\mathbf{E}})$ be an integer solution to the relaxed formulation (3), possibly augmented by branching and cutting constraints. Let $\bar{Z}$ denote the costs of the solution.

For $\bar{V}=V$ and $\bar{N}=\bar{V} \backslash\{0, n+1\}$ we define a residual network $H(\bar{V}, \overline{\mathbf{x}})=(\bar{V}, \bar{A})$, with $\bar{A}=$ $\left\{(i, j) \in A: \bar{x}_{i j} \geq 1\right\} \cup\{(0, j): j \in \bar{N}\} \cup\{(i, n+1): i \in \bar{N}\}$. Furthermore, let $\bar{S}=\left\{i \in \bar{N}: \bar{z}_{i} \geq 2\right\}$ be the set of customers receiving split deliveries in solution $\bar{s}$ (split customers). For the non-split customers $i \in \bar{N} \backslash \bar{S}$, we know that the delivery quantity is identical to $d_{i}$ independently of the route serving the customer. Moreover, if Property 5 holds, the minimum delivery amount to split customers is equal to 1 . According to these minimum delivery amounts, we define $\bar{R}$ as the set of all elementary $0-(n+1)$-paths (routes) in $H(\bar{V}, \overline{\mathbf{x}})$ satisfying time-window and vehicle capacity constraints. We generate $\bar{R}$ by exploring $H(\bar{V}, \overline{\mathbf{x}})$ in a depth-first way.

An instance of the SDVRPTW-IC, defined on the basis of $\bar{V}$ and $\overline{\mathbf{x}}$ imposing the route set $\bar{R}$, can be modeled by a path-based formulation. Some additional notation is required. Let $\bar{N}(r) \subseteq \bar{N}$ be the subset of customers visited by route $r \in \bar{R}$. We distinguish between routes $\bar{R}^{s}$ visiting a single customer, i.e., routes of the form $(0, i, n+1)$ for $i \in N$, and routes $\bar{R}^{m}$ visiting more than one customer. Obviously, $\bar{R}=\bar{R}^{m} \cup \bar{R}^{s}$ and $\bar{R}^{m} \cap \bar{R}^{s}=\varnothing$.

The schedule of a route needs to be feasible regarding time-window and synchronization constraints. In order to guarantee a feasible schedule for the route $r \in \bar{R}$, it suffices to impose constraints on the visit times at the vertices $i \in V_{r}^{\text {time }}$, where $V_{r}^{\text {time }}$ is the set $(\bar{N}(r) \cap \bar{S}) \cup\{0, n+1\}$. We define the relation $P_{r}^{\text {time }}$ so that $(i, j) \in P_{r}^{\text {time }}$ if and only if $i, j \in V_{r}^{\text {time }}$ and $i$ is visited before $j$ in route $r$ with no other vertex of $V_{r}^{\text {time }}$ in between.

Extended Set-Covering Model The path-based formulation for the SDVRPTW-IC, defined relatively to $\bar{V}$ and $\overline{\mathbf{x}}$, uses then

(i) non-negative integer and binary variables $\lambda^{r}$ indicating the number of vehicles assigned to route $r \in \bar{R}^{s}$ and $\bar{R}^{m}$ respectively,

(ii) non-negative continuous variables $\delta_{i}^{r}$ indicating the quantity delivered to customer $i \in \bar{N}(r) \cap \bar{S}$ by route $r \in \bar{R}$,

(iii) non-negative continuous variables $T_{i}^{r}$ representing the service time at customer $i \in \bar{N}(r) \cap \bar{S}$, the departure time at the depot $i=0$, and the arrival time at the depot $i=n+1$ for route $r \in \bar{R}^{m}$, 
and it reads as follows:

$$
\begin{aligned}
& \bar{Z}_{\bar{R}}=\min \gamma \sum_{r \in \bar{R}^{m}}\left(T_{n+1}^{r}-T_{0}^{r}\right)+\gamma \sum_{\substack{r \in \bar{R}^{s} \\
r=(0, i, n+1)}}\left(t_{0 i}+t_{i, n+1}\right) \lambda^{r}+\sum_{r \in \bar{R}}\left(c^{r}+C\right) \lambda^{r} \\
& \text { s.t. } \quad \gamma \sum_{r \in \bar{R}^{m}}\left(T_{n+1}^{r}-T_{0}^{r}\right)+\gamma \sum_{\substack{r \in \bar{R}^{s}: \\
r=(0, i, n+1)}}\left(t_{0 i}+t_{i, n+1}\right) \lambda^{r}+\sum_{r \in \bar{R}}\left(c^{r}+C\right) \lambda^{r} \leq \bar{Z}^{*} \\
& \sum_{r \in \bar{R}: i \in \bar{N}(r)} \delta_{i}^{r} \geq d_{i} \quad i \in \bar{S} \\
& \sum_{r \in \bar{R}: i \in \bar{N}(r)} \lambda_{i}^{r} \geq 1 \quad i \in \bar{N} \backslash \bar{S} \\
& \sum_{i \in \bar{S} \cap \bar{N}(r)} \delta_{i}^{r}+\sum_{i \in(\bar{N} \backslash \bar{S}) \cap \bar{N}(r)} d_{i} \lambda_{i}^{r} \leq Q \lambda^{r} \quad r \in \bar{R} \\
& e_{i}^{r} \lambda^{r} \leq T_{i}^{r} \leq l_{i}^{r} \lambda^{r} \\
& r \in \bar{R}^{m}, i \in V_{r}^{\text {time }} \\
& T_{i}^{r}+t_{i j}^{r} \lambda^{r} \leq T_{j}^{r} \quad r \in \bar{R}^{m},(i, j) \in P_{r}^{\text {time }} \\
& \sum_{r \in \bar{R}}\left(b_{i j}^{r}+b_{j i}^{r}\right) \lambda^{r} \leq 1 \quad(i, j),(j, i) \in \bar{A}(\bar{N}), i<j \\
& \sum_{r \in \bar{R}} \lambda^{r} \leq|K| \\
& \delta_{i}^{r} \geq 0 \\
& r \in \bar{R}, i \in \bar{N}(r) \cap \bar{S} \\
& \lambda^{r} \in\{0,1\} \\
& r \in \bar{R}^{m} \\
& \lambda^{r} \geq 0 \text { and integer } \\
& r \in \bar{R}^{s}
\end{aligned}
$$

with customer inconvenience constraints

$$
\begin{array}{ll}
\sum_{r \in \bar{R}} \sum_{(i, j) \in \Gamma^{+}(i)} b_{i j}^{r} \lambda^{r} \leq n_{i}^{\max } & i \in N \\
\sum_{r \in \bar{R}} \sum_{(i, j) \in A: i \in N} b_{i j}^{r} \lambda^{r} \leq n^{\max } & \\
E_{i} \leq T_{i}^{r}+l_{i}\left(1-\lambda^{r}\right) & r \in \bar{R}^{m}, i \in \bar{N}(r) \cap \bar{S} \\
T_{i}^{r} \leq E_{i}+\Delta_{i} & r \in \bar{R}^{m}, i \in \bar{N}(r) \cap \bar{S}
\end{array}
$$

where $c^{r}$ are the variable routing costs of route $r \in \bar{R}, \bar{Z}^{*}$ is the upper bound to the SDVRPTW-IC stored in the branch-and-cut algorithm, $t_{i j}^{r}$ is the time required to travel (without waiting) from $i$ to $j$ along route $r$, if $(i, j) \in P_{r}^{\text {time }}$, and $b_{i j}^{r}$ is a binary arc indicator equal to 1 if $\operatorname{arc}(i, j) \in \bar{A}(\bar{N})$ is used in route $r \in \bar{R}, 0$ otherwise.

The objective function (7a) minimizes the costs of all routes in use. If model (7) is infeasible, we set $\bar{Z}_{\bar{R}}=\infty$. Constraints (7b) impose an upper bound on the objective value $\bar{Z}_{\bar{R}}$. Constraints (7c) and $(7 \mathrm{~d})$ ensure that customer demands are met. Vehicle capacity constraints are imposed by 
Bianchessi, Drexl, and Irnich: The SDVRPTW and Customer Inconvenience Constraints

(7e). Constraints (7f) and (7g) define the values of the service time variables associated with split customers. Property 3 implies constraints (7h). Constraint (7i) guarantees that the fleet size is respected. Finally, constraints (7j)-(7l) define the domains of the $\delta_{i}^{r}$ and $\lambda^{r}$ variables.

Concerning customer inconvenience constraints, $(7 \mathrm{~m})$ and $(7 \mathrm{n})$ limit the maximum number of visits to customers, individually and in total, and (7o) and (7p) impose synchronization of visits.

Note that constraints $(7 \mathrm{~b})-(7 \mathrm{l})$ do not impose that each arc $(i, j) \in \bar{A}$ be traversed exactly $\bar{x}_{i j}$ times by the selected routes. Moreover, $\bar{A}$ may include arcs in $\Gamma^{+}(0) \cup \Gamma^{-}(n+1)$ that are not used in solution $\bar{s}$. Alternative SDVRPTW-IC solutions are thus possible, and improving solutions are found whenever $\bar{Z}_{\bar{R}}<\bar{Z}$. In addition, customer visits with zero deliveries are possible in (7), i.e., $\lambda^{r}>0$ but $\delta_{i}^{r}=0$ for some $i \in \bar{N}(r) \cap \bar{S}$. Due to the validity of the triangle inequality and assuming that waiting time does not cost more that traveling time, improving (or at least not worse) alternative feasible solutions can be derived by removing customers with a delivery quantity of 0 from the routes in a solution to (7). Thus, we apply a greedy postprocessing procedure in order to identify high-quality solutions as early as possible in the course of the branch-and-cut. For the sake of exposition, we assume that $\bar{Z}_{\bar{R}}$ is updated to the value of such an improving solution whenever one is detected.

If $\bar{Z}_{\bar{R}} \leq \bar{Z}$, then also $\bar{Z} \leq \bar{Z}^{*}$ holds, and a feasible integer solution to the SDVRPTW-IC has been found. In case $\bar{Z}_{\bar{R}}<\bar{Z}$, the solution is a new best one, so that the best known solution value can be updated by $\bar{Z}^{*}:=\bar{Z}_{\bar{R}}$ and the branch-and-bound node can be terminated.

If $\bar{Z}_{\bar{R}}>\bar{Z}$, the current integer solution $\bar{s}$ is infeasible, and a feasibility cut must be added (see below). Moreover, the resulting branch-and-bound node must be examined further. It is worth noting that the upper bound $\bar{Z}^{*}$ can however be updated by $\bar{Z}^{*}:=\bar{Z}_{\bar{R}}$ if $\bar{Z}_{\bar{R}}<\bar{Z}^{*}$ holds.

Feasibility Cuts The definitions of valid feasibility cuts and the procedures to identify them are different depending on whether service-time flow variables $T_{i j}$ occur in the objective (i.e., $\gamma>0$ in $(3 \mathrm{a}))$ or not $(\gamma=0)$. The case of $\gamma=0$ is identical to what is described in (Bianchessi and Irnich 2018) so that we sketch it only briefly here. The case of $\gamma>0$ requires a special treatment that we describe afterwards.

If $\gamma=0$, feasibility cuts are generated as follows. Integer solutions $\bar{s}$ to $(3)$ often partition the set of customers into several weakly connected components. Defining $\mathcal{C}$ as the index set of these components, let $\bar{N}^{c}$, for each $c \in \mathcal{C}$, be the vertex set of the $c$ th weakly connected component of $H(V, \overline{\mathbf{x}})(N)$, i.e., of the vertex-induced subgraph of $H(V, \overline{\mathbf{x}})$ induced by the customers $N$. Smaller SDVRPTW-IC instances can now be defined by $\bar{V}^{c}=\bar{N}^{c} \cup\{0, n+1\}$.

For each $c \in \mathcal{C}$, we define $\bar{x}_{i j}^{c}=\bar{x}_{i j}$ if $(i, j) \in \bar{V}^{c} \times \bar{V}^{c}$, and 0 otherwise. Then, we build $H\left(\bar{V}^{c}, \overline{\mathbf{x}}^{c}\right)=$ $\left(\bar{V}^{c}, \bar{A}^{c}\right)$, generate the routes $\bar{R}$ over $H\left(\bar{V}^{c}, \overline{\mathbf{x}}^{c}\right)$, and solve the resulting formulation (7). Note that, in order to speed up the solution process, here we define $\bar{A}^{c}=\left\{(i, j) \in A \cap\left(\bar{V}^{c} \times \bar{V}^{c}\right): \bar{x}_{i j} \geq 1\right\}$ and 
impose in (7) to use each arc $(i, j) \in \bar{V}^{c} \times \bar{V}^{c}$ exactly $\bar{x}_{i j}^{c}$ times (the additional constraints are of the form $\sum_{r \in \bar{R}} b_{i j}^{r} \lambda^{r}=\bar{x}_{i j}^{c}$ ). Moreover, we set $\bar{Z}^{*}$ in $(7 \mathrm{~b})$ to $\bar{Z}^{c}:=\mathbf{c}^{\top} \overline{\mathbf{x}}^{c}$.

If $(7)$ is infeasible, we add the following feasibility cut defined w.r.t. the $c$ th weakly connected component $\bar{N}^{c}$

$$
\sum_{(i, j) \in \widehat{A}^{c}} x_{i j} \geq 1
$$

where the arc set $\widehat{A}^{c}$ defining the left-hand side is

$$
\widehat{A}^{c}=\left\{(i, j) \in A \cap\left(\bar{V}^{c} \times \bar{V}^{c}\right): \bar{x}_{i j}=0\right\} \cup \Gamma_{N}^{+}\left(\bar{N}^{c}\right) \cup \Gamma_{N}^{-}\left(\bar{N}^{c}\right) .
$$

The cut (8) imposes that either the set of active vehicle flow variables associated with the internal arcs of component $c$ must be different from the ones positive in the solution $\bar{s}$ or the component $c$ itself must change. The inequality is globally valid. Thus, whenever $\bar{s}$ has been proved to be infeasible for the SDVRPTW-IC, it can be cut off by imposing to change the current solution for at least one connected component of $H(V, \overline{\mathbf{x}})$. It happens regularly that lifted feasibility cuts for several components can be added at the same time.

If $\gamma>0$, i.e., if the objective contains costs related to route durations, the checking procedure outlined above is not directly applicable, as it may erroneously prevent a component $\bar{N}^{c}$ from being part of a solution. This is caused by the combined effect of the following: (i) the solution of the relaxed model (3) may underestimate the costs of a component (see Example 2 and Figure 3) and (ii) the feasibility cuts (8) are defined just in terms of the $x_{i j}$ variables, which are associated with the variable routing costs only. Thus, if $\gamma>0$, (7b) must be removed from (7) when checking the feasibility of a component. Then, a component can be proved to be infeasible due to the violation of vehicle capacity, time-window, or synchronization constraints, so that a feasibility cut (8) can be added for this component. The remaining inconvenience constraints are always satisfied, because we impose the additional constraints $\sum_{r \in \bar{R}} b_{i j}^{r} \lambda^{r}=\bar{x}_{i j}^{c}$ for all $(i, j) \in \bar{A}^{c}$ when checking the feasibility of a component. If none of the components is infeasible, the feasibility cut for checking the whole solution has to be added to the model, i.e., the feasibility cut defined for the arc set $\widehat{A}^{c}=\left\{(i, j) \in A: \bar{x}_{i j}=0\right\}$.

\section{Experimental Results}

The branch-and-cut algorithm was implemented in C++ using CPLEX 12.6.0.1 with Concert Technology, and compiled in release mode with MS Visual C++ 2013. The experiments were performed on a 64-bit Windows 10 PC equipped with an Intel Xeon processor E5-1650v3 clocked at $3.50 \mathrm{GHz}$ and with 64 GB of RAM, by allowing a single thread for each run. CPLEX's built-in cuts were used in all experiments. To improve numerical stability, we set IloCplex: :NumericalEmphasis 
Bianchessi, Drexl, and Irnich: The SDVRPTW and Customer Inconvenience Constraints

Article submitted to Transportation Science; manuscript no. TS-2017-0288.R2

$=$ CPX_ON and IloCplex::EpGap equal to $1.0 \mathrm{e}-5$ for fixed vehicle costs $C=0$ and to $1.0 \mathrm{e}-9$ for $C=1,000,000$ respectively. Finally, we set IloCplex : ParallelMode $=1$ in order to force CPLEX to always use deterministic algorithms. CPLEX's default values were kept for all remaining parameters.

\subsection{Instances}

In Section 1, we found that the standard benchmark for SDVRPTW, which is based on the wellknown VRPTW instances by Solomon (1987), lacks generality because instances do not exhibit different demand distributions. The demand distribution, however, strongly impacts the average savings resulting from allowing split deliveries. Therefore, we created 560 new test instances, again derived from the instances by Solomon (1987). Recall that the Solomon instance set comprises 56 instances, each of which contains 100 customers located in a $100 \times 100$ square. The set is divided into 6 classes termed $\mathrm{R} 1, \mathrm{R} 2, \mathrm{C} 1, \mathrm{C} 2, \mathrm{RC} 1$, and $\mathrm{RC} 2$, where "R" stands for "random", "C" for "clustered", and "RC" for "random and clustered", thus denoting the manner in which the customers are located in the square. The "2" instances have less constraining time windows and larger vehicle capacities than the "1" instances, so that longer routes are possible. Costs and travel times between customers are set to the Euclidean distance, customer demands are integer, and the vehicles are assumed to be homogeneous. Each class contains between 8 and 12 instances.

For the new instances, the vehicle capacity $Q$ is set to 100 . We consider five scenarios with regard to the customer demands:

$$
D 1:[10 ; 70] \quad D 2:[10 ; 50] \quad D 3:[30 ; 70] \quad D 4:[30 ; 50] \quad D 5:[50 ; 70]
$$

In each of the five scenarios $[a, b]$, the demand $d_{i}$ of customers $i \in N$ is drawn from a discrete uniform distribution in $\left[\frac{a}{100} Q, \frac{b}{100} Q\right]$. As in the original Solomon benchmark, all instances of a class (e.g., R1) share the identical demand realization in a scenario.

From each instance, we derived 25- and 50-customer instances by considering only the first 25 and 50 customers respectively. Hence, we obtained $56 \cdot 5 \cdot 2=560$ instances, available at http: //logistik.bwl.uni-mainz.de/benchmarks.php. We partitioned the instances into groups by Solomon class, demand scenario, and number of customers. For example, "C1D2N25" refers to the 25-customer instances created from Solomon class C1 with demands in $[10 ; 50]$.

By convention, we computed travel times and variable routing costs with one decimal place and truncation. Then, as the triangle inequality is assumed to hold for both times and costs, at preprocessing time we apply the Floyd-Warshall algorithm to times and costs independently. Hence, the new instances allow us to require all Properties 1-5 for optimal solutions. 


\subsection{Results}

We considered the eight distribution policies described in Table 2. The extreme policies are those leading to the VRPTW (no splitting at all) and the SDVRPTW (arbitrary splits allowed), while the introduction of the inconvenience measures creates variants of the SDVRPTW-IC.

Table 2 The different distribution policies considered in the computational experiments

\begin{tabular}{ll}
\hline Policy & Meaning \\
\hline VRPTW & Standard VRP with time windows. \\
SDVRPTW & Split delivery VRP with time windows. \\
$\mathrm{S} \Delta, \quad$ for $\Delta=0$ & SDVRPTW with temporal synchronization of deliveries/visits. $\Delta=0$ is \\
& exact temporal synchronization. \\
$\mathrm{NV} \nu$, for $\nu=2,3$ & SDVRPTW with at most $\nu$ visits per customer, i.e., $n_{i}^{\max }=\nu$ for all \\
& customers $i \in N$. \\
TNV $x$, & SDVRPTW with a limit on the total number of visits, $n^{\text {max } . ~ F o r ~ a n ~}$ \\
for $x=25,50,75$ & instance with $n$ customers and $\xi$ visits in the optimal SDVRPTW solu- \\
& tion, $n^{\max }=n+\left\lceil\frac{x}{100} \cdot(\xi-n)\right\rceil$. \\
& Example: For an instance with $n=50$ for which the optimal SDVRPTW \\
& solution visits ten customers twice and no customer more than twice, \\
& $\xi=60$, and for $x=25, n^{\max }=53$. \\
&
\end{tabular}

The VRPTW served as baseline against which the other distribution policies were compared, except for the results in Section 5.2.2, for which the SDVRPTW was used as baseline. We consider synchronization and limiting the number of visits as alternative measures for controlling inconvenience and therefore analyzed them separately; mixing them makes no sense in our opinion.

We performed three sets of experiments using different objectives (henceforth referred to as objective I, II, and III), as defined in Table 3.

Table 3 The different objective functions used in the computational experiments

\begin{tabular}{lccc}
\hline & \multicolumn{3}{c}{ Objective function components } \\
\cline { 2 - 4 } $\begin{array}{l}\text { Objective } \\
\text { function }\end{array}$ & $\begin{array}{c}\text { Variable } \\
\text { routing costs }\end{array}$ & $\begin{array}{c}\text { Costs related to } \\
\text { route durations }\end{array}$ & $\begin{array}{c}\text { Fixed } \\
\text { vehicle costs }\end{array}$ \\
\hline I & yes & no & no \\
II & yes & yes: $\gamma=1$ & no \\
III & yes & yes: $\gamma=1$ & yes: $C=1,000,000$ \\
\hline
\end{tabular}

In the first set, we used the minimization of total variable routing costs. We analyzed the structure of the different solutions comparing the objective function values and the impact of the distribution policies on route durations and on the number of routes. In the second set, we included the costs related to route durations into the objective, and in the third set, we chose a hierarchical objective 
of minimizing the number of vehicles first (by setting very high fixed vehicle costs) and minimizing the sum of variable routing costs and costs related to route durations second.

Except for the results presented in Section 5.2.2, an instance was used for the analyses only when it had been solved to optimality for all policies (apart from NV3, as only very few instances had more than three visits in the optimal SDVRPTW solution) and all objective functions. This was the case for 115 instances, 109 of which had 25 customers. The results discussed in Section 5.2.2 were obtained using, for each objective, the instances that were solved to optimality with the SDVRPTW policy for this objective. This is because the TNVx policies can, by definition, be applied to these instances only.

\subsection{1. $\quad$ Effect of the Different Objective Functions and Distribution Policies Table 4} contains structural information about the effect of allowing split deliveries according to the different objective functions. It displays several indicators that quantify how the optimal solutions of the policies with splits differ from those of the respective VRPTW. The last column deserves some explanation. If, for example, a customer with a time window of $[10,20]$ is visited twice, at time points 13 and 16, then the "timespan between the first and the last delivery in relation to time window width" is $(16-13) /(20-10)=0.3=30 \%$. Note that the values in this column are based on the original time windows (as these would be given by the customers), not on the ones reduced according to the minimum arrival time from the depot and the maximum departure time to reach the depot.

Table 5 provides information on the benefits of split deliveries. The table shows the minimum, average, and maximum relative savings in $\%$ and the number of instances with savings of more than $3 \%$ for the different objective functions, each compared to the VRPTW policy with the respective objective. Note: It turned out that there are only very few instances with more than two visits to any customer, so the results for policy NV3 are omitted from the analyses.

5.2.1.1. Comparison of VRPTW and SDVRPTW Looking at Table 4, one can see that the percentage of split customers depends strongly on the objective function. This also holds for the percentage of split customers for which the deliveries are fully synchronized automatically, i.e., for which all deliveries occur at the same time without requiring this by a constraint. Both values are by far highest for objective I, i.e., when only variable routing costs are taken into account.

Table 5 shows that for objective I, i.e., the minimization of variable routing costs, considerable savings in the objective value and in the number of routes are realized, averaging to 2.6 and $2.3 \%$ respectively, with reductions of more than $3 \%$ for 47 and 30 instances out of 115 . Route durations, however, show a large average increase of $7.9 \%$. What is more, the volatility of the route duration changes is high, ranging from a duration reduction of $17.0 \%$ to an increase of as much as $81.5 \%$. 
Table 4 Effect of the different objective functions and distribution policies on solution structure compared to

\begin{tabular}{|c|c|c|c|c|c|}
\hline \multirow[b]{3}{*}{$\begin{array}{c}\text { Objective/ } \\
\text { Policy }\end{array}$} & \multicolumn{5}{|c|}{ VRPTW } \\
\hline & \multicolumn{5}{|c|}{ Average of } \\
\hline & $\begin{array}{l}\text { Number of } \\
\text { visits per } \\
\text { customer }\end{array}$ & $\begin{array}{l}\text { Percentage } \\
\text { of split } \\
\text { customers }\end{array}$ & $\begin{array}{l}\text { Number of } \\
\text { visits per } \\
\text { split } \\
\text { customer }\end{array}$ & $\begin{array}{c}\text { Percentage of } \\
\text { split customers } \\
\text { with deliveries } \\
\text { fully } \\
\text { synchronized }\end{array}$ & $\begin{array}{l}\text { Timespan between } \\
\text { first and last } \\
\text { delivery in relation } \\
\text { to time window } \\
\text { width in \% }\end{array}$ \\
\hline \multicolumn{6}{|l|}{ Objective I } \\
\hline SDVRPTW & 1.10 & 9.84 & 2.00 & 20.95 & 29.44 \\
\hline NV2 & 1.10 & 9.98 & 2.00 & 21.37 & 28.37 \\
\hline So & 1.10 & 9.86 & 2.00 & 100.00 & 0.00 \\
\hline TNV25 & 1.03 & 3.06 & 2.00 & 22.09 & 24.11 \\
\hline TNV50 & 1.06 & 5.53 & 2.02 & 21.88 & 30.60 \\
\hline TNV75 & 1.08 & 7.84 & 2.00 & 21.35 & 28.17 \\
\hline \multicolumn{6}{|l|}{ Objective II } \\
\hline SDVRPTW & 1.03 & 2.99 & 2.01 & 10.00 & 13.76 \\
\hline NV2 & 1.03 & 2.99 & 2.00 & 10.74 & 14.21 \\
\hline So & 1.02 & 2.40 & 2.01 & 100.00 & 0.00 \\
\hline TNV25 & 1.01 & 0.89 & 2.00 & 15.38 & 8.95 \\
\hline TNV50 & 1.02 & 1.65 & 2.00 & 10.98 & 12.86 \\
\hline TNV75 & 1.02 & 2.37 & 2.00 & 10.00 & 12.85 \\
\hline \multicolumn{6}{|l|}{ Objective III } \\
\hline SDVRPTW & 1.04 & 3.72 & 2.01 & 19.17 & 17.18 \\
\hline NV2 & 1.04 & 3.72 & 2.00 & 11.39 & 19.25 \\
\hline So & 1.03 & 3.10 & 2.01 & 100.00 & 0.00 \\
\hline TNV25 & 1.01 & 1.13 & 2.00 & 15.15 & 8.92 \\
\hline TNV50 & 1.03 & 2.56 & 2.00 & 18.33 & 17.71 \\
\hline TNV75 & 1.03 & 3.20 & 2.00 & 15.00 & 17.32 \\
\hline
\end{tabular}

As a side effect, assuming $\gamma=1$ as for the other objectives, the sum of variable routing costs and costs related to route durations increases on average by about $5.9 \%$. In particular, increases occur also when the number of vehicles is not reduced.

The picture changes for objective II, i.e., when variable routing costs and costs related to route durations are minimized simultaneously. Then, the average savings in the objective function as well as in the number of routes, although still non-negligible, are much lower than for objective I, and there is no instance with an objective reduction of more than $3 \%$. This indicates that split deliveries pay off less when variable routing as well as duration-related costs are considered compared to the situation where only variable routing costs matter. Route durations and variable routing costs are hardly affected, and their volatility is small, with percentage savings ranging in $[-2.3,2.0]$ and $[-1.2,4.9]$ respectively.

For objective III, i.e., the hierarchical objective of minimizing first the number of routes and then the sum of variable routing costs and costs related to route durations, we observe that there is only a marginal reduction in the number of routes. For the sum of variable routing costs and costs related to route durations, however, substantial savings are obtained, of $2.2 \%$ on average, and with a maximum of $36.7 \%$. (Note that increases in the second objective function component occurred, but only when the number of vehicles was reduced.) The volatilities of the changes for variable routing costs and route durations are relevant and even higher than those found for objective I. 
Bianchessi, Drexl, and Irnich: The SDVRPTW and Customer Inconvenience Constraints

Table 5 Relative savings obtained with the different objective functions and distribution policies compared to VRPTW

\begin{tabular}{|c|c|c|c|c|c|}
\hline \multirow[b]{2}{*}{$\begin{array}{r}\text { Objective/ } \\
\text { Policy }\end{array}$} & \multicolumn{5}{|c|}{ Min./Avg./Max. \% Savings/\# Instances with savings $>3 \%$ in } \\
\hline & Objective value & Number of routes & $\begin{array}{c}\text { Variable routing } \\
\text { costs }\end{array}$ & Route durations & $\begin{array}{l}\text { Sum of variable } \\
\text { routing costs and } \\
\text { costs related to } \\
\text { route durations }\end{array}$ \\
\hline \multicolumn{6}{|l|}{ Objective I } \\
\hline SDVRPTW & $0.00 / 2.55 / 8.87 / 47$ & $0.00 / 2.25 / 13.33 / 30$ & $0.00 / 2.55 / 8.87 / 47$ & $-81.47 /-7.91 / 16.99 / 8$ & $-70.56 /-5.87 / 15.84 / 8$ \\
\hline NV2 & $0.00 / 2.55 / 8.87 / 47$ & $0.00 / 2.25 / 13.33 / 30$ & $0.00 / 2.55 / 8.87 / 47$ & $-81.47 /-7.92 / 18.46 / 9$ & $-70.56 /-5.87 / 17.19 / 9$ \\
\hline So & $0.00 / 2.50 / 8.87 / 42$ & $0.00 / 2.25 / 13.33 / 30$ & $0.00 / 2.50 / 8.87 / 42$ & $-402.52 /-54.36 / 2.31 / 0$ & $-332.27 /-40.32 / 2.39 / 0$ \\
\hline TNV25 & $0.00 / 1.30 / 5.49 / 16$ & $-10.00 / 0.00 / 10.00 / 11$ & $0.00 / 1.30 / 5.49 / 16$ & $-46.82 /-3.42 / 27.69 / 13$ & $-38.40 /-2.65 / 25.14 / 11$ \\
\hline TNV50 & $0.00 / 2.15 / 8.19 / 25$ & $0.00 / 2.11 / 13.33 / 28$ & $0.00 / 2.15 / 8.19 / 25$ & $-42.10 /-4.81 / 34.26 / 12$ & $-35.64 /-3.62 / 24.15 / 10$ \\
\hline TNV75 & $0.00 / 2.42 / 8.45 / 40$ & $0.00 / 2.19 / 13.33 / 29$ & $0.00 / 2.42 / 8.45 / 40$ & $-56.30 /-6.46 / 34.26 / 12$ & $-47.81 /-4.82 / 24.15 / 11$ \\
\hline \multicolumn{6}{|l|}{ Objective II } \\
\hline SDVRPTW & $0.00 / 0.47 / 2.07 / 0$ & $0.00 / 1.17 / 18.18 / 15$ & $-1.15 / 1.03 / 4.86 / 21$ & $-2.34 / 0.06 / 2.01 / 0$ & $0.00 / 0.47 / 2.07 / 0$ \\
\hline NV2 & $0.00 / 0.47 / 2.07 / 0$ & $0.00 / 1.17 / 18.18 / 15$ & $-1.15 / 1.02 / 4.86 / 21$ & $-2.34 / 0.06 / 2.01 / 0$ & $0.00 / 0.47 / 2.07 / 0$ \\
\hline So & $0.00 / 0.40 / 2.06 / 0$ & $-10.00 / 1.00 / 18.18 / 15$ & $0.00 / 0.91 / 4.86 / 17$ & $-2.85 / 0.03 / 1.72 / 0$ & $0.00 / 0.40 / 2.06 / 0$ \\
\hline TNV25 & $0.00 / 0.21 / 1.71 / 0$ & $-10.00 /-0.28 / 10.00 / 4$ & $0.00 / 0.37 / 2.82 / 0$ & $-0.97 / 0.09 / 1.31 / 0$ & $0.00 / 0.21 / 1.71 / 0$ \\
\hline TNV50 & $0.00 / 0.32 / 2.01 / 0$ & $-10.00 / 0.43 / 10.00 / 8$ & $-1.15 / 0.60 / 3.14 / 1$ & $-1.66 / 0.11 / 2.01 / 0$ & $0.00 / 0.32 / 2.01 / 0$ \\
\hline TNV75 & $0.00 / 0.41 / 2.01 / 0$ & $-10.00 / 0.76 / 18.18 / 12$ & $-1.15 / 0.84 / 4.86 / 9$ & $-2.34 / 0.10 / 2.01 / 0$ & $0.00 / 0.41 / 2.01 / 0$ \\
\hline \multicolumn{6}{|l|}{ Objective III } \\
\hline SDVRPTW & $0.00 / 0.09 / 9.07 / 1$ & $0.00 / 0.08 / 9.09 / 1$ & $-2.50 / 2.43 / 12.71 / 37$ & $-2.51 / 1.77 / 40.57 / 15$ & $-2.50 / 2.15 / 36.72 / 17$ \\
\hline NV2 & $0.00 / 0.09 / 9.07 / 1$ & $0.00 / 0.08 / 9.09 / 1$ & $-2.50 / 2.43 / 12.71 / 37$ & $-2.51 / 1.78 / 40.57 / 15$ & $-2.50 / 2.15 / 36.72 / 17$ \\
\hline So & $0.00 / 0.09 / 9.07 / 1$ & $0.00 / 0.08 / 9.09 / 1$ & $-2.50 / 2.31 / 12.71 / 34$ & $-3.39 / 1.69 / 40.17 / 15$ & $-3.03 / 2.04 / 36.41 / 16$ \\
\hline TNV25 & $0.00 / 0.08 / 9.07 / 1$ & $0.00 / 0.08 / 9.09 / 1$ & $-4.15 / 0.76 / 12.30 / 8$ & $-2.41 / 0.74 / 37.93 / 8$ & $-3.11 / 0.83 / 34.43 / 8$ \\
\hline TNV50 & $0.00 / 0.09 / 9.07 / 1$ & $0.00 / 0.08 / 9.09 / 1$ & $-4.15 / 2.01 / 13.72 / 19$ & $-2.41 / 1.65 / 39.08 / 15$ & $-3.11 / 1.89 / 35.61 / 16$ \\
\hline TNV75 & $0.00 / 0.09 / 9.07 / 1$ & $0.00 / 0.08 / 9.09 / 1$ & $-2.50 / 2.29 / 13.72 / 26$ & $-2.51 / 1.77 / 39.08 / 15$ & $-2.50 / 2.08 / 35.61 / 17$ \\
\hline
\end{tabular}

However, percentage savings ranges are now unbalanced towards positive values. For 17 out of 115 instances, the value of at least one of the two objective function components was reduced by at least 3\%. In conclusion, it can be said that splitting pays off for objective III, and more so than for objective II.

5.2.1.2. Comparison of the Distribution Policies for the Reduction of Inconvenience Having established the usefulness of split deliveries empirically, we evaluate in this section the different measures for reducing inconvenience that may result from splitting.

Table 4 shows that the relative values of the structural indicators within one objective function are similar for all three of them: (i) The percentage of split customers is lower when there is a limit on the total number of visits. (ii) The percentage of fully synchronized visits and the average time span between the first and the last delivery per split customer in relation to the time window width are similar for all policies without explicit synchronization. In particular, the latter value is rather high, which may be regarded a considerable inconvenience for customers.

Looking at Table 5, the most striking observations are: (i) A limit on the overall number of visits yields, in general, smaller objective function reductions than the other measures. (ii) The NV-2 
values for all columns are almost the same as for the corresponding SDVRPTW. (iii) Most notably, when costs related to route durations are ignored in the objective function, their increase is drastic for the synchronized SDVRPTW, with an average of $54.4 \%$ and a maximum of $402.5 \%$. However, when costs related to route durations are taken into account, the duration differences between the SDVRPTW and the S0 policies are minimal. (iv) Objective function values of the SDVRPTW and the S0 policies differ only slightly for all three objectives.

As a limit on the number of individual visits does not improve the quality of service to the customers, synchronization, i.e., the S0 distribution policy, can be seen as the best measure to mitigate the customer inconvenience, leading to a win-win situation for carriers and customers.

5.2.1.3. In-Depth Analysis of Objective II Objective II is important because it is the one that balances the two most critical and conflicting cost components: it simultaneously minimizes variable routing costs and costs related to route durations. In order to further validate and extend the findings stated in Sections 5.2.1.1 and 5.2.1.2, we carried out an in-depth analysis of objective II.

Limiting the scope to objective II, 205 instances were solved to optimality with all policies, including 18 instances with 50 customers. We obtained identical optimal SDVRPTW and VRPTW solutions for 112 of these 205 instances (identical w.r.t. to the objective function value and the number of vehicles used). In Figure 4, we display, for the remaining 93 instances and the different distribution policies, the savings achieved in total costs and number of vehicles. Information is grouped by demand scenario.

Even if cost savings are on average smaller than for objective I as stated in Section 5.2.1.1, allowing split deliveries for objective II is still a worthwhile alternative. Indeed, the magnitude of the savings very much depends on the demand distribution. Figure 4(c) reveals that, for many instances, substantial savings can be achieved, in particular in demand scenario D3.

As for the comparison of the distribution policies, the difference between NV2 and SDVRPTW is marginal:

- NV2 achieves the same cost savings as SDVRPTW in all but two cases.

- The number of vehicles used is identical for NV2 and SDVRPTW.

- NV2 is as inconvenient for customers as SDVRPTW; it reduces the number of visits only in rare cases.

Regarding cost savings w.r.t. VRPTW, the difference in savings achieved between NV2 and S0 is greater than $0.5 \%$ (1\%) in only 13 (2) out of 205 cases, with a maximum of $1.26 \%$. Then, comparing the optimal solutions, we found that

- in 9 out of 205 cases, S0 uses 1 vehicle more than for NV2;

- in 22 (1) out of 205 cases, TNV75 uses 1 (2) vehicle(s) more than NV2;

- in $31(11,1)$ out of 205 cases, TNV50 uses $1(2,3)$ vehicle(s) more than NV2; 
- in $43(18,1)$ out of 205 cases, TNV25 uses $1(2,5)$ vehicle(s) more than NV2.

Thus, as observed in Section 5.2.1.2, synchronization with policy S0 is, w.r.t. total costs, the third best option after SDVRPTW and NV2. Nevertheless, S0 is superior to SDVRPTW and NV2 in reducing customer inconvenience, because in the former all visits to a customer occur at the same time. 


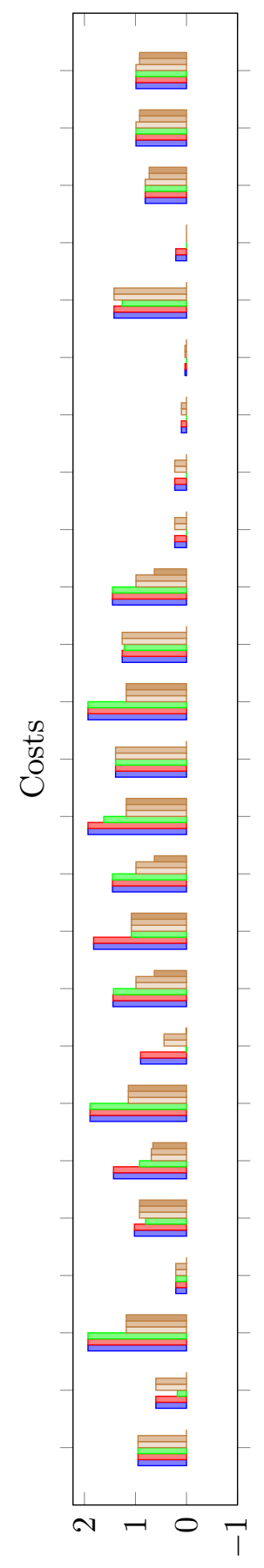

(\%) 8u!̣es

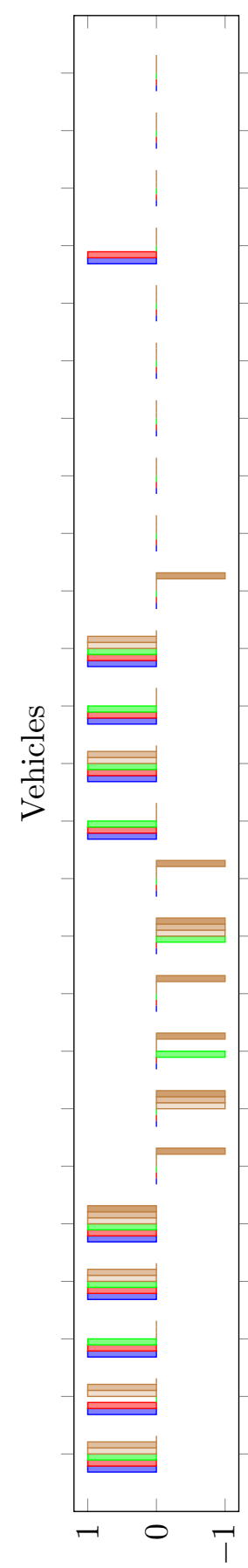

8ันเ้̣อง

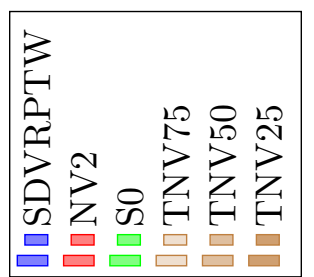

sis

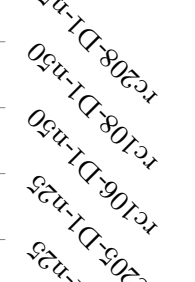

वर्दूय

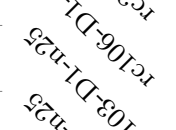

$a^{2} a^{a^{2} \partial^{2}}$

वृद्ये

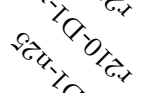

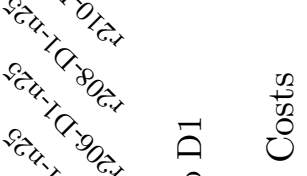

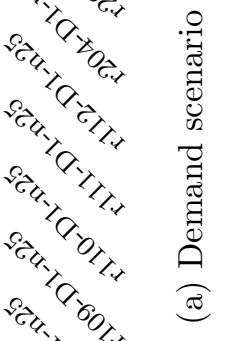

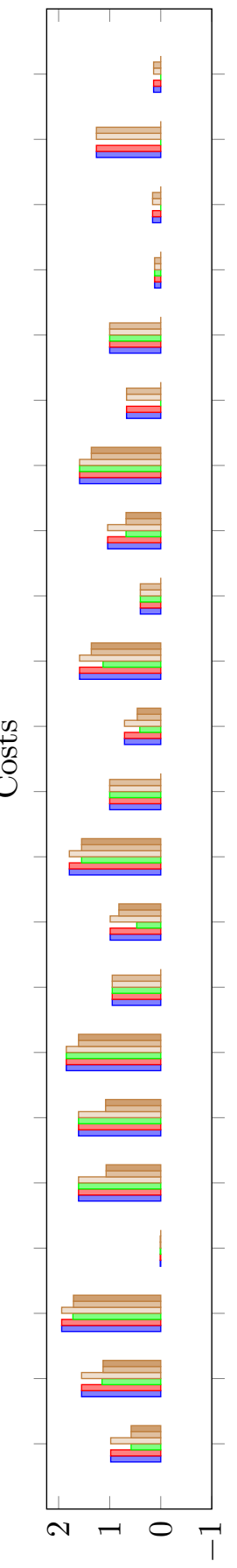

(\%) 8u!nes

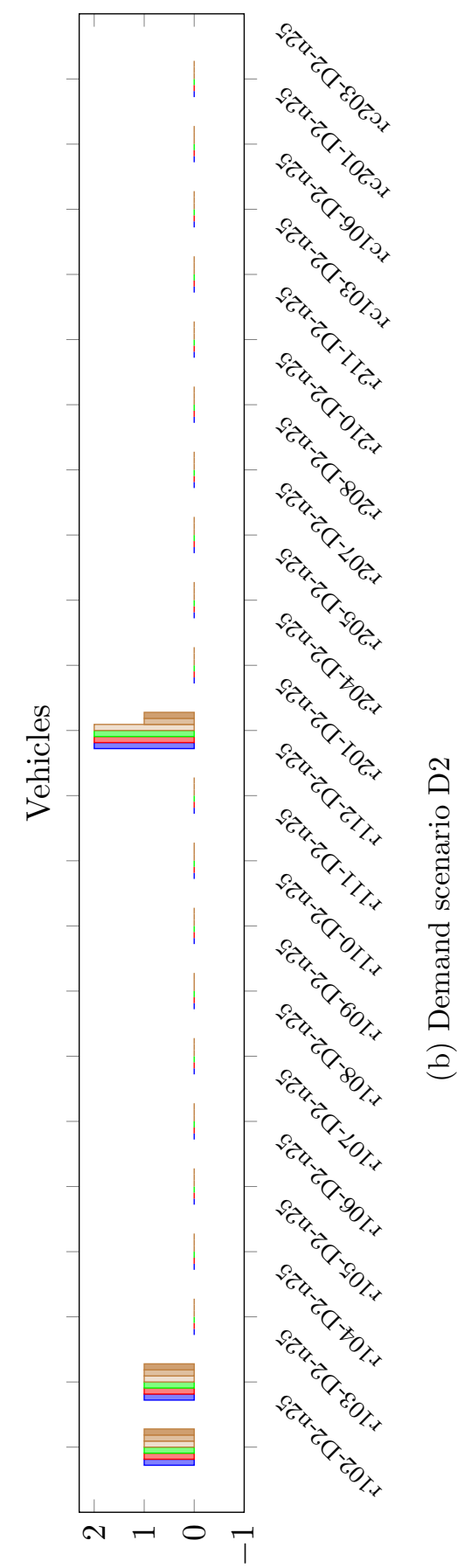

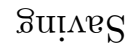




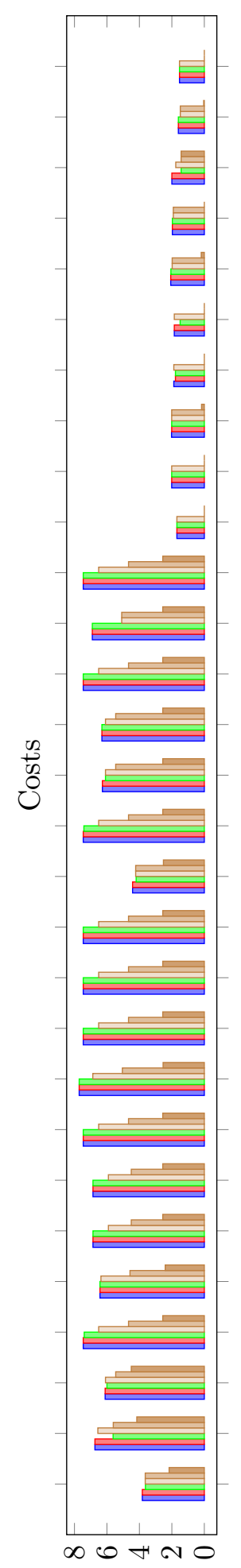

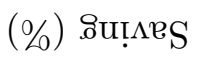
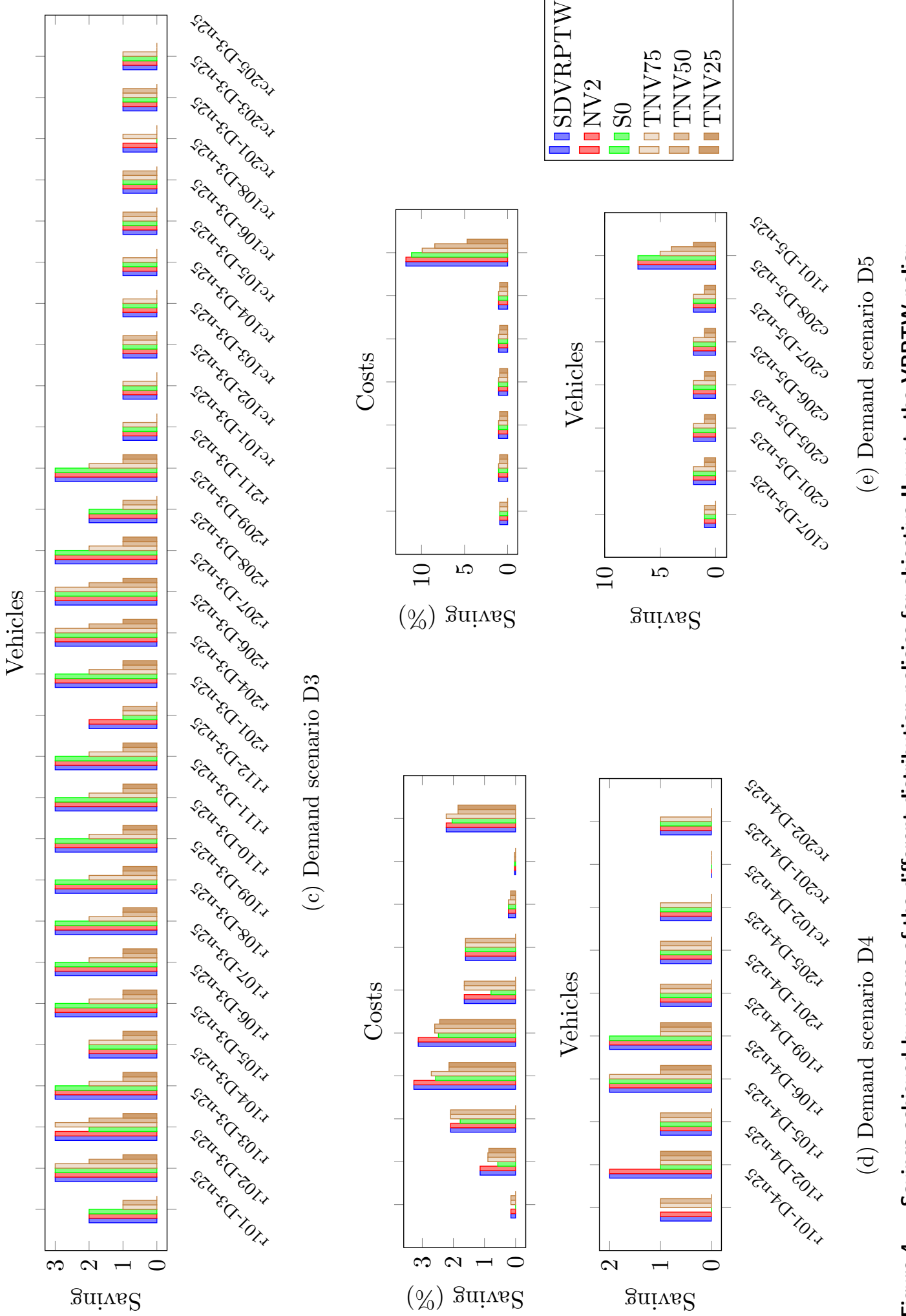

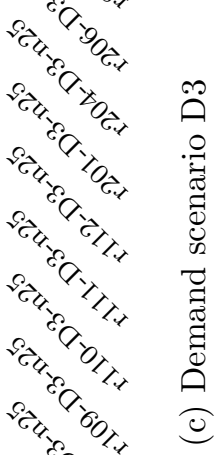
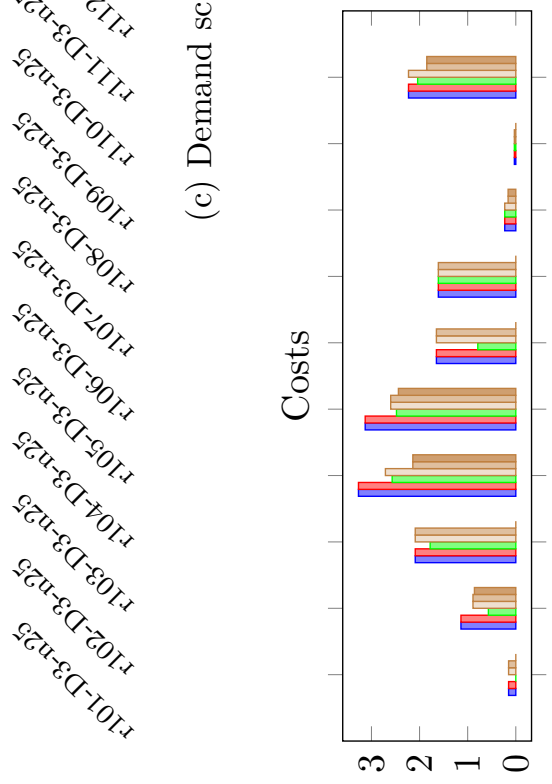

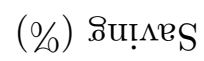
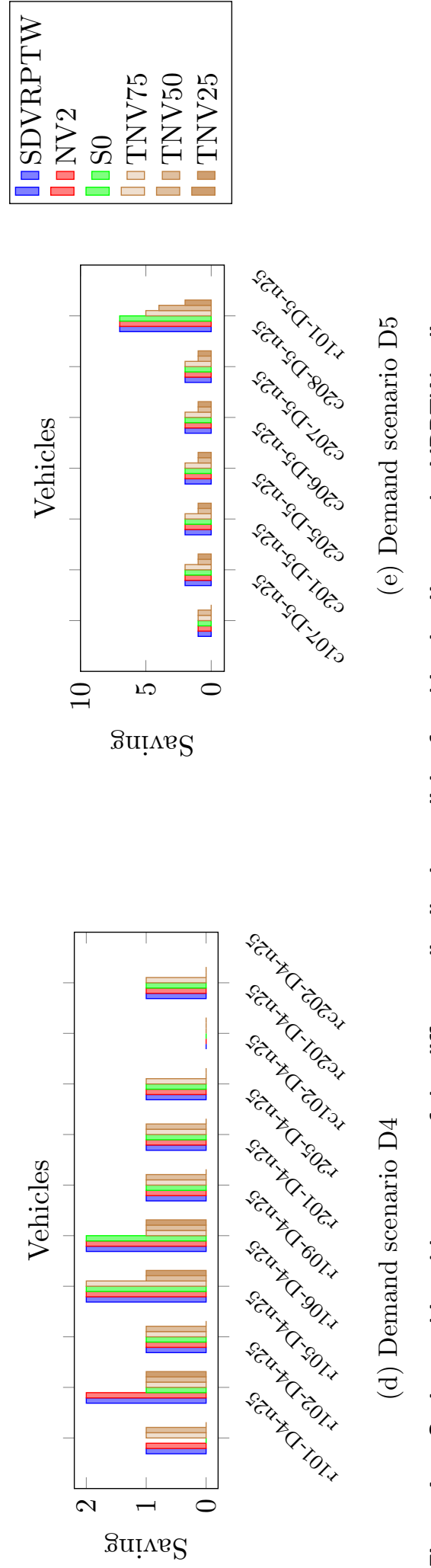


\subsubsection{Impact of Inconvenience Constraints on the Performance of the Branch-} and-Cut Table 6 presents information on how the different policies affect the performance of our algorithm. Recall that the results for this subsection were obtained using, for each objective, the instances that were solved to optimality with the SDVRPTW policy for this objective. The first column of the table presents the number of optimal solutions computed within one hour of CPU time. The subsequent columns show the arithmetic means of (i) the running times, (ii) the number of branch-and-bound nodes, (iii) the numbers of cuts of the different types, (iv) the percentage of the total time spent for separating the cuts used to strengthen the formulation, and (v) the percentage of the total time spent for separating the feasibility cuts. (As the cuts for strengthening the formulation were separated in a hierarchical manner as described in Section 4.2, it would make no sense to provide one column for the relative separation time of each such cut type.) It can be seen from the table that, in general, adding inconvenience constraints to the SDVRPTW makes the problem harder: the number of optimal solutions is much lower for all other policies except NV2, whereas the running times as well the number of branch-and-bound nodes generally increase. The "easiest" type of inconvenience constraints is NV2, i.e., limiting the number of visits to two, with the highest number of optimal solutions and the lowest average running times. With regard to objective functions, judging from the number of optimal solutions and, to a lesser extent, the average running times, objective III is the most difficult.

The number of separated cuts is not monotonous: although further down the separation hierarchy, much more infeasible-path cuts and path-matching cuts were separated than 2-path and connectivity cuts, except for the NV2 policy. For the TNVx policies, the number of separated capacity cuts is significantly higher than for the other policies. On the other hand, the number of feasibility cuts is clearly highest for policy S0 and comparably low for the TNVx policies. This implies that integer feasibility is harder to attain for S0.

Relative separation times for the TNVx policies are a bit lower for the formulation-strengthening cuts and much lower for the feasibility cuts than for the other policies. For both types of cuts, the average relative separation times are generally below $8 \%$, i.e., for most instances, solving the LP relaxation is the time-consuming part. For all objectives, it can be seen from the penultimate column of Table 6 that, with one exception, the relative time needed to separate the formulationstrengthening cuts is lower compared to the baseline SDVRPTW policy.

Further analyses, not presented in the table, showed that the volatility of relative separation times of feasibility cuts is extremely high. Even within the same demand scenario, these proportions range from 0 to almost 100\%, i.e., for a few instances, almost the complete running time was spent for separating feasibility cuts. For the cuts for strengthening the formulation, this volatility is much less pronounced, with proportions ranging from 0 to at most $35 \%$, but again, this range appears within the same scenario. 
Bianchessi, Drexl, and Irnich: The SDVRPTW and Customer Inconvenience Constraints

Table 6 Impact of inconvenience constraints on algorithm performance

\begin{tabular}{|c|c|c|c|c|c|c|c|c|c|c|c|}
\hline \multirow[b]{2}{*}{$\begin{array}{r}\text { Objective/ } \\
\text { Policy }\end{array}$} & \multirow[b]{2}{*}{$\begin{array}{c}\text { Num. } \\
\text { opt. } \\
\text { sols. }\end{array}$} & \multicolumn{10}{|c|}{ Average of } \\
\hline & & $\begin{array}{l}\text { Run } \\
\text { time }\end{array}$ & $\begin{array}{l}\text { B\&B } \\
\text { nodes }\end{array}$ & $\begin{array}{l}\text { Cap. } \\
\text { cuts }\end{array}$ & $\begin{array}{l}\text { 2-path } \\
\text { cuts }\end{array}$ & $\begin{array}{l}\text { Conn. } \\
\text { cuts }\end{array}$ & $\begin{array}{c}\text { Inf.- } \\
\text { path. } \\
\text { cuts }\end{array}$ & $\begin{array}{c}\text { Path- } \\
\text { match. } \\
\text { cuts }\end{array}$ & $\begin{array}{l}\text { Feas. } \\
\text { cuts }\end{array}$ & $\begin{array}{l}\text { Relativ } \\
\text { separation } \\
\text { strength. } \\
\text { cuts }\end{array}$ & $\begin{array}{l}\text { time } \\
\text { feas. } \\
\text { cuts }\end{array}$ \\
\hline \multicolumn{12}{|l|}{ Objective I } \\
\hline SDVRPTW & 396 & 131 & 2,681 & 1,417 & 0.5 & 0.5 & 24.4 & 9.0 & 77.5 & 7.67 & 3.87 \\
\hline NV2 & 395 & 118 & 2,378 & 1,425 & 0.4 & 0.4 & 19.0 & 6.0 & 38.8 & 7.93 & 2.25 \\
\hline So & 387 & 202 & 3,826 & 1,471 & 0.6 & 0.6 & 28.1 & 10.7 & 112.6 & 7.39 & 5.61 \\
\hline TNV25 & 261 & 1,455 & 10,755 & 8,022 & 1.4 & 0.1 & 35.8 & 0.7 & 0.0 & 4.97 & 0.07 \\
\hline TNV50 & 284 & 1,130 & 11,172 & 5,774 & 1.2 & 0.1 & 42.4 & 2.5 & 0.2 & 5.98 & 0.17 \\
\hline TNV75 & 336 & 678 & 15,888 & 3,151 & 0.7 & 0.1 & 32.8 & 3.3 & 1.0 & 6.75 & 0.30 \\
\hline \multicolumn{12}{|l|}{ Objective II } \\
\hline SDVRPTW & 287 & 266 & 7,384 & 1,951 & 2.0 & 0.4 & 21.5 & 6.7 & 55.3 & 6.88 & 0.85 \\
\hline NV2 & 281 & 339 & 8,932 & 2,067 & 2.0 & 0.3 & 18.8 & 5.2 & 63.5 & 6.69 & 0.89 \\
\hline So & 231 & 937 & 16,408 & 2,370 & 2.5 & 0.6 & 38.2 & 19.8 & 555.7 & 5.32 & 1.22 \\
\hline TNV25 & 235 & 878 & 9,108 & 4,829 & 2.7 & 0.0 & 23.5 & 1.5 & 8.2 & 5.07 & 0.24 \\
\hline TNV50 & 242 & 791 & 11,432 & 3,861 & 2.6 & 0.1 & 28.1 & 3.0 & 13.2 & 5.59 & 0.40 \\
\hline TNV75 & 256 & 673 & 15,843 & 2,850 & 2.4 & 0.1 & 26.6 & 4.1 & 8.7 & 5.93 & 0.43 \\
\hline \multicolumn{12}{|l|}{ Objective III } \\
\hline SDVRPTW & 254 & 351 & 9,688 & 2,100 & 2.2 & 0.4 & 32.0 & 14.5 & 90.3 & 6.89 & 4.33 \\
\hline NV2 & 245 & 442 & 11,067 & 2,355 & 2.1 & 0.3 & 27.5 & 10.3 & 90.8 & 6.81 & 2.83 \\
\hline So & 187 & 1,237 & 20,683 & 2,664 & 2.8 & 1.1 & 73.8 & 46.3 & 604.8 & 5.06 & 6.25 \\
\hline TNV25 & 145 & 1,722 & 10,486 & 10,666 & 3.8 & 0.0 & 34.8 & 3.3 & 2.1 & 3.90 & 0.14 \\
\hline TNV50 & 156 & 1,573 & 10,978 & 10,432 & 3.9 & 0.1 & 49.6 & 7.4 & 24.1 & 4.35 & 0.16 \\
\hline TNV75 & 183 & 1,229 & 14,114 & 6,791 & 3.3 & 0.2 & 45.0 & 9.4 & 18.5 & 4.95 & 0.27 \\
\hline
\end{tabular}

5.2.3. Results grouped by Demand Scenario and Solomon Class The e-Companion provides further details on the aggregated results presented in Tables 4, 5, and 6. In Section EC.2 of the e-Companion, results are grouped by demand scenario. Accordingly, Tables EC.1-EC.6 show the effects of the different objective functions on the solution structure of the various policies compared to the VRPTW, Tables EC.4-EC.6 indicate the relative savings obtained with the different objective functions, and Tables EC.7-EC.9 present the impact of the inconvenience constraints on the performance of the branch-and-cut algorithm. In Section EC.3 of the e-Companion, results are grouped by Solomon class. Tables EC.10-EC.12, EC.13-EC.15, and EC.16-EC.18 show the respective results.

\section{Conclusions}

In the present paper, we have investigated the possibilities and limitations of split deliveries with the aim of creating a win-win situation for carriers and customers in goods distribution systems. It is clear that, for the customer, it is most convenient to have only one delivery per request. However, for the carrier, split deliveries offer more degrees of freedom in routing and hence a higher optimization potential, i.e., more opportunities for cost reduction. A good trade-off between customer inconvenience and cost savings needs to be found. We focused our analysis on the vehicle routing problem with time windows in which split deliveries are allowed (SDVRPTW), and considered different distribution policies that either limit the number of visits to customers (individually and in 
total) or ensure temporally synchronized deliveries to the same customer. We evaluated the impact of these measures on carrier efficiency by means of different objective functions, each of which takes into consideration a specific combination of variable routing costs, costs related to route durations, and fixed fleet costs. The combination of these three cost components has not been considered in the literature before. We have highlighted the need to take all of them into account to provide a more complete picture of the overall logistics costs.

Based on several analyses of computational studies with a large set of instances and demand scenarios, we can make the following final recommendations to logistics managers:

- In general, split deliveries pay off; they should be considered independent of the objective.

- When variable routing costs and costs related to route durations are relevant, split deliveries are less beneficial than for other objectives, but still an alternative worth considering.

- A limit on the number of visits to individual customers is not an effective measure to mitigate customer inconvenience resulting from split deliveries, as it hardly changes the number of visits w.r.t. the SDVRPTW, i.e., it does not improve the quality of service to the customers.

- According to the average percentage of split customers, a moderate limit on the total number of visits seems to be a valid measure to reduce customer inconvenience.

- Nevertheless, the synchronization of visits allows in general to find better results. Visit synchronization, if properly implemented in practice, causes only very minor increases in any of the three components of logistics costs and therefore appears to be the most sensible and useful distribution policy.

\section{Acknowledgments}

This research was funded by the Deutsche Forschungsgemeinschaft (DFG) under grants no. IR 122/5-2 and DR 963/2-1.

\section{References}

Archetti C, Bianchessi N, Speranza MG (2011a) A column generation approach for the split delivery vehicle routing problem. Networks 58:241-254, URL http://dx.doi.org/10.1002/net.20467.

Archetti C, Bouchard M, Desaulniers G (2011b) Enhanced branch and price and cut for vehicle routing with split deliveries and time windows. Transportation Science 45(3):285-298, URL http://dx.doi.org/ $10.1287 / \operatorname{trsc} .1100 .0363$.

Archetti C, Hertz A, Speranza M (2006a) A tabu search algorithm for the split delivery vehicle routing problem. Transportation Science 40:64-73, URL http://dx.doi.org/10.1287/trsc.1040.0103.

Archetti C, Savelsbergh MWP, Speranza MG (2006b) Worst-case analysis for split delivery vehicle routing problems. Transportation Science 40(2):226-234, URL http://dx.doi.org/10.1287/trsc.1050. 0117. 
Bianchessi, Drexl, and Irnich: The SDVRPTW and Customer Inconvenience Constraints

Archetti C, Speranza MG (2012) Vehicle routing problems with split deliveries. International Transactions in Operational Research 19:3-22, URL http://dx.doi.org/10.1111/j.1475-3995.2011.00811.x.

Ascheuer N, Fischetti M, Grötschel M (2000) A polyhedral study of the asymmetric traveling salesman problem with time windows. Networks 36(2):69-79, URL http://dx.doi.org/10.1002/ 1097-0037 (200009) 36:2<69: :AID-NET1>3.0.CO;2-Q.

Ascheuer N, Fischetti M, Grötschel M (2001) Solving the asymmetric travelling salesman problem with time windows by branch-and-cut. Mathematical Programming 90(3):475-506, URL http://dx.doi.org/ $10.1007 / \mathrm{s} 101070100218$.

Bianchessi N, Irnich S (2018) Branch-and-cut for the split delivery vehicle routing problem with time windows. Transportation Science Forthcoming.

Desaulniers G (2010) Branch-and-price-and-cut for the split delivery vehicle routing problem with time windows. Operations Research 58:179-192, URL http://dx.doi.org/10.1287/opre.1090.0713.

Desaulniers G, Madsen OB, Ropke S (2014) The vehicle routing problem with time windows. Toth P, Vigo D, eds., Vehicle Routing: Problems, Methods, and Applications, 119-159 (Philadelphia: Society for Industrial and Applied Mathematics (SIAM)), URL http://dx.doi.org/10.1137/1.9781611973594. ch5.

Dror M, Trudeau P (1990) Split delivery routing. Naval Research Logistics 37:383-402, URL http://dx. doi.org/10.1002/nav.3800370304.

Fischetti M, Salazar J, Toth P (1995) The symmetric generalized traveling salesman polytope. Networks 26:113-123, URL http://dx.doi.org/10.1002/net.3230260206.

Gendreau M, Dejax P, Feillet D, Gueguen C (2006) Vehicle routing with time windows and split delivery. Technical Report 2006-851, Laboratoire Informatique d'Avignon, Avignon, France.

Groër C, Golden B, Wasil E (2009) The consistent vehicle routing problem. Manufacturing \& Service Operations Management 11(4):630-643, URL http://dx.doi.org/10.1287/msom.1080.0243.

Gulczynski D, Golden B, Wasil E (2010) The split delivery vehicle routing problem with minimum delivery amounts. Transportation Research Part E: Logistics and Transportation Review 46(5):612-626, URL http://dx.doi.org/10.1016/j.tre.2009.12.007.

Irnich S, Schneider M, Vigo D (2014) Four variants of the vehicle routing problem. Toth P, Vigo D, eds., Vehicle Routing: Problems, Methods, and Applications, chapter 9, 241-271 (Philadelphia, PA: Society for Industrial and Applied Mathematics), URL http://dx.doi.org/10.1137/1.9781611973594.ch9.

Kohl N, Desrosiers J, Madsen OBG, Solomon MM, Soumis F (1999) 2-path cuts for the vehicle routing problem with time windows. Transportation Science 33(1):101-116, URL http://dx.doi.org/10. $1287 / \operatorname{trsc} .33 .1 .101$.

Luo Z, Qin H, Zhu W, Lim A (2016) Branch and price and cut for the split-delivery vehicle routing problem with time windows and linear weight-related cost. Transportation Science URL http://dx.doi.org/ $10.1287 / \operatorname{trsc} .2015 .0666$. 
Maffioli F, Sciomachen A (1997) A mixed-integer model for solving ordering problems with side constraints. Annals of Operations Research 69(0):277-297, URL http://dx.doi.org/10.1023/A:1018989130169.

Miller D, Tucker A, Zemlin R (1960) Integer programming formulations of traveling salesman problems. Journal of the ACM 7:326-329, URL http://dx.doi.org/10.1145/321043.321046.

Salazar-González JJ, Santos-Hernández B (2015) The split-demand one-commodity pickup-and-delivery travelling salesman problem. Transportation Research Part B: Methodological 75:58-73, URL http: //dx.doi.org/10.1016/j.trb.2015.02.014.

Solomon MM (1987) Algorithms for the vehicle routing and scheduling problems with time window constraints. Operations Research 35:254-265, URL http://dx.doi.org/10.1287/opre.35.2.254.

van Eijl C (1995) A polyhedral approach to the delivery man problem. Technical Report 95-19, Department of Mathematics and Computer Science, Eindhoven University of Technology.

Wang X, Golden B, Gulczynski D (2014) A worst-case analysis for the split delivery capacitated team orienteering problem with minimum delivery amounts. Optimization Letters 8(8):2349-2356, URL http://dx.doi.org/10.1007/s11590-014-0752-8.

Xiong Y, Gulczynski D, Kleitman D, Golden B, Wasil E (2013) A worst-case analysis for the split delivery vehicle routing problem with minimum delivery amounts. Optimization Letters 7(7):1597-1609, URL http://dx.doi .org/10.1007/s11590-012-0554-9. 


\section{e-Companion}

\section{EC.1. Infeasible-Path and Path-Matching Constraints}

In order to introduce the constraints, some additional notation is required: A path $P=$ $\left(v_{0}, v_{1}, \ldots, v_{\ell}\right)$ is a sequence of vertices with $\left(v_{i-1}, v_{i}\right) \in A$ for $i \in\{1, \ldots, \ell\}$. The start vertex of the path is $s(P)=v_{0}$ and the end vertex is $t(P)=v_{\ell}$. The length of the path is $\ell=\ell(P) \geq 1$. The arcs of $P$ are denoted by $A(P)$, and we define $A_{N}(P)=A(P) \cap A(N)$. The vertices of $P$ are $V(P)=\left\{v_{0}, \ldots, v_{\ell}\right\}$ and the internal vertices are $V^{i n t}(P)=\left\{v_{1}, \ldots, v_{\ell-1}\right\}$. Note that in the SDVRPTW(-IC) the internal vertices of a feasible route are customers, i.e., $V^{\text {int }}(P) \subseteq N$. For the demand of the internal vertices, the shorthand notation $d\left(V^{i n t}(P)\right)$ is used for $\sum_{v \in V^{i n t}(P)} d_{v}$. Paths of length 1 have $V^{\text {int }}(P)=\varnothing$.

A path $P$ with $\left|\left\{v_{1}, \ldots, v_{\ell}\right\}\right|=\left|\left\{v_{0}, v_{1}, \ldots, v_{\ell}-1\right\}\right|=\ell$ is said to be almost-elementary. All the internal vertices of an almost-elementary path are distinct. It is worth noting that, in a solution to the SDVRPTW(-IC), an almost-elementary path represents a subpath of a route in which all the internal vertices are non-split customers. An almost-elementary path $P=\left(v_{0}, v_{1}, \ldots, v_{\ell}\right)$ is timewindow infeasible if there do not exist numbers $T_{0}, T_{1}, \ldots, T_{\ell}$ such that $e_{v_{i}} \leq T_{i} \leq l_{v_{i}}$ holds for all $i=0,1, \ldots, \ell$ and $T_{i-1}+t_{v_{i-1}, v_{i}} \leq T_{i}$ holds for $i=1, \ldots, \ell$. Given an almost-elementary path $P=\left(v_{0}, v_{1}, \ldots, v_{\ell}\right)$, the minimum quantity $\underline{d}(P)$ to deliver along the path is defined as

$$
\underline{d}(P)=\alpha\left(1-\delta_{s(P), 0}\right)+d\left(V^{i n t}(P)\right)+\alpha\left(1-\delta_{t(P), n+1}\right),
$$

where $\delta_{x y} \in\{0,1\}$ is the Kronecker delta which is equal to 1 if $x=y$ and 0 otherwise, and $\alpha \in\{0,1\}$ is equal to 1 if $d_{i} \in \mathbb{Z}_{+}$for all $i \in N$, and $Q \in \mathbb{Z}_{+}$. The $\alpha$-terms exploit Property 5 and define the minimum quantity to deliver. An almost-elementary path $P$ with $P \neq(0, i, n+1)$ for any $i \in N$ is load infeasible if $\underline{d}(P)>Q$. All paths $P=(0, i, n+1)$ with $i \in N$, i.e., of length $\ell(P)=2$, are feasible even if $\underline{d}(P)=d_{i}>Q$.

An almost-elementary path $P=\left(v_{0}, v_{1}, \ldots, v_{\ell}\right)$, is said to be infeasible (for the SDVRPTW(-IC)) if it does not occur as a subpath in any route of a feasible solution to the SDVRPTW(-IC) fulfilling Properties 2-5.

Definition EC.1. An almost-elementary path $P=\left(v_{0}, v_{1}, \ldots, v_{\ell}\right)$, is infeasible if at least one of the following condition is satisfied:

(i) $P$ is time-window infeasible;

(ii) $P$ is load infeasible;

(iii) $P$ is a cycle, i.e. $s(P)=t(P)$. 
Finally, for the presentation of separation procedures, it is assumed that the current (fractional) solution of (3) is given by $\bar{s}=(\overline{\mathbf{x}}, \overline{\mathbf{z}}, \overline{\mathbf{T}})$. Moreover, for any customer $i \in N$ such that $\bar{z}_{i}<1.5, \pi(i)$ and $\sigma(i)$ denote a predecessor and a successor of $i$ in the graph induced by $\bar{s}$, respectively. The different separation procedures use individual tie-breaker rules if predecessors or successors are not unique. For all the customers $i \in N$ such that $\bar{z}_{i} \geq 1.5$, predecessors and successors remain undefined.

\section{EC.1.1. Infeasible-path constraints}

The generalization of infeasible-path constraints first introduced by Ascheuer et al. (2000, 2001) for the TSPTW is as follows:

Proposition EC.1. For all infeasible almost-elementary paths $P$ with $\ell(P) \geq 3$, the infeasiblepath constraint

$$
\sum_{(i, j) \in A_{N}(P)} x_{i j}-\sum_{v \in V^{i n t}(P)} z_{v} \leq-\delta_{s(P), 0}-\delta_{t(P), n+1}
$$

is valid for the polyhedron formed by feasible solutions to the SDVRPTW(-IC) fulfilling Properties 2-5.

Infeasible-path constraints (EC.2) are separated as follows. Recall it is assumed that predecessors $\pi(i)$ and successors $\sigma(i)$ are undefined for customers $i \in N$ such that $\bar{z}_{i} \geq 1.5$. For the other customers, predecessors and successors are initialized using one of the following rules:

$$
\begin{array}{ll}
\pi(i)=\underset{h \in N}{\arg \min }\left\{\left|1-\bar{x}_{h i}\right|\right\}, & \sigma(i)=\underset{j \in N}{\arg \min }\left\{\left|1-\bar{x}_{i j}\right|\right\} ; \\
\pi(i)=\underset{h \in N}{\arg \max }\left\{\max \left(e_{i}, e_{h}+t_{h i}\right) \bar{x}_{h i}\right\}, & \sigma(i)=\underset{j \in N}{\arg \max }\left\{\max \left(e_{j}, e_{i}+t_{i j}\right) \bar{x}_{i j}\right\} ; \\
\pi(i)=\underset{h \in N}{\arg \max }\left\{d_{h} \bar{x}_{h i}\right\}, & \sigma(i)=\underset{j \in N}{\arg \max }\left\{d_{j} \bar{x}_{i j}\right\} .
\end{array}
$$

After the initialization step, for each customer $i \in N$, the almost-elementary path $P=(i)$ is extended iteratively adding predecessors of $s(P)$ or successors of $t(P)$ to the respective endpoint. The extension stops when $s(P)$ has an undefined predecessor and $t(P)$ has an undefined successor. The resulting almost-elementary path $P$, if infeasible (see Definition EC.1), is then checked to violate the corresponding infeasible-path constraint (EC.2) that is eventually added. The separation heuristic is run once for each of the available initialization rules.

\section{EC.1.2. Path-matching constraints}

Path-matching constraints generalize infeasible-path constraints (EC.2).

Definition EC.2. Let $i \in N, p \geq 1$, and $P_{1}^{\text {in }}, P_{2}^{\text {in }}, \ldots, P_{p}^{\text {in }}$ and $P_{1}^{\text {out }}, P_{2}^{\text {out }}, \ldots, P_{p}^{\text {out }}$ be paths with the following properties: 


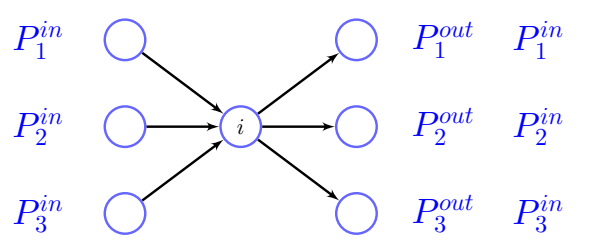

(a)

$P_{1}^{\text {in }}$

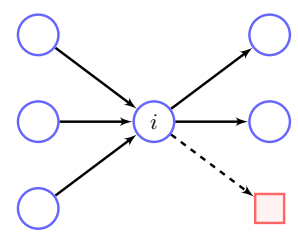

(b)

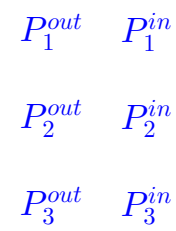

$P_{3}^{\text {out }} P_{3}^{\text {in }}$

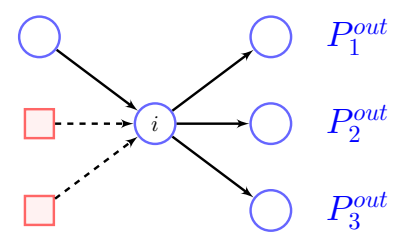

(c)

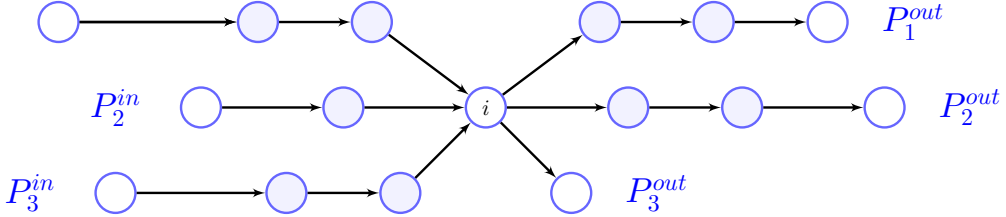

(d)

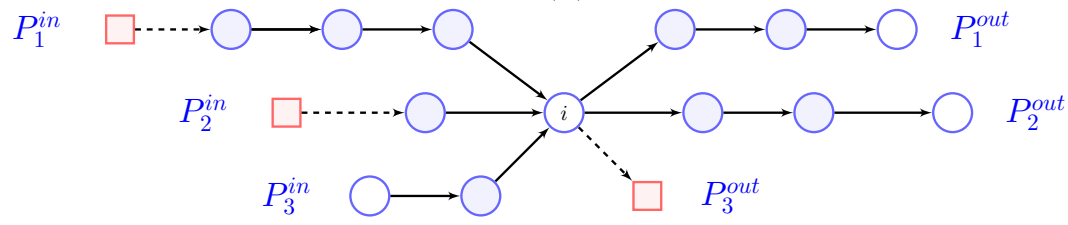

(e)

Figure EC.1 Examples of stretched stars $S\left(i, 3, P^{i n}, P^{o u t}\right)$.

(i) All paths $P_{1}^{i n}, P_{2}^{i n}, \ldots, P_{p}^{i n}$ have end vertex $i$ (in-paths), i.e., $t\left(P_{j}^{i n}\right)=i$ for $1 \leq j \leq p$.

All paths $P_{1}^{\text {out }}, P_{2}^{\text {out }}, \ldots, P_{p}^{\text {out }}$ have start vertex $i$ (out-paths), i.e., $s\left(P_{k}^{\text {out }}\right)=i$ for $1 \leq k \leq p$.

(ii) Internal vertices of all in- and out-paths are disjoint: $V^{i n t}\left(P_{j}^{i n}\right) \cap V^{i n t}\left(P_{j^{\prime}}^{i n}\right)=\varnothing$ for $1 \leq j, j^{\prime} \leq p$, $j \neq j^{\prime} ; V^{\text {int }}\left(P_{k}^{\text {out }}\right) \cap V^{\text {int }}\left(P_{k^{\prime}}^{\text {out }}\right)=\varnothing$ for $1 \leq k, k^{\prime} \leq p, k \neq k^{\prime} ; V^{\text {int }}\left(P_{j}^{\text {in }}\right) \cap V^{\text {int }}\left(P_{k}^{\text {out }}\right)=\varnothing$ for $1 \leq j, k \leq p$. This implies that all concatenations of in-paths and out-paths, in the following denoted by $\left(P_{j}^{\text {in }}, P_{k}^{\text {out }}\right)$, are almost-elementary for all $1 \leq j, k \leq p$.

(iii) If there is an in-path $P_{j}^{\text {in }}=(0, i)$, then $\left(P_{j}^{\text {in }}, P_{k}^{\text {out }}\right)$ is not infeasible for all out-paths $P_{k}^{\text {out }}$ for $1 \leq k \leq p$.

If there is an out-path $P_{k}^{i n}=(i, n+1)$, then $\left(P_{j}^{i n}, P_{k}^{o u t}\right)$ is not infeasible for all in-paths $P_{j}^{i n}$ for $1 \leq j \leq p$.

(iv) In-paths $P_{j}^{i n}=(0, i)$ and out-paths $P_{k}^{i n}=(i, n+1)$ do not occur together.

Such a set of in-paths $P^{\text {in }}=\left\{P_{1}^{\text {in }}, P_{2}^{\text {in }}, \ldots, P_{p}^{\text {in }}\right\}$ and out-paths $P^{\text {out }}=\left\{P_{1}^{\text {out }}, P_{2}^{\text {out }}, \ldots, P_{p}^{\text {out }}\right\}$ is called a stretched star and denoted by $S\left(i, p, P^{\text {in }}, P^{\text {out }}\right)$.

Examples of five different stretched stars are depicted in Figure EC.1.

Given a stretched star $S\left(i, p, P^{\text {in }}, P^{\text {out }}\right)$, any concatenated path $\left(P_{j}^{\text {in }}, P_{k}^{\text {out }}\right)$ for $1 \leq j, k \leq p$ can be tested for infeasibility. While time-window infeasible paths and cycles can be defined as in Definition EC.1, a modified definition of load infeasible paths is required here. It is based on another definition of the minimum quantity $\underline{d}(P)$ to deliver along a path $P$, cf. (EC.1), now defined as

$$
\underline{d}\left(P_{j}^{\text {in }}, P_{k}^{\text {out }}\right)=\alpha\left(1-\delta_{s\left(P_{j}^{i n}\right), 0}\right)+d\left(V^{\text {int }}\left(P_{j}^{\text {in }}\right)\right)+\alpha+d\left(V^{\text {int }}\left(P_{k}^{\text {out }}\right)\right)+\alpha\left(1-\delta_{t\left(P_{k}^{\text {out }}\right), n+1}\right) .
$$


Thus, a path $\left(P_{j}^{\text {in }}, P_{k}^{\text {out }}\right)$ is load infeasible if $\underline{d}\left(P_{j}^{\text {in }}, P_{k}^{\text {out }}\right)>Q$.

Definition EC.3. Let $\left(m_{i j}\right)=M \in\{0,1\}^{m \times n}$ be any binary matrix. Define the associated bipartite graph $B(M)=\left(P \cup W, E_{M}\right)$ with vertices $P=\left\{p_{1}, \ldots, p_{m}\right\}$ and $W=\left\{w_{1}, \ldots, w_{n}\right\}$ (the bipartition), and edges $E_{M}=\left\{\left\{p_{i}, w_{j}\right\}: m_{i j}=1\right.$ for $\left.1 \leq i \leq m, 1 \leq j \leq n\right\}$.

Let $M\left(P^{i n}, P^{o u t}\right)=\left(m_{j k}\right)$ denote the compatibility matrix between the in-paths and out-paths, with $m_{j k}=0$ if path $\left(P_{j}^{i n}, P_{k}^{o u t}\right)$ is infeasible, and $m_{j k}=1$ otherwise. The compatibility number $n_{M}=n_{M}\left(P^{\text {in }}, P^{\text {out }}\right)$ is defined as the size of a maximum-cardinality matching in the bipartite graph $B\left(M\left(P^{\text {in }}, P^{\text {out }}\right)\right)$.

Definition EC.4. A stretched star $S\left(i, p, P^{\text {in }}, P^{\text {out }}\right)$ is called infeasible if $n_{M}\left(P^{\text {in }}, P^{\text {out }}\right)<p$.

Define the number $n_{D}=n_{D}\left(P^{\text {in }}, P^{\text {out }}\right)$ of paths with a depot in the stretched star $S\left(i, p, P^{\text {in }}, P^{\text {out }}\right)$ by $n_{D}=\left|\left\{j: 1 \leq j \leq p, s\left(P_{j}^{\text {in }}\right)=0\right\}\right|+\left|\left\{k: 1 \leq k \leq p, t\left(P_{k}^{\text {out }}\right)=n+1\right\}\right|$.

THEOREM EC.1. For all infeasible stretched stars $S\left(i, p, P^{i n}, P^{\text {out }}\right)$, the path-matching constraint

$$
\sum_{j=1}^{p}\left(\sum_{(g, h) \in A_{N}\left(P_{j}^{\text {in }}\right)} x_{g h}-\sum_{v \in V^{\text {int }}\left(P_{j}^{\text {in }}\right)} z_{v}\right)+\sum_{k=1}^{p}\left(\sum_{(g, h) \in A_{N}\left(P_{k}^{\text {out }}\right)} x_{g h}-\sum_{v \in V^{\text {int }}\left(P_{k}^{\text {out }}\right)} z_{v}\right)-z_{i} \leq n_{M}-n_{D}
$$

with $n_{M}=n_{M}\left(P^{\text {in }}, P^{\text {out }}\right)$ and $n_{D}=n_{D}\left(P^{\text {in }}, P^{\text {out }}\right)$ is valid for the polyhedron formed by feasible solutions to the SDVRPTW(-IC) fulfilling Properties $2-5$.

Path-matching constraints (EC.4) are separated as follows. For each customer $i \in N, p=$ $p(i)=\left\lfloor z_{i}+\frac{1}{2}\right\rfloor$ is computed, and, if $p \geq 2$, violated inequalities for stretched stars of the form $S\left(i, p, P^{i n}, P^{o u t}\right)$ are searched for. Tentative in-paths $P_{1}^{i n}, \ldots, P_{p}^{\text {in }}$ and out-paths $P_{1}^{\text {out }}, \ldots, P_{p}^{\text {out }}$ are iteratively constructed. Initially, all in-paths and out-path consist of single arcs only (like in Figure EC.1 $(\mathrm{a}-\mathrm{c}))$ resulting from the $p \operatorname{arcs}(v, i) \in \Gamma^{-}(i)$ and the $p \operatorname{arcs}(i, v) \in \Gamma^{+}(i)$ with maximum flow $\bar{x}_{v i}$ and $\bar{x}_{i v}$ (depot arcs with flow greater than 1 can lead to multiple copies of these arcs). In each iteration, it is first tested whether the current stretched $\operatorname{star} S\left(i, p, P^{\text {in }}, P^{\text {out }}\right)$ imposes a violated path-matching constraint. To do this, the difference between left-hand and right-hand side of (EC.4) is computed. This requires to determine $n_{M}$ for $M=M\left(P^{\text {in }}, P^{\text {out }}\right)$, i.e., to compute the compatibility matrix $M$ and then solve a (small) matching/assignment problem using a network flow solver. If $n_{M}=p$ the next steps for computing the possible violation of (EC.4) can be skipped. Otherwise (the stretched star is infeasible in this case), the values of the left-hand side of (EC.4) and of $n_{D}=n_{D}\left(P^{i n}, P^{o u t}\right)$ are computed, and separation terminates if the inequality is violated. This latter computation is rather simple because from one iteration to the next, only a single arc is always added to only one of the in-paths or out-paths. This next arc is one giving the highest contribution to the left-hand side of the path-matching constraint (EC.4). More precisely, for $\operatorname{arcs}(g, h) \in A$ that can extend an in-path $P_{j}^{i n}$, i.e., $h=s\left(P_{j}^{i n}\right)$ for some $j \in\{1,2, \ldots, p\}$, the 
contribution is $\bar{x}_{g h}-\bar{z}_{h}$, while $\operatorname{arcs}(g, h) \in A$ that can extend an out-path $P_{k}^{\text {out }}$, i.e., $g=t\left(P_{k}^{\text {out }}\right)$ for some $k \in\{1,2, \ldots, p\}$, the contribution is $\bar{x}_{g h}-\bar{z}_{g}$. Moreover, it is required that $g=\pi(h)$ for in-paths and $\sigma(g)=h$ for out-paths to make the extensions unique, where predecessors and successors are defined as in Section EC.1.1 by Rule 1; this also includes that all internal vertices $v \in V^{\text {int }}\left(P_{j}^{\text {in }}\right) \cup V^{\text {int }}\left(P_{k}^{\text {out }}\right)$ fulfill $\bar{z}_{v}<1.5$. Iterations stop as soon as a violated constraint is found or all in-paths have no predecessor $\pi\left(s\left(P_{j}^{i n}\right)\right)$ of their start vertex $s\left(P_{j}^{i n}\right)$ and all out-paths have no successor $\sigma\left(t\left(P_{k}^{o u t}\right)\right)$ of their last vertex $t\left(P_{k}^{\text {out }}\right)$.

\section{EC.2. Results grouped by Demand Scenario}

The subsequent Tables EC.1-EC.9 provide further details on the aggregated results given in Tables 4, 5, and 6. Tables EC.1-EC.3 show the effects of the different objective functions on the solution structure of the different SDVRPTW policies compared to the VRPTW, and Tables EC.4EC.6 indicate the relative savings obtained with the different objective functions. Tables EC.1-EC.6 are based on the 115 instances solved to optimality with the three objective functions. These are divided between the different demand scenarios as follows:
D1 42
D2 51
D3 14
D4 8
D5 0

This means that scenario D5 (the one having the highest average customer demand in relation to vehicle capacity) is clearly the hardest to solve, and scenarios D3 and D4 are still considerably more difficult than D1 and D2. For obvious reasons, scenario D5 is omitted from Tables EC.1-EC.6.

Tables EC.7-EC.9 describe the impact of inconvenience constraints on the performance of the branch-and-cut algorithm. The results given in these tables were obtained using, for each objective, the instances that were solved to optimality with the SDVRPTW policy for this objective. This is because, as explained in Section 5.2, the TNVx policies can, by definition, be applied to these instances only.

The following observations can be made in Tables EC.1-EC.9:

- The number of visits and the percentage of split customers is highest for scenarios D3 and D4. This is to be expected, as these scenarios have a higher ratio of customer demand to vehicle capacity.

- For objective I, the number of customers with deliveries fully synchronized is by far highest for scenario D4. For objectives II and III, this value is, in general, highest for scenario D3 (note, however, that there are no split customers at all for scenario D4 instances with objective II).

- For objectives I and III, the timespan between the first and last delivery in relation to the time window width is highest for scenario D3. For objective II, there is no discernible pattern. 
- Savings are generally highest for scenario D3.

- D5 instances are among the hardest to solve for all objectives, especially with the TNVx policies. In particular, for objectives I and III, only 1, 2, and 29 instances (out of 78), and 1, 0, and 1 instances (out of 32) were respectively solved to optimality with the TNVx policies. It is also noteworthy that, for scenario D5, no violated 2-path cuts were found, and only 3 violated connectivity cuts.

Summing up, the results suggest that scenario D3, i.e., a demand pattern where the average demand of a customer is between 30 and $70 \%$ of the vehicle capacity, is particularly promising for split delivery distribution strategies. 
Table EC.1 Objective I - Effect of the different distribution policies on solution structure compared to VRPTW, grouped by demand scenario

\begin{tabular}{|c|c|c|c|c|c|}
\hline \multirow[b]{2}{*}{$\begin{array}{l}\text { Policy/ } \\
\text { Demand } \\
\text { scenario }\end{array}$} & \multicolumn{5}{|c|}{ Average of } \\
\hline & $\begin{array}{l}\text { Number of } \\
\text { visits per } \\
\text { customer }\end{array}$ & $\begin{array}{c}\text { Percentage } \\
\text { of split } \\
\text { customers }\end{array}$ & $\begin{array}{l}\text { Number of } \\
\text { visits per } \\
\text { split } \\
\text { customer }\end{array}$ & $\begin{array}{c}\text { Percentage of } \\
\text { split customers } \\
\text { with deliveries } \\
\text { fully } \\
\text { synchronized }\end{array}$ & $\begin{array}{c}\text { Timespan } \\
\text { between first and } \\
\text { last delivery in } \\
\text { relation to time } \\
\text { window width in } \\
\%\end{array}$ \\
\hline \multicolumn{6}{|l|}{ SDVRPTW } \\
\hline D1 & 1.10 & 9.95 & 2.00 & 22.22 & 25.26 \\
\hline D2 & 1.06 & 6.00 & 2.00 & 7.71 & 30.77 \\
\hline D3 & 1.21 & 21.43 & 2.00 & 29.44 & 41.78 \\
\hline D4 & 1.14 & 13.50 & 2.00 & 65.63 & 21.25 \\
\hline Avg. & 1.10 & 9.84 & 2.00 & 20.95 & 29.44 \\
\hline \multicolumn{6}{|l|}{ NV2 } \\
\hline D1 & 1.10 & 9.95 & 2.00 & 22.62 & 23.76 \\
\hline D2 & 1.06 & 6.31 & 2.00 & 8.75 & 29.56 \\
\hline D3 & 1.21 & 21.43 & 2.00 & 28.42 & 41.66 \\
\hline D4 & 1.14 & 13.50 & 2.00 & 65.63 & 21.73 \\
\hline Avg. & 1.10 & 9.98 & 2.00 & 21.37 & 28.37 \\
\hline \multicolumn{6}{|l|}{ So } \\
\hline D1 & 1.10 & 9.95 & 2.00 & 100.00 & 0.00 \\
\hline D2 & 1.06 & 6.04 & 2.00 & 100.00 & 0.00 \\
\hline D3 & 1.21 & 21.43 & 2.00 & 100.00 & 0.00 \\
\hline D4 & 1.14 & 13.50 & 2.00 & 100.00 & 0.00 \\
\hline Avg. & 1.10 & 9.86 & 2.00 & 100.00 & 0.00 \\
\hline \multicolumn{6}{|l|}{ TNV25 } \\
\hline D1 & 1.04 & 3.67 & 2.00 & 10.26 & 31.38 \\
\hline D2 & 1.02 & 1.76 & 2.00 & 28.00 & 10.66 \\
\hline D3 & 1.05 & 5.43 & 2.00 & 14.29 & 57.93 \\
\hline D4 & 1.04 & 4.00 & 2.00 & 75.00 & 12.57 \\
\hline Avg. & 1.03 & 3.06 & 2.00 & 22.09 & 24.11 \\
\hline \multicolumn{6}{|l|}{ TNV50 } \\
\hline D1 & 1.05 & 5.19 & 2.00 & 16.67 & 34.44 \\
\hline D2 & 1.04 & 3.88 & 2.00 & 16.25 & 26.03 \\
\hline D3 & 1.12 & 11.14 & 2.14 & 30.36 & 42.98 \\
\hline $\mathrm{D} 4$ & 1.08 & 8.00 & 2.00 & 62.50 & 17.87 \\
\hline Avg. & 1.06 & 5.53 & 2.02 & 21.88 & 30.60 \\
\hline \multicolumn{6}{|l|}{ TNV75 } \\
\hline D1 & 1.09 & 8.76 & 2.00 & 22.02 & 25.88 \\
\hline D2 & 1.05 & 4.59 & 2.00 & 12.08 & 27.20 \\
\hline D3 & 1.16 & 16.00 & 2.00 & 17.50 & 46.12 \\
\hline D4 & 1.10 & 9.50 & 2.00 & 70.83 & 14.95 \\
\hline Avg. & 1.08 & 7.84 & 2.00 & 21.35 & 28.17 \\
\hline
\end{tabular}


Table EC.2 Objective II - Effect of the different distribution policies on solution structure compared to VRPTW, grouped by demand scenario

\begin{tabular}{|c|c|c|c|c|c|}
\hline \multirow[b]{2}{*}{$\begin{array}{l}\text { Policy/ } \\
\text { Demand } \\
\text { scenario }\end{array}$} & \multicolumn{5}{|c|}{ Average of } \\
\hline & $\begin{array}{l}\text { Number of } \\
\text { visits per } \\
\text { customer }\end{array}$ & $\begin{array}{c}\text { Percentage } \\
\text { of split } \\
\text { customers }\end{array}$ & $\begin{array}{l}\text { Number of } \\
\text { visits per } \\
\text { split } \\
\text { customer }\end{array}$ & $\begin{array}{c}\text { Percentage of } \\
\text { split customers } \\
\text { with deliveries } \\
\text { fully } \\
\text { synchronized }\end{array}$ & $\begin{array}{c}\text { Timespan } \\
\text { between first and } \\
\text { last delivery in } \\
\text { relation to time } \\
\text { window width in } \\
\%\end{array}$ \\
\hline \multicolumn{6}{|l|}{ SDVRPTW } \\
\hline D1 & 1.04 & 3.90 & 2.00 & 13.16 & 17.21 \\
\hline D2 & 1.03 & 2.51 & 2.00 & 5.00 & 12.26 \\
\hline D3 & 1.04 & 3.71 & 2.08 & 16.67 & 16.73 \\
\hline D4 & 1.00 & 0.00 & n.a. & n.a. & n.a. \\
\hline Avg. & 1.03 & 2.99 & 2.01 & 10.00 & 13.76 \\
\hline $\mathbf{N V 2}$ & 1.04 & 3.90 & 2.00 & 13.16 & 16.25 \\
\hline D2 & 1.03 & 2.51 & 2.00 & 5.00 & 13.88 \\
\hline D3 & 1.04 & 3.71 & 2.00 & 22.22 & 17.44 \\
\hline $\mathrm{D} 4$ & 1.00 & 0.00 & n.a. & $n . a$. & $n . a$. \\
\hline Avg. & 1.03 & 2.99 & 2.00 & 10.74 & 14.21 \\
\hline \multicolumn{6}{|l|}{ So } \\
\hline D1 & 1.03 & 2.95 & 2.00 & 100.00 & 0.00 \\
\hline D2 & 1.02 & 2.04 & 2.00 & 100.00 & 0.00 \\
\hline D3 & 1.04 & 3.43 & 2.08 & 100.00 & 0.00 \\
\hline D4 & 1.00 & 0.00 & n.a. & n.a. & n.a. \\
\hline Avg. & 1.02 & 2.40 & 2.01 & 100.00 & 0.00 \\
\hline \multicolumn{6}{|l|}{ TNV25 } \\
\hline D1 & 1.01 & 1.10 & 2.00 & 16.67 & 10.07 \\
\hline D2 & 1.01 & 0.94 & 2.00 & 8.33 & 10.40 \\
\hline D3 & 1.01 & 0.57 & 2.00 & 50.00 & 5.41 \\
\hline D4 & 1.00 & 0.00 & n.a. & $n . a$. & n.a. \\
\hline Avg. & 1.01 & 0.89 & 2.00 & 15.38 & 8.95 \\
\hline \multicolumn{6}{|l|}{ TNV50 } \\
\hline D1 & 1.02 & 2.24 & 2.00 & 18.42 & 13.90 \\
\hline D2 & 1.02 & 1.57 & 2.00 & 5.00 & 15.81 \\
\hline D3 & 1.01 & 1.14 & 2.00 & 0.00 & 6.39 \\
\hline D4 & 1.00 & 0.00 & n.a. & n.a. & n.a. \\
\hline Avg. & 1.02 & 1.65 & 2.00 & 10.98 & 12.86 \\
\hline \multicolumn{6}{|l|}{ TNV75 } \\
\hline D1 & 1.02 & 2.29 & 2.00 & 13.16 & 15.45 \\
\hline D2 & 1.03 & 2.51 & 2.00 & 5.00 & 12.18 \\
\hline D3 & 1.03 & 3.43 & 2.00 & 16.67 & 14.87 \\
\hline D4 & 1.00 & 0.00 & n.a. & n.a. & n.a. \\
\hline Avg. & 1.02 & 2.37 & 2.00 & 10.00 & 12.85 \\
\hline
\end{tabular}


Table EC.3 Objective III - Effect of the different distribution policies on solution structure compared to VRPTW, grouped by demand scenario

\begin{tabular}{|c|c|c|c|c|c|}
\hline \multirow[b]{2}{*}{$\begin{array}{l}\text { Policy/ } \\
\text { Demand } \\
\text { scenario }\end{array}$} & \multicolumn{5}{|c|}{ Average of } \\
\hline & $\begin{array}{l}\text { Number of } \\
\text { visits per } \\
\text { customer }\end{array}$ & $\begin{array}{c}\text { Percentage } \\
\text { of split } \\
\text { customers }\end{array}$ & $\begin{array}{l}\text { Number of } \\
\text { visits per } \\
\text { split } \\
\text { customer }\end{array}$ & $\begin{array}{c}\text { Percentage of } \\
\text { split customers } \\
\text { with deliveries } \\
\text { fully } \\
\text { synchronized }\end{array}$ & $\begin{array}{c}\text { Timespan } \\
\text { between first and } \\
\text { last delivery in } \\
\text { relation to time } \\
\text { window width in } \\
\%\end{array}$ \\
\hline \multicolumn{6}{|l|}{ SDVRPTW } \\
\hline D1 & 1.05 & 4.57 & 2.00 & 18.12 & 19.69 \\
\hline D2 & 1.03 & 2.67 & 2.00 & 9.09 & 12.24 \\
\hline D3 & 1.07 & 6.29 & 2.04 & 37.18 & 30.86 \\
\hline $\mathrm{D} 4$ & 1.02 & 1.50 & 2.00 & 25.00 & 11.63 \\
\hline Avg. & 1.04 & 3.72 & 2.01 & 19.17 & 17.18 \\
\hline \multicolumn{6}{|l|}{ NV2 } \\
\hline D1 & 1.05 & 4.57 & 2.00 & 15.22 & 20.73 \\
\hline D2 & 1.03 & 2.67 & 2.00 & 6.82 & 11.82 \\
\hline D3 & 1.06 & 6.29 & 2.00 & 14.10 & 42.66 \\
\hline $\mathrm{D} 4$ & 1.02 & 1.50 & 2.00 & 0.00 & 17.88 \\
\hline Avg. & 1.04 & 3.72 & 2.00 & 11.39 & 19.25 \\
\hline \multicolumn{6}{|l|}{ So } \\
\hline D1 & 1.04 & 3.52 & 2.00 & 100.00 & 0.00 \\
\hline D2 & 1.02 & 2.20 & 2.00 & 100.00 & 0.00 \\
\hline D3 & 1.06 & 6.00 & 2.04 & 100.00 & 0.00 \\
\hline $\mathrm{D} 4$ & 1.02 & 1.50 & 2.00 & 100.00 & 0.00 \\
\hline Avg. & 1.03 & 3.10 & 2.01 & 100.00 & 0.00 \\
\hline \multicolumn{6}{|l|}{ TNV25 } \\
\hline D1 & 1.01 & 1.19 & 2.00 & 15.38 & 6.95 \\
\hline D2 & 1.01 & 0.94 & 2.00 & 8.33 & 8.29 \\
\hline D3 & 1.02 & 2.00 & 2.00 & 28.57 & 15.11 \\
\hline $\mathrm{D} 4$ & 1.01 & 0.50 & 2.00 & 0.00 & 12.49 \\
\hline Avg. & 1.01 & 1.13 & 2.00 & 15.15 & 8.92 \\
\hline \multicolumn{6}{|l|}{ TNV50 } \\
\hline D1 & 1.03 & 3.19 & 2.00 & 17.39 & 18.71 \\
\hline D2 & 1.02 & 1.73 & 2.00 & 9.09 & 14.65 \\
\hline D3 & 1.05 & 4.57 & 2.00 & 38.46 & 25.73 \\
\hline $\mathrm{D} 4$ & 1.01 & 1.00 & 2.00 & 0.00 & 17.88 \\
\hline$A v g$. & 1.03 & 2.56 & 2.00 & 18.33 & 17.71 \\
\hline \multicolumn{6}{|l|}{ TNV75 } \\
\hline D1 & 1.03 & 3.33 & 2.00 & 10.87 & 21.46 \\
\hline D2 & 1.03 & 2.67 & 2.00 & 6.82 & 12.19 \\
\hline D3 & 1.06 & 5.71 & 2.00 & 38.46 & 23.28 \\
\hline D4 & 1.02 & 1.50 & 2.00 & 0.00 & 17.88 \\
\hline Avg. & 1.03 & 3.20 & 2.00 & 15.00 & 17.32 \\
\hline
\end{tabular}


Table EC.4 Objective I - Relative savings obtained with the different distribution policies compared to VRPTW, grouped by demand scenario

\begin{tabular}{|c|c|c|c|c|c|}
\hline \multirow[b]{2}{*}{$\begin{array}{l}\text { Policy / } \\
\text { Demand } \\
\text { scenario }\end{array}$} & \multicolumn{5}{|c|}{ Min./Avg./Max. \% Savings/\# Instances with savings $>3 \%$ in } \\
\hline & Objective value & Number of routes & $\begin{array}{l}\text { Variable routing } \\
\text { costs }\end{array}$ & Route durations & $\begin{array}{l}\text { Sum of variable } \\
\text { routing costs and } \\
\text { costs related to } \\
\text { route durations }\end{array}$ \\
\hline \multicolumn{6}{|l|}{ SDVRPTW } \\
\hline D1 & $0.44 / 2.37 / 4.61 / 19$ & $0.00 / 0.63 / 9.09 / 3$ & $0.44 / 2.37 / 4.61 / 19$ & $-28.33 /-4.70 / 16.99 / 6$ & $-24.58 /-2.91 / 15.84 / 6$ \\
\hline D2 & $0.00 / 1.29 / 3.29 / 6$ & $0.00 / 0.65 / 6.67 / 5$ & $0.00 / 1.29 / 3.29 / 6$ & $-81.47 /-4.28 / 8.99 / 2$ & $-70.56 /-3.19 / 7.12 / 2$ \\
\hline D3 & $3.55 / 6.45 / 8.87 / 14$ & $7.14 / 8.47 / 13.33 / 14$ & $3.55 / 6.45 / 8.87 / 14$ & $-47.37 /-13.07 / 0.73 / 0$ & $-28.79 /-9.12 / 1.99 / 0$ \\
\hline D4 & $3.39 / 4.70 / 5.23 / 8$ & $10.00 / 10.00 / 10.00 / 8$ & $3.39 / 4.70 / 5.23 / 8$ & $-58.50 /-38.91 /-5.21 / 0$ & $-49.71 /-32.81 /-4.62 / 0$ \\
\hline Avg. & $0.00 / 2.55 / 8.87 / 47$ & $0.00 / 2.25 / 13.33 / 30$ & $0.00 / 2.55 / 8.87 / 47$ & $-81.47 /-7.91 / 16.99 / 8$ & $-70.56 /-5.87 / 15.84 / 8$ \\
\hline \multicolumn{6}{|l|}{ NV2 } \\
\hline D1 & $0.44 / 2.37 / 4.61 / 19$ & $0.00 / 0.63 / 9.09 / 3$ & $0.44 / 2.37 / 4.61 / 19$ & $-28.33 /-4.72 / 18.46 / 6$ & $-24.58 /-2.91 / 17.19 / 6$ \\
\hline D2 & $0.00 / 1.29 / 3.29 / 6$ & $0.00 / 0.65 / 6.67 / 5$ & $0.00 / 1.29 / 3.29 / 6$ & $-81.47 /-4.41 / 8.99 / 3$ & $-70.56 /-3.32 / 7.12 / 3$ \\
\hline D3 & $3.55 / 6.45 / 8.87 / 14$ & $7.14 / 8.47 / 13.33 / 14$ & $3.55 / 6.45 / 8.87 / 14$ & $-47.37 /-13.07 / 0.73 / 0$ & $-28.79 /-9.12 / 1.99 / 0$ \\
\hline D4 & $3.39 / 4.70 / 5.23 / 8$ & $10.00 / 10.00 / 10.00 / 8$ & $3.39 / 4.70 / 5.23 / 8$ & $-58.50 /-38.05 / 1.70 / 0$ & $-49.71 /-32.00 / 1.82 / 0$ \\
\hline$A v g$ & $0.00 / 2.55 / 8.87 / 47$ & $0.00 / 2.25 / 13.33 / 30$ & $0.00 / 2.55 / 8.87 / 47$ & $-81.47 /-7.92 / 18.46 / 9$ & $-70.56 /-5.87 / 17.19 / 9$ \\
\hline \multicolumn{6}{|l|}{ So } \\
\hline D1 & $0.44 / 2.29 / 4.00 / 17$ & $0.00 / 0.63 / 9.09 / 3$ & $0.44 / 2.29 / 4.00 / 17$ & $-273.23 /-54.18 / 2.31 / 0$ & $-233.31 /-37.72 / 2.39 / 0$ \\
\hline D2 & $0.00 / 1.25 / 3.13 / 3$ & $0.00 / 0.65 / 6.67 / 5$ & $0.00 / 1.25 / 3.13 / 3$ & $-350.49 /-50.33 / 0.00 / 0$ & $-300.42 /-37.84 / 0.00 / 0$ \\
\hline D3 & $3.55 / 6.45 / 8.87 / 14$ & $7.14 / 8.47 / 13.33 / 14$ & $3.55 / 6.45 / 8.87 / 14$ & $-119.93 /-44.79 /-16.20 / 0$ & $-101.25 /-33.40 /-7.64 / 0$ \\
\hline D4 & $3.39 / 4.70 / 5.23 / 8$ & $10.00 / 10.00 / 10.00 / 8$ & $3.39 / 4.70 / 5.23 / 8$ & $-402.52 /-97.72 /-8.19 / 0$ & $-332.27 /-81.92 /-7.40 / 0$ \\
\hline Avg. & $0.00 / 2.50 / 8.87 / 42$ & $0.00 / 2.25 / 13.33 / 30$ & $0.00 / 2.50 / 8.87 / 42$ & $-402.52 /-54.36 / 2.31 / 0$ & $-332.27 /-40.32 / 2.39 / 0$ \\
\hline \multicolumn{6}{|l|}{ TNV25 } \\
\hline D1 & $0.00 / 1.28 / 3.50 / 5$ & $-10.00 /-2.38 / 0.00 / 0$ & $0.00 / 1.28 / 3.50 / 5$ & $-17.66 /-1.26 / 24.48 / 5$ & $-15.28 /-0.83 / 18.49 / 5$ \\
\hline D2 & $0.00 / 0.65 / 2.00 / 0$ & $0.00 / 0.00 / 0.00 / 0$ & $0.00 / 0.65 / 2.00 / 0$ & $-34.49 /-1.90 / 7.39 / 3$ & $-27.45 /-1.47 / 5.94 / 1$ \\
\hline D3 & $0.23 / 2.68 / 5.49 / 5$ & $0.00 / 1.43 / 6.67 / 3$ & $0.23 / 2.68 / 5.49 / 5$ & $-16.42 / 3.16 / 27.69 / 5$ & $-13.85 / 3.59 / 25.14 / 5$ \\
\hline D4 & $1.63 / 3.12 / 3.57 / 6$ & $10.00 / 10.00 / 10.00 / 8$ & $1.63 / 3.12 / 3.57 / 6$ & $-46.82 /-35.97 /-22.61 / 0$ & $-38.40 /-30.61 /-19.15 / 0$ \\
\hline Avg. & $0.00 / 1.30 / 5.49 / 16$ & $-10.00 / 0.00 / 10.00 / 11$ & $0.00 / 1.30 / 5.49 / 16$ & $-46.82 /-3.42 / 27.69 / 13$ & $-38.40 /-2.65 / 25.14 / 11$ \\
\hline \multicolumn{6}{|l|}{ TNV50 } \\
\hline D1 & $0.37 / 1.82 / 3.73 / 7$ & $0.00 / 0.41 / 9.09 / 2$ & $0.37 / 1.82 / 3.73 / 7$ & $-17.66 /-1.89 / 18.46 / 5$ & $-15.28 /-0.87 / 17.19 / 5$ \\
\hline D2 & $0.00 / 1.10 / 2.70 / 0$ & $0.00 / 0.52 / 6.67 / 4$ & $0.00 / 1.10 / 2.70 / 0$ & $-34.49 /-1.67 / 34.26 / 7$ & $-27.45 /-1.39 / 24.15 / 5$ \\
\hline D3 & $2.99 / 5.83 / 8.19 / 11$ & $7.14 / 8.47 / 13.33 / 14$ & $2.99 / 5.83 / 8.19 / 11$ & $-28.65 /-11.65 / 0.95 / 0$ & $-18.42 /-8.50 / 1.86 / 0$ \\
\hline D4 & $2.70 / 4.15 / 4.59 / 7$ & $10.00 / 10.00 / 10.00 / 8$ & $2.70 / 4.15 / 4.59 / 7$ & $-42.10 /-28.17 /-6.89 / 0$ & $-35.64 /-23.66 /-6.23 / 0$ \\
\hline Avg. & $0.00 / 2.15 / 8.19 / 25$ & $0.00 / 2.11 / 13.33 / 28$ & $0.00 / 2.15 / 8.19 / 25$ & $-42.10 /-4.81 / 34.26 / 12$ & $-35.64 /-3.62 / 24.15 / 10$ \\
\hline \multicolumn{6}{|l|}{ TNV75 } \\
\hline D1 & $0.44 / 2.31 / 4.41 / 19$ & $0.00 / 0.63 / 9.09 / 3$ & $0.44 / 2.31 / 4.41 / 19$ & $-28.33 /-3.94 / 18.46 / 6$ & $-24.58 /-2.47 / 17.19 / 6$ \\
\hline D2 & $0.00 / 1.19 / 2.77 / 0$ & $0.00 / 0.52 / 6.67 / 4$ & $0.00 / 1.19 / 2.77 / 0$ & $-31.76 /-2.29 / 34.26 / 6$ & $-27.45 /-1.74 / 24.15 / 5$ \\
\hline D3 & $3.40 / 6.16 / 8.45 / 14$ & $7.14 / 8.47 / 13.33 / 14$ & $3.40 / 6.16 / 8.45 / 14$ & $-47.55 /-14.83 / 0.55 / 0$ & $-28.96 /-10.70 / 1.82 / 0$ \\
\hline $\mathrm{D} 4$ & $2.70 / 4.34 / 4.97 / 7$ & $10.00 / 10.00 / 10.00 / 8$ & $2.70 / 4.34 / 4.97 / 7$ & $-56.30 /-31.57 /-6.89 / 0$ & $-47.81 /-26.55 /-6.23 / 0$ \\
\hline Avg. & $0.00 / 2.42 / 8.45 / 40$ & $0.00 / 2.19 / 13.33 / 29$ & $0.00 / 2.42 / 8.45 / 40$ & $-56.30 /-6.46 / 34.26 / 12$ & $-47.81 /-4.82 / 24.15 / 11$ \\
\hline
\end{tabular}


Table EC.5 Objective II - Relative savings obtained with the different distribution policies compared to VRPTW, grouped by demand scenario

\begin{tabular}{|c|c|c|c|c|c|}
\hline \multirow[b]{2}{*}{$\begin{array}{l}\text { Policy/ } \\
\text { Demand } \\
\text { scenario }\end{array}$} & \multicolumn{5}{|c|}{ Min./Avg./Max. \% Savings/\# Instances with savings $>3 \%$ in } \\
\hline & Objective value & Number of routes & $\begin{array}{c}\text { Variable routing } \\
\text { costs }\end{array}$ & Route durations & $\begin{array}{l}\text { Sum of variable } \\
\text { routing costs and } \\
\text { costs related to } \\
\text { route durations }\end{array}$ \\
\hline \multicolumn{6}{|l|}{ SDVRPTW } \\
\hline D1 & $0.00 / 0.51 / 1.93 / 0$ & $0.00 / 1.28 / 9.09 / 6$ & $-1.15 / 1.16 / 4.34 / 11$ & $-1.38 / 0.03 / 2.01 / 0$ & $0.00 / 0.51 / 1.93 / 0$ \\
\hline D2 & $0.00 / 0.42 / 1.94 / 0$ & $0.00 / 0.75 / 18.18 / 3$ & $0.00 / 1.01 / 4.86 / 7$ & $-2.34 /-0.01 / 0.95 / 0$ & $0.00 / 0.42 / 1.94 / 0$ \\
\hline D3 & $0.00 / 0.80 / 2.07 / 0$ & $0.00 / 3.06 / 7.14 / 6$ & $0.00 / 1.30 / 3.60 / 3$ & $0.00 / 0.38 / 1.16 / 0$ & $0.00 / 0.80 / 2.07 / 0$ \\
\hline D4 & $0.00 / 0.00 / 0.00 / 0$ & $0.00 / 0.00 / 0.00 / 0$ & $0.00 / 0.00 / 0.00 / 0$ & $0.00 / 0.00 / 0.00 / 0$ & $0.00 / 0.00 / 0.00 / 0$ \\
\hline Avg. & $0.00 / 0.47 / 2.07 / 0$ & $0.00 / 1.17 / 18.18 / 15$ & $-1.15 / 1.03 / 4.86 / 21$ & $-2.34 / 0.06 / 2.01 / 0$ & $0.00 / 0.47 / 2.07 / 0$ \\
\hline \multicolumn{6}{|l|}{ NV2 } \\
\hline D1 & $0.00 / 0.51 / 1.93 / 0$ & $0.00 / 1.28 / 9.09 / 6$ & $-1.15 / 1.16 / 4.34 / 11$ & $-1.38 / 0.03 / 2.01 / 0$ & $0.00 / 0.51 / 1.93 / 0$ \\
\hline D2 & $0.00 / 0.42 / 1.94 / 0$ & $0.00 / 0.75 / 18.18 / 3$ & $0.00 / 1.01 / 4.86 / 7$ & $-2.34 /-0.01 / 0.95 / 0$ & $0.00 / 0.42 / 1.94 / 0$ \\
\hline D3 & $0.00 / 0.80 / 2.07 / 0$ & $0.00 / 3.06 / 7.14 / 6$ & $0.00 / 1.27 / 3.18 / 3$ & $0.00 / 0.41 / 1.16 / 0$ & $0.00 / 0.80 / 2.07 / 0$ \\
\hline D4 & $0.00 / 0.00 / 0.00 / 0$ & $0.00 / 0.00 / 0.00 / 0$ & $0.00 / 0.00 / 0.00 / 0$ & $0.00 / 0.00 / 0.00 / 0$ & $0.00 / 0.00 / 0.00 / 0$ \\
\hline Avg. & $0.00 / 0.47 / 2.07 / 0$ & $0.00 / 1.17 / 18.18 / 15$ & $-1.15 / 1.02 / 4.86 / 21$ & $-2.34 / 0.06 / 2.01 / 0$ & $0.00 / 0.47 / 2.07 / 0$ \\
\hline \multicolumn{6}{|l|}{ So } \\
\hline D1 & $0.00 / 0.43 / 1.93 / 0$ & $-10.00 / 0.80 / 9.09 / 6$ & $0.00 / 1.00 / 4.34 / 8$ & $-1.19 / 0.00 / 1.72 / 0$ & $0.00 / 0.43 / 1.93 / 0$ \\
\hline $\mathrm{D} 2$ & $0.00 / 0.34 / 1.85 / 0$ & $0.00 / 0.75 / 18.18 / 3$ & $0.00 / 0.87 / 4.86 / 6$ & $-2.85 /-0.04 / 0.95 / 0$ & $0.00 / 0.34 / 1.85 / 0$ \\
\hline D3 & $0.00 / 0.77 / 2.06 / 0$ & $0.00 / 3.06 / 7.14 / 6$ & $0.00 / 1.27 / 3.18 / 3$ & $0.00 / 0.36 / 1.16 / 0$ & $0.00 / 0.77 / 2.06 / 0$ \\
\hline D4 & $0.00 / 0.00 / 0.00 / 0$ & $0.00 / 0.00 / 0.00 / 0$ & $0.00 / 0.00 / 0.00 / 0$ & $0.00 / 0.00 / 0.00 / 0$ & $0.00 / 0.00 / 0.00 / 0$ \\
\hline Avg. & $0.00 / 0.40 / 2.06 / 0$ & $-10.00 / 1.00 / 18.18 / 15$ & $0.00 / 0.91 / 4.86 / 17$ & $-2.85 / 0.03 / 1.72 / 0$ & $0.00 / 0.40 / 2.06 / 0$ \\
\hline \multicolumn{6}{|l|}{ TNV25 } \\
\hline D1 & $0.00 / 0.24 / 1.18 / 0$ & $-10.00 /-1.45 / 9.09 / 1$ & $0.00 / 0.46 / 2.08 / 0$ & $-0.97 / 0.08 / 0.92 / 0$ & $0.00 / 0.24 / 1.18 / 0$ \\
\hline D2 & $0.00 / 0.26 / 1.71 / 0$ & $0.00 / 0.57 / 10.00 / 3$ & $0.00 / 0.45 / 2.82 / 0$ & $-0.08 / 0.13 / 1.31 / 0$ & $0.00 / 0.26 / 1.71 / 0$ \\
\hline D3 & $0.00 / 0.03 / 0.19 / 0$ & $0.00 / 0.00 / 0.00 / 0$ & $0.00 / 0.06 / 0.64 / 0$ & $-0.21 / 0.00 / 0.20 / 0$ & $0.00 / 0.03 / 0.19 / 0$ \\
\hline D4 & $0.00 / 0.00 / 0.00 / 0$ & $0.00 / 0.00 / 0.00 / 0$ & $0.00 / 0.00 / 0.00 / 0$ & $0.00 / 0.00 / 0.00 / 0$ & $0.00 / 0.00 / 0.00 / 0$ \\
\hline Avg. & $0.00 / 0.21 / 1.71 / 0$ & $-10.00 /-0.28 / 10.00 / 4$ & $0.00 / 0.37 / 2.82 / 0$ & $-0.97 / 0.09 / 1.31 / 0$ & $0.00 / 0.21 / 1.71 / 0$ \\
\hline \multicolumn{6}{|l|}{ TNV50 } \\
\hline D1 & $0.00 / 0.35 / 1.42 / 0$ & $-10.00 / 0.16 / 9.09 / 3$ & $-1.15 / 0.66 / 2.56 / 0$ & $-1.12 / 0.13 / 2.01 / 0$ & $0.00 / 0.35 / 1.42 / 0$ \\
\hline D2 & $0.00 / 0.34 / 1.71 / 0$ & $0.00 / 0.57 / 10.00 / 3$ & $0.00 / 0.71 / 2.82 / 0$ & $-1.66 / 0.08 / 1.31 / 0$ & $0.00 / 0.34 / 1.71 / 0$ \\
\hline D3 & $0.00 / 0.28 / 2.01 / 0$ & $0.00 / 1.02 / 7.14 / 2$ & $0.00 / 0.41 / 3.14 / 1$ & $0.00 / 0.18 / 1.45 / 0$ & $0.00 / 0.28 / 2.01 / 0$ \\
\hline D4 & $0.00 / 0.00 / 0.00 / 0$ & $0.00 / 0.00 / 0.00 / 0$ & $0.00 / 0.00 / 0.00 / 0$ & $0.00 / 0.00 / 0.00 / 0$ & $0.00 / 0.00 / 0.00 / 0$ \\
\hline Avg. & $0.00 / 0.32 / 2.01 / 0$ & $-10.00 / 0.43 / 10.00 / 8$ & $-1.15 / 0.60 / 3.14 / 1$ & $-1.66 / 0.11 / 2.01 / 0$ & $0.00 / 0.32 / 2.01 / 0$ \\
\hline \multicolumn{6}{|l|}{ TNV75 } \\
\hline D1 & $0.00 / 0.36 / 1.42 / 0$ & $-10.00 / 0.16 / 9.09 / 3$ & $-1.15 / 0.67 / 2.56 / 0$ & $-1.12 / 0.13 / 2.01 / 0$ & $0.00 / 0.36 / 1.42 / 0$ \\
\hline D2 & $0.00 / 0.42 / 1.94 / 0$ & $0.00 / 0.75 / 18.18 / 3$ & $0.00 / 1.01 / 4.86 / 7$ & $-2.34 /-0.01 / 0.95 / 0$ & $0.00 / 0.42 / 1.94 / 0$ \\
\hline D3 & $0.00 / 0.79 / 2.01 / 0$ & $0.00 / 3.06 / 7.14 / 6$ & $0.00 / 1.23 / 3.14 / 2$ & $0.00 / 0.43 / 1.45 / 0$ & $0.00 / 0.79 / 2.01 / 0$ \\
\hline D4 & $0.00 / 0.00 / 0.00 / 0$ & $0.00 / 0.00 / 0.00 / 0$ & $0.00 / 0.00 / 0.00 / 0$ & $0.00 / 0.00 / 0.00 / 0$ & $0.00 / 0.00 / 0.00 / 0$ \\
\hline Avg. & $0.00 / 0.41 / 2.01 / 0$ & $-10.00 / 0.76 / 18.18 / 12$ & $-1.15 / 0.84 / 4.86 / 9$ & $-2.34 / 0.10 / 2.01 / 0$ & $0.00 / 0.41 / 2.01 / 0$ \\
\hline
\end{tabular}


Table EC.6 Objective III - Relative savings obtained with the different distribution policies compared to VRPTW, grouped by demand scenario

\begin{tabular}{|c|c|c|c|c|c|}
\hline \multirow[b]{2}{*}{$\begin{array}{l}\text { Policy/ } \\
\text { Demand } \\
\text { scenario }\end{array}$} & \multicolumn{5}{|c|}{ Min./Avg./Max. \% Savings/\# Instances with savings $>3 \%$ in } \\
\hline & Objective value & Number of routes & $\begin{array}{c}\text { Variable routing } \\
\text { costs }\end{array}$ & Route durations & $\begin{array}{l}\text { Sum of variable } \\
\text { routing costs and } \\
\text { costs related to } \\
\text { route durations }\end{array}$ \\
\hline \multicolumn{6}{|l|}{ SDVRPTW } \\
\hline D1 & $0.00 / 0.22 / 9.07 / 1$ & $0.00 / 0.22 / 9.09 / 1$ & $-2.50 / 1.87 / 11.78 / 15$ & $-2.51 / 0.70 / 17.71 / 2$ & $-2.50 / 1.18 / 15.76 / 3$ \\
\hline D2 & $0.00 / 0.00 / 0.01 / 0$ & $0.00 / 0.00 / 0.00 / 0$ & $0.00 / 1.30 / 9.37 / 9$ & $-1.66 / 0.05 / 1.41 / 0$ & $0.00 / 0.49 / 3.12 / 1$ \\
\hline D3 & $0.00 / 0.04 / 0.19 / 0$ & $0.00 / 0.00 / 0.00 / 0$ & $0.00 / 8.90 / 12.71 / 12$ & $-0.67 / 10.86 / 40.57 / 12$ & $0.00 / 10.98 / 36.72 / 12$ \\
\hline D4 & $0.00 / 0.01 / 0.10 / 0$ & $0.00 / 0.00 / 0.00 / 0$ & $0.00 / 1.29 / 8.48 / 1$ & $-0.20 / 2.53 / 20.48 / 1$ & $0.00 / 2.36 / 18.78 / 1$ \\
\hline Avg. & $0.00 / 0.09 / 9.07 / 1$ & $0.00 / 0.08 / 9.09 / 1$ & $-2.50 / 2.43 / 12.71 / 37$ & $-2.51 / 1.77 / 40.57 / 15$ & $-2.50 / 2.15 / 36.72 / 17$ \\
\hline \multicolumn{6}{|l|}{ NV2 } \\
\hline D1 & $0.00 / 0.22 / 9.07 / 1$ & $0.00 / 0.22 / 9.09 / 1$ & $-2.50 / 1.87 / 11.78 / 15$ & $-2.51 / 0.70 / 17.71 / 2$ & $-2.50 / 1.18 / 15.76 / 3$ \\
\hline D2 & $0.00 / 0.00 / 0.01 / 0$ & $0.00 / 0.00 / 0.00 / 0$ & $0.00 / 1.30 / 9.37 / 9$ & $-1.66 / 0.05 / 1.41 / 0$ & $0.00 / 0.49 / 3.12 / 1$ \\
\hline D3 & $0.00 / 0.04 / 0.19 / 0$ & $0.00 / 0.00 / 0.00 / 0$ & $0.00 / 8.87 / 12.71 / 12$ & $-0.67 / 10.88 / 40.57 / 12$ & $0.00 / 10.98 / 36.72 / 12$ \\
\hline D4 & $0.00 / 0.01 / 0.10 / 0$ & $0.00 / 0.00 / 0.00 / 0$ & $0.00 / 1.29 / 8.48 / 1$ & $-0.20 / 2.53 / 20.48 / 1$ & $0.00 / 2.36 / 18.78 / 1$ \\
\hline Avg. & $0.00 / 0.09 / 9.07 / 1$ & $0.00 / 0.08 / 9.09 / 1$ & $-2.50 / 2.43 / 12.71 / 37$ & $-2.51 / 1.78 / 40.57 / 15$ & $-2.50 / 2.15 / 36.72 / 17$ \\
\hline \multicolumn{6}{|l|}{ So } \\
\hline D1 & $0.00 / 0.22 / 9.07 / 1$ & $0.00 / 0.22 / 9.09 / 1$ & $-2.50 / 1.72 / 11.78 / 13$ & $-3.39 / 0.62 / 17.71 / 2$ & $-3.03 / 1.07 / 15.76 / 3$ \\
\hline D2 & $0.00 / 0.00 / 0.01 / 0$ & $0.00 / 0.00 / 0.00 / 0$ & $0.00 / 1.16 / 9.37 / 8$ & $-1.66 / 0.01 / 0.95 / 0$ & $0.00 / 0.42 / 2.83 / 0$ \\
\hline D3 & $0.00 / 0.04 / 0.19 / 0$ & $0.00 / 0.00 / 0.00 / 0$ & $0.00 / 8.83 / 12.71 / 12$ & $-0.71 / 10.81 / 40.17 / 12$ & $0.00 / 10.92 / 36.41 / 12$ \\
\hline D4 & $0.00 / 0.01 / 0.09 / 0$ & $0.00 / 0.00 / 0.00 / 0$ & $0.00 / 1.29 / 8.48 / 1$ & $-0.20 / 2.07 / 16.79 / 1$ & $0.00 / 1.97 / 15.62 / 1$ \\
\hline Avg. & $0.00 / 0.09 / 9.07 / 1$ & $0.00 / 0.08 / 9.09 / 1$ & $-2.50 / 2.31 / 12.71 / 34$ & $-3.39 / 1.69 / 40.17 / 15$ & $-3.03 / 2.04 / 36.41 / 16$ \\
\hline \multicolumn{6}{|l|}{ TNV25 } \\
\hline D1 & $0.00 / 0.22 / 9.07 / 1$ & $0.00 / 0.22 / 9.09 / 1$ & $-4.15 / 0.22 / 1.91 / 0$ & $-2.41 / 0.04 / 2.03 / 0$ & $-3.11 / 0.12 / 1.54 / 0$ \\
\hline D2 & $0.00 / 0.00 / 0.01 / 0$ & $0.00 / 0.00 / 0.00 / 0$ & $0.00 / 0.54 / 4.47 / 1$ & $0.00 / 0.16 / 1.58 / 0$ & $0.00 / 0.32 / 2.79 / 0$ \\
\hline D3 & $0.00 / 0.02 / 0.18 / 0$ & $0.00 / 0.00 / 0.00 / 0$ & $0.00 / 3.40 / 12.30 / 7$ & $0.00 / 4.54 / 37.93 / 7$ & $0.00 / 4.60 / 34.43 / 7$ \\
\hline D4 & $0.00 / 0.01 / 0.05 / 0$ & $0.00 / 0.00 / 0.00 / 0$ & $0.00 / 0.33 / 2.62 / 0$ & $0.00 / 1.38 / 11.02 / 1$ & $0.00 / 1.23 / 9.83 / 1$ \\
\hline Avg. & $0.00 / 0.08 / 9.07 / 1$ & $0.00 / 0.08 / 9.09 / 1$ & $-4.15 / 0.76 / 12.30 / 8$ & $-2.41 / 0.74 / 37.93 / 8$ & $-3.11 / 0.83 / 34.43 / 8$ \\
\hline \multicolumn{6}{|l|}{ TNV50 } \\
\hline D1 & $0.00 / 0.22 / 9.07 / 1$ & $0.00 / 0.22 / 9.09 / 1$ & $-4.15 / 1.42 / 11.78 / 4$ & $-2.41 / 0.72 / 17.71 / 2$ & $-3.11 / 1.00 / 15.76 / 3$ \\
\hline D2 & $0.00 / 0.00 / 0.01 / 0$ & $0.00 / 0.00 / 0.00 / 0$ & $0.00 / 1.05 / 9.37 / 3$ & $-1.66 / 0.09 / 1.58 / 0$ & $0.00 / 0.42 / 2.79 / 0$ \\
\hline D3 & $0.00 / 0.04 / 0.18 / 0$ & $0.00 / 0.00 / 0.00 / 0$ & $0.00 / 8.10 / 13.72 / 12$ & $-0.67 / 10.32 / 39.08 / 12$ & $0.00 / 10.26 / 35.61 / 12$ \\
\hline D4 & $0.00 / 0.01 / 0.05 / 0$ & $0.00 / 0.00 / 0.00 / 0$ & $0.00 / 0.56 / 2.62 / 0$ & $-0.20 / 1.35 / 11.02 / 1$ & $0.00 / 1.24 / 9.83 / 1$ \\
\hline Avg. & $0.00 / 0.09 / 9.07 / 1$ & $0.00 / 0.08 / 9.09 / 1$ & $-4.15 / 2.01 / 13.72 / 19$ & $-2.41 / 1.65 / 39.08 / 15$ & $-3.11 / 1.89 / 35.61 / 16$ \\
\hline \multicolumn{6}{|l|}{ TNV75 } \\
\hline D1 & $0.00 / 0.22 / 9.07 / 1$ & $0.00 / 0.22 / 9.09 / 1$ & $-2.50 / 1.47 / 11.78 / 4$ & $-2.51 / 0.72 / 17.71 / 2$ & $-2.50 / 1.01 / 15.76 / 3$ \\
\hline D2 & $0.00 / 0.00 / 0.01 / 0$ & $0.00 / 0.00 / 0.00 / 0$ & $0.00 / 1.30 / 9.37 / 9$ & $-1.66 / 0.05 / 1.41 / 0$ & $0.00 / 0.49 / 3.12 / 1$ \\
\hline D3 & $0.00 / 0.04 / 0.18 / 0$ & $0.00 / 0.00 / 0.00 / 0$ & $0.00 / 8.93 / 13.72 / 12$ & $-0.67 / 10.80 / 39.08 / 12$ & $0.00 / 10.90 / 35.61 / 12$ \\
\hline D4 & $0.00 / 0.01 / 0.10 / 0$ & $0.00 / 0.00 / 0.00 / 0$ & $0.00 / 1.29 / 8.48 / 1$ & $-0.20 / 2.53 / 20.48 / 1$ & $0.00 / 2.36 / 18.78 / 1$ \\
\hline Avg. & $0.00 / 0.09 / 9.07 / 1$ & $0.00 / 0.08 / 9.09 / 1$ & $-2.50 / 2.29 / 13.72 / 26$ & $-2.51 / 1.77 / 39.08 / 15$ & $-2.50 / 2.08 / 35.61 / 17$ \\
\hline
\end{tabular}


Table EC.7 Impact of inconvenience constraints on algorithm performance for objective I, grouped by demand

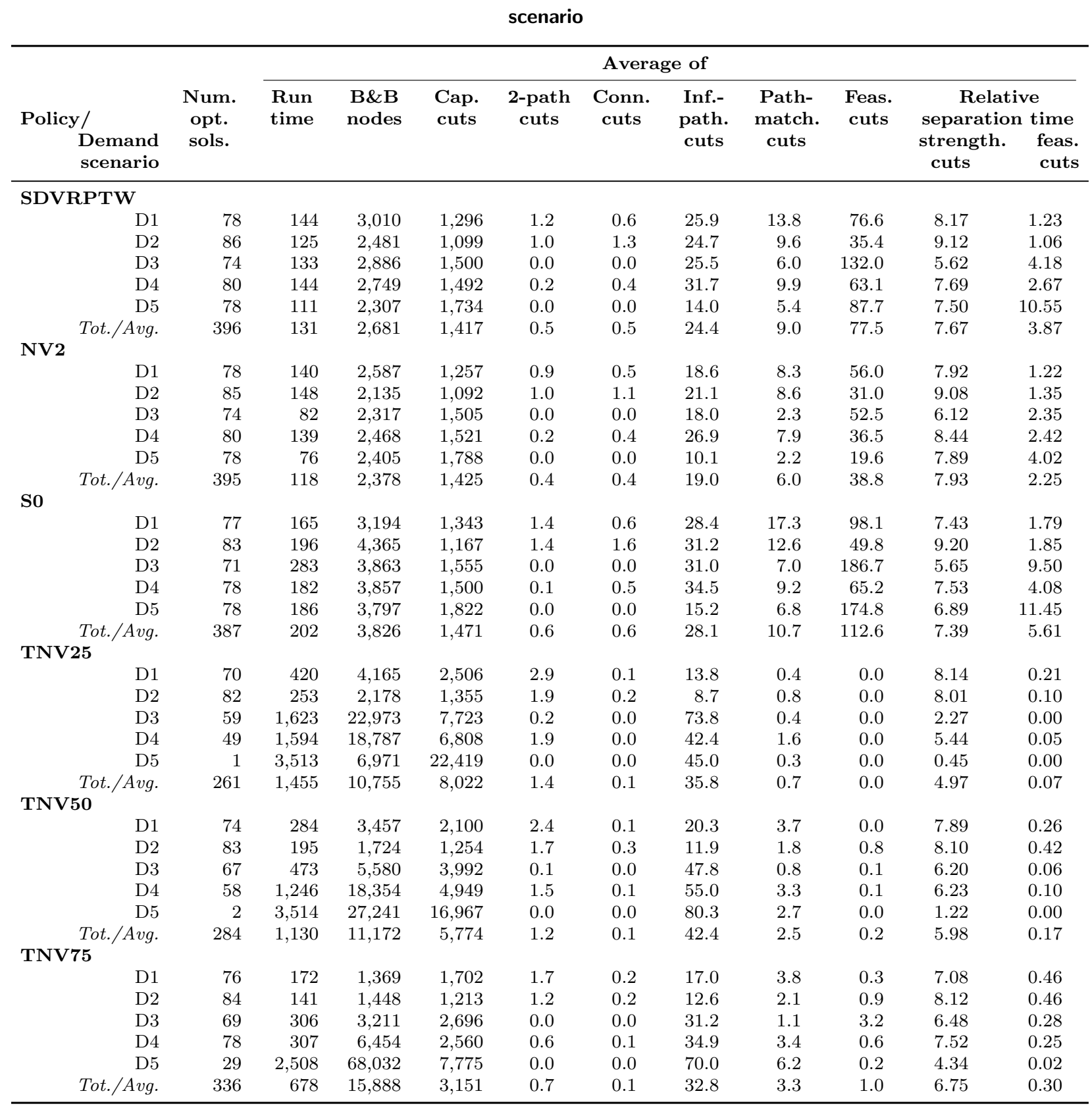


Table EC.8 Impact of inconvenience constraints on algorithm performance for objective II, grouped by demand

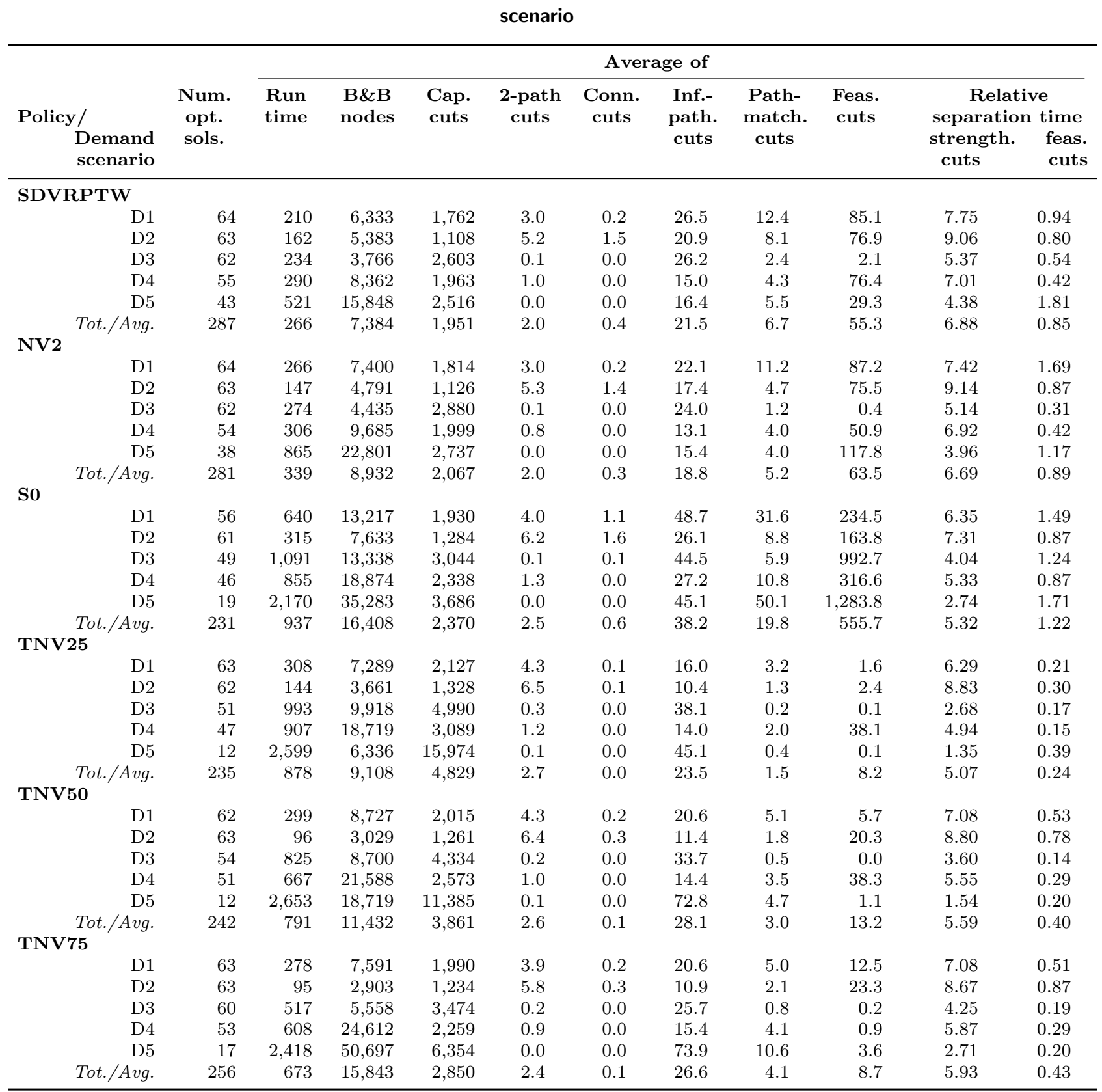


Table EC.9 Impact of inconvenience constraints on algorithm performance for objective III, grouped by demand scenario

\begin{tabular}{|c|c|c|c|c|c|c|c|c|c|c|c|}
\hline \multirow[b]{2}{*}{$\begin{array}{r}\text { Policy / } \\
\text { Demand } \\
\text { scenario }\end{array}$} & \multirow[b]{2}{*}{$\begin{array}{l}\text { Num. } \\
\text { opt. } \\
\text { sols. }\end{array}$} & \multicolumn{10}{|c|}{ Average of } \\
\hline & & $\begin{array}{l}\text { Run } \\
\text { time }\end{array}$ & $\begin{array}{c}\text { B\&B } \\
\text { nodes }\end{array}$ & $\begin{array}{l}\text { Cap. } \\
\text { cuts }\end{array}$ & $\begin{array}{l}\text { 2-path } \\
\text { cuts }\end{array}$ & $\begin{array}{c}\text { Conn. } \\
\text { cuts }\end{array}$ & $\begin{array}{l}\text { Inf.- } \\
\text { path. } \\
\text { cuts }\end{array}$ & $\begin{array}{l}\text { Path- } \\
\text { match. } \\
\text { cuts }\end{array}$ & $\begin{array}{c}\text { Feas. } \\
\text { cuts }\end{array}$ & $\begin{array}{r}\text { Re } \\
\text { separa } \\
\text { strengt } \\
\text { cuts }\end{array}$ & $\begin{array}{l}\text { ive } \\
\text { n time } \\
\text { feas. } \\
\text { cuts }\end{array}$ \\
\hline \multicolumn{12}{|l|}{ SDVRPTW } \\
\hline D1 & 64 & 256 & 7,196 & 1,929 & 3.4 & 0.3 & 34.5 & 20.4 & 153.5 & 7.95 & 1.25 \\
\hline D2 & 57 & 188 & 4,827 & 1,347 & 4.5 & 1.3 & 28.7 & 8.9 & 59.0 & 9.64 & 0.91 \\
\hline D3 & 56 & 312 & 5,461 & 2,500 & 0.1 & 0.0 & 32.5 & 7.1 & 10.3 & 4.94 & 8.90 \\
\hline $\mathrm{D} 4$ & 45 & 314 & 12,406 & 2,117 & 1.5 & 0.0 & 39.7 & 18.7 & 179.3 & 6.49 & 2.21 \\
\hline D5 & 32 & 949 & 26,907 & 3,062 & 0.0 & 0.0 & 21.2 & 20.0 & 34.4 & 3.82 & 11.60 \\
\hline Tot./Avg. & 254 & 351 & 9,688 & 2,100 & 2.2 & 0.4 & 32.0 & 14.5 & 90.3 & 6.89 & 4.33 \\
\hline \multicolumn{12}{|l|}{ NV2 } \\
\hline D1 & 63 & 317 & 9,062 & 2,084 & 3.4 & 0.3 & 30.0 & 16.0 & 158.7 & 8.13 & 2.34 \\
\hline D2 & 57 & 184 & 4,201 & 1,348 & 4.3 & 1.1 & 19.8 & 5.9 & 49.4 & 9.34 & 2.46 \\
\hline D3 & 56 & 256 & 5,425 & 2,732 & 0.0 & 0.0 & 31.9 & 4.6 & 8.7 & 4.92 & 2.83 \\
\hline $\mathrm{D} 4$ & 43 & 584 & 16,083 & 2,734 & 1.8 & 0.1 & 35.8 & 13.7 & 205.5 & 6.27 & 3.32 \\
\hline D5 & 26 & 1,280 & 30,123 & 3,497 & 0.0 & 0.0 & 16.7 & 12.2 & 11.0 & 3.72 & 3.77 \\
\hline Tot./Avg. & 245 & 442 & 11,067 & 2,355 & 2.1 & 0.3 & 27.5 & 10.3 & 90.8 & 6.81 & 2.83 \\
\hline \multicolumn{12}{|l|}{ So } \\
\hline D1 & 52 & 990 & 20,676 & 2,386 & 4.9 & 1.7 & 79.5 & 59.3 & 475.2 & 6.34 & 1.29 \\
\hline D2 & 54 & 356 & 9,107 & 1,436 & 5.0 & 2.2 & 43.6 & 15.7 & 203.2 & 7.14 & 0.73 \\
\hline D3 & 39 & 1,356 & 18,140 & 2,999 & 0.1 & 0.1 & 68.6 & 17.3 & 822.0 & 3.78 & 8.83 \\
\hline $\mathrm{D} 4$ & 31 & 1,568 & 26,968 & 3,174 & 2.2 & 1.1 & 126.2 & 81.6 & 608.9 & 4.14 & 9.87 \\
\hline D5 & 11 & 2,626 & 36,924 & 4,108 & 0.0 & 0.0 & 51.4 & 76.0 & $1,193.5$ & 2.27 & 16.35 \\
\hline Tot./Avg. & 187 & 1,237 & 20,683 & 2,664 & 2.8 & 1.1 & 73.8 & 46.3 & 604.8 & 5.06 & 6.25 \\
\hline \multicolumn{12}{|l|}{ TNV25 } \\
\hline D1 & 58 & 768 & 14,615 & 3,674 & 6.3 & 0.1 & 26.1 & 5.1 & 1.5 & 6.00 & 0.10 \\
\hline D2 & 57 & 130 & 2,760 & 1,727 & 5.8 & 0.1 & 12.1 & 1.6 & 2.4 & 8.08 & 0.37 \\
\hline D3 & 18 & 2,528 & 11,604 & 12,606 & 0.5 & 0.0 & 50.6 & 0.6 & 0.3 & 1.20 & 0.10 \\
\hline D4 & 11 & 2,872 & 16,488 & 18,311 & 4.6 & 0.0 & 68.8 & 7.8 & 6.4 & 1.50 & 0.03 \\
\hline D5 & 1 & 3,441 & 5,597 & 26,429 & 0.0 & 0.0 & 17.1 & 1.0 & 0.1 & 0.24 & 0.00 \\
\hline Tot./Avg. & 145 & 1,722 & 10,486 & 10,666 & 3.8 & 0.0 & 34.8 & 3.3 & 2.1 & 3.90 & 0.14 \\
\hline \multicolumn{12}{|l|}{ TNV50 } \\
\hline D1 & 62 & 520 & 11,530 & 2,956 & 5.9 & 0.1 & 31.3 & 9.1 & 30.4 & 6.70 & 0.30 \\
\hline D2 & 56 & 168 & 3,207 & 1,649 & 6.1 & 0.3 & 15.7 & 2.5 & 6.7 & 8.05 & 0.30 \\
\hline D3 & 27 & 2,082 & 10,350 & 11,919 & 0.4 & 0.0 & 54.9 & 1.7 & 0.6 & 2.20 & 0.06 \\
\hline D4 & 11 & 2,857 & 21,191 & 18,168 & 5.4 & 0.0 & 100.6 & 18.4 & 82.7 & 1.68 & 0.04 \\
\hline D5 & 0 & 3,488 & 10,455 & 27,548 & 0.0 & 0.0 & 65.4 & 7.2 & 1.1 & 0.41 & 0.00 \\
\hline Tot./Avg. & 156 & 1,573 & 10,978 & 10,432 & 3.9 & 0.1 & 49.6 & 7.4 & 24.1 & 4.35 & 0.16 \\
\hline \multicolumn{12}{|l|}{ TNV75 } \\
\hline D1 & 64 & 413 & 10,199 & 2,748 & 5.7 & 0.2 & 31.0 & 9.1 & 39.4 & 6.87 & 0.27 \\
\hline D2 & 56 & 163 & 3,231 & 1,592 & 5.6 & 0.5 & 16.2 & 3.7 & 11.7 & 8.26 & 0.53 \\
\hline D3 & 32 & 1,636 & 10,083 & 8,618 & 0.3 & 0.0 & 53.8 & 2.5 & 1.2 & 2.82 & 0.17 \\
\hline $\mathrm{D} 4$ & 30 & 1,650 & 25,655 & 7,728 & 2.9 & 0.0 & 70.9 & 13.5 & 27.6 & 3.40 & 0.22 \\
\hline D5 & 1 & 3,454 & 32,149 & 19,626 & 0.0 & 0.0 & 72.4 & 26.8 & 6.7 & 0.98 & 0.00 \\
\hline Tot./Avg. & 183 & 1,229 & 14,114 & 6,791 & 3.3 & 0.2 & 45.0 & 9.4 & 18.5 & 4.95 & 0.27 \\
\hline
\end{tabular}




\section{EC.3. Results grouped by Solomon Class}

Tables EC.10-EC.18 provide further details on the aggregated results given in Tables 4, 5, and 6:

Tables EC.10-EC.12 show the effects of the different objective functions on the solution structure of the different SDVRPTW policies compared to the VRPTW; Tables EC.13-EC.15 indicate the relative savings obtained with the different objective functions.

Similar to the case of the demand scenarios, the difficulty of the instances we constructed also varies strongly between the different Solomon classes. The 115 instances solved to optimality with all objective functions are divided between the classes as follows:

$\begin{array}{lr}\mathrm{C} 1 & 27 \\ \mathrm{C} 2 & 21 \\ \mathrm{R} 1 & 22 \\ \mathrm{R} 2 & 9 \\ \mathrm{RC} 1 & 25 \\ \mathrm{RC} 2 & 11\end{array}$

As can be seen, the instances derived from classes R2 and RC2, i.e., the random as well as the random and clustered instances with wide time windows, are on average much harder to solve than those from other classes.

Tables EC.16-EC.18 describe the impact of inconvenience constraints on the performance of the branch-and-cut algorithm. Again, the results given in these tables were obtained using, for each objective, the instances that were solved to optimality with the SDVRPTW policy for this objective.

The following observations can be made in the tables:

- For objective I, the number of visits per customer and the percentage of split customers is lowest for the RC instances.

- For objective II, there is no split customer for the C instances (there were several instances with split customers, but these were not solved to optimality for all policies).

- For objective III, the number of visits per customer and the percentage of split customers is lower for the $\mathrm{C}$ and for the RC instances than for the $\mathrm{R}$ instances.

- The percentage of split customers with deliveries fully synchronized is lowest for the R instances.

- In general, the timespan between the first and last delivery in relation to the time window width is highest for the $\mathrm{R} 1$ instances.

- For objective I, the savings in the objective function are highest for the C2 instances, as are the savings in the number of routes.

- For objective II, both types of savings are highest for the R instances.

- For objective III, the savings in the objective function are highest for the C2 and R1 instances; savings in the number of routes are obtained only for R1 instances. 
- Solomon classes can be ranked by decreasing savings as follows: C, R, and RC for objective I; $\mathrm{R}, \mathrm{RC}$, and $\mathrm{C}$ for objective II; R1 and $\mathrm{C} 2$ for objective III.

- The larger the average number of separated feasibility cuts, the harder to obtain integer feasibility. This number is largest in classes $\mathrm{C}$ and RC for objective I, and in classes R and RC for objectives II and III. In particular, for classes R2 and RC2 with objectives II and III, the average number of separated feasibility cuts is mostly larger than for the other classes.

We may conclude that the effects of the geographical distribution of customers depend on the objective function. 
Table EC.10 Objective I - Effect of the different distribution policies on solution structure compared to VRPTW, grouped by Solomon class

\begin{tabular}{|c|c|c|c|c|c|}
\hline \multirow[b]{2}{*}{$\begin{array}{r}\text { Policy / } \\
\text { Solomon } \\
\text { class }\end{array}$} & \multicolumn{5}{|c|}{ Average of } \\
\hline & $\begin{array}{l}\text { Number of } \\
\text { visits per } \\
\text { customer }\end{array}$ & $\begin{array}{l}\text { Percentage of } \\
\text { split customers }\end{array}$ & $\begin{array}{l}\text { Number of } \\
\text { visits per split } \\
\text { customer }\end{array}$ & $\begin{array}{c}\text { Percentage of } \\
\text { split customers } \\
\text { with deliveries } \\
\text { fully } \\
\text { synchronized }\end{array}$ & $\begin{array}{c}\text { Timespan } \\
\text { between first and } \\
\text { last delivery in } \\
\text { relation to time } \\
\text { window width in } \\
\%\end{array}$ \\
\hline \multicolumn{6}{|l|}{ SDVRPTW } \\
\hline $\mathrm{C} 1$ & 1.09 & 9.33 & 2.00 & 35.99 & 26.29 \\
\hline $\mathrm{C} 2$ & 1.13 & 13.14 & 2.00 & 25.34 & 31.28 \\
\hline $\mathrm{R} 1$ & 1.12 & 11.64 & 2.00 & 5.95 & 40.08 \\
\hline $\mathrm{R} 2$ & 1.12 & 11.56 & 2.00 & 2.78 & 25.09 \\
\hline $\mathrm{RC} 1$ & 1.07 & 7.20 & 2.00 & 19.91 & 26.94 \\
\hline $\mathrm{RC} 2$ & 1.06 & 5.82 & 2.00 & 20.83 & 21.58 \\
\hline Avg. & 1.10 & 9.84 & 2.00 & 20.95 & 29.44 \\
\hline \multicolumn{6}{|l|}{ NV2 } \\
\hline $\mathrm{C} 2$ & 1.13 & 12.95 & 2.00 & 25.45 & 31.22 \\
\hline $\mathrm{R} 1$ & 1.12 & 11.82 & 2.00 & 5.95 & 37.60 \\
\hline $\mathrm{R} 2$ & 1.13 & 12.89 & 2.00 & 2.78 & 28.40 \\
\hline $\mathrm{RC} 1$ & 1.07 & 7.12 & 2.00 & 15.74 & 26.42 \\
\hline $\mathrm{RC} 2$ & 1.06 & 6.00 & 2.00 & 29.17 & 22.90 \\
\hline Avg. & 1.10 & 9.98 & 2.00 & 21.37 & 28.37 \\
\hline \multicolumn{6}{|l|}{ So } \\
\hline $\mathrm{C} 1$ & 1.10 & 9.48 & 2.00 & 100.00 & 0.00 \\
\hline $\mathrm{C} 2$ & 1.13 & 13.33 & 2.00 & 100.00 & 0.00 \\
\hline $\mathrm{R} 1$ & 1.11 & 11.09 & 2.00 & 100.00 & 0.00 \\
\hline $\mathrm{R} 2$ & 1.12 & 12.00 & 2.00 & 100.00 & 0.00 \\
\hline $\mathrm{RC} 1$ & 1.07 & 7.12 & 2.00 & 100.00 & 0.00 \\
\hline $\mathrm{RC} 2$ & 1.06 & 6.18 & 2.00 & 100.00 & 0.00 \\
\hline Avg. & 1.10 & 9.86 & 2.00 & 100.00 & 0.00 \\
\hline \multicolumn{6}{|l|}{ TNV25 } \\
\hline $\mathrm{C} 1$ & 1.03 & 2.81 & 2.00 & 42.11 & 26.49 \\
\hline $\mathrm{C} 2$ & 1.04 & 3.81 & 2.00 & 40.00 & 11.96 \\
\hline $\mathrm{R} 1$ & 1.04 & 3.45 & 2.00 & 15.79 & 20.46 \\
\hline $\mathrm{R} 2$ & 1.04 & 4.00 & 2.00 & 11.11 & 19.21 \\
\hline $\mathrm{RC} 1$ & 1.02 & 2.40 & 2.00 & 0.00 & 35.40 \\
\hline $\mathrm{RC} 2$ & 1.02 & 2.18 & 2.00 & 14.29 & 27.15 \\
\hline Avg. & 1.03 & 3.06 & 2.00 & 22.09 & 24.11 \\
\hline \multicolumn{6}{|l|}{ TNV50 } \\
\hline $\mathrm{C} 1$ & 1.06 & 5.78 & 2.00 & 35.19 & 36.66 \\
\hline $\mathrm{C} 2$ & 1.08 & 7.62 & 2.00 & 25.00 & 24.48 \\
\hline $\mathrm{R} 1$ & 1.07 & 6.55 & 2.00 & 11.90 & 33.82 \\
\hline $\mathrm{R} 2$ & 1.06 & 6.22 & 2.00 & 11.11 & 23.34 \\
\hline $\mathrm{RC} 1$ & 1.04 & 3.36 & 2.11 & 11.11 & 32.47 \\
\hline $\mathrm{RC} 2$ & 1.03 & 3.27 & 2.00 & 31.25 & 22.67 \\
\hline Avg. & 1.06 & 5.53 & 2.02 & 21.88 & 30.60 \\
\hline \multicolumn{6}{|l|}{ TNV75 } \\
\hline $\mathrm{C} 1$ & 1.08 & 7.85 & 2.00 & 38.58 & 22.83 \\
\hline $\mathrm{C} 2$ & 1.10 & 9.71 & 2.00 & 27.94 & 26.05 \\
\hline $\mathrm{R} 1$ & 1.09 & 8.91 & 2.00 & 7.14 & 38.74 \\
\hline $\mathrm{R} 2$ & 1.09 & 9.33 & 2.00 & 0.00 & 29.40 \\
\hline $\mathrm{RC} 1$ & 1.06 & 6.00 & 2.00 & 11.57 & 28.94 \\
\hline $\mathrm{RC} 2$ & 1.05 & 5.09 & 2.00 & 29.17 & 21.42 \\
\hline Avg. & 1.08 & 7.84 & 2.00 & 21.35 & 28.17 \\
\hline
\end{tabular}


Table EC.11 Objective II - Effect of the different distribution policies on solution structure compared to VRPTW, grouped by Solomon class

\begin{tabular}{|c|c|c|c|c|c|}
\hline \multirow[b]{2}{*}{$\begin{array}{r}\text { Policy / } \\
\text { Solomon } \\
\text { class }\end{array}$} & \multicolumn{5}{|c|}{ Average of } \\
\hline & $\begin{array}{l}\text { Number of } \\
\text { visits per } \\
\text { customer }\end{array}$ & $\begin{array}{c}\text { Percentage of } \\
\text { split customers }\end{array}$ & $\begin{array}{c}\text { Number of } \\
\text { visits per split } \\
\text { customer }\end{array}$ & $\begin{array}{c}\text { Percentage of } \\
\text { split customers } \\
\text { with deliveries } \\
\text { fully } \\
\text { synchronized }\end{array}$ & $\begin{array}{c}\text { Timespan } \\
\text { between first and } \\
\text { last delivery in } \\
\text { relation to time } \\
\text { window width in } \\
\%\end{array}$ \\
\hline \multicolumn{6}{|l|}{ SDVRPTW } \\
\hline $\mathrm{C} 1$ & 1.00 & 0.00 & n.a. & n.a. & n.a. \\
\hline $\mathrm{C} 2$ & 1.00 & 0.00 & n.a. & n.a. & $n . a$. \\
\hline $\mathrm{R} 1$ & 1.08 & 8.18 & 2.00 & 12.70 & 30.19 \\
\hline $\mathrm{R} 2$ & 1.08 & 8.44 & 2.00 & 3.70 & 31.36 \\
\hline $\mathrm{RC} 1$ & 1.03 & 2.88 & 2.04 & 8.33 & 21.26 \\
\hline $\mathrm{RC} 2$ & 1.02 & 1.45 & 2.00 & 16.67 & 9.51 \\
\hline Avg. & 1.03 & 2.99 & 2.01 & 10.00 & 13.76 \\
\hline \multicolumn{6}{|l|}{ NV2 } \\
\hline C1 & 1.00 & 0.00 & n.a. & n.a. & $n . a$. \\
\hline $\mathrm{C} 2$ & 1.00 & 0.00 & n.a. & n.a. & n.a. \\
\hline R1 & 1.08 & 8.18 & 2.00 & 12.70 & 28.94 \\
\hline $\mathrm{R} 2$ & 1.08 & 8.44 & 2.00 & 3.70 & 35.01 \\
\hline $\mathrm{RC} 1$ & 1.03 & 2.88 & 2.00 & 11.11 & 23.69 \\
\hline $\mathrm{RC} 2$ & 1.02 & 1.45 & 2.00 & 16.67 & 8.23 \\
\hline Avg. & 1.03 & 2.99 & 2.00 & 10.74 & 14.21 \\
\hline \multicolumn{6}{|l|}{ So } \\
\hline C1 & 1.00 & 0.00 & $n . a$. & n.a. & n.a. \\
\hline $\mathrm{C} 2$ & 1.00 & 0.00 & n.a. & $n . a$. & n.a. \\
\hline R1 & 1.07 & 6.73 & 2.00 & 100.00 & 0.00 \\
\hline $\mathrm{R} 2$ & 1.08 & 7.56 & 2.00 & 100.00 & 0.00 \\
\hline $\mathrm{RC} 1$ & 1.02 & 1.92 & 2.07 & 100.00 & 0.00 \\
\hline $\mathrm{RC} 2$ & 1.01 & 1.09 & 2.00 & 100.00 & 0.00 \\
\hline Avg. & 1.02 & 2.40 & 2.01 & 100.00 & 0.00 \\
\hline \multicolumn{6}{|l|}{ TNV25 } \\
\hline $\mathrm{C} 1$ & 1.00 & 0.00 & $n . a$. & $n . a$. & n.a. \\
\hline $\mathrm{C} 2$ & 1.00 & 0.00 & n.a. & n.a. & n.a. \\
\hline R1 & 1.03 & 2.91 & 2.00 & 12.50 & 30.68 \\
\hline $\mathrm{R} 2$ & 1.03 & 3.11 & 2.00 & 0.00 & 30.90 \\
\hline $\mathrm{RC} 1$ & 1.00 & 0.40 & 2.00 & 66.67 & 3.03 \\
\hline $\mathrm{RC} 2$ & 1.00 & 0.00 & n.a. & $n . a$. & $n . a$. \\
\hline Avg. & 1.01 & 0.89 & 2.00 & 15.38 & 8.95 \\
\hline \multicolumn{6}{|l|}{ TNV05 } \\
\hline C1 & 1.00 & 0.00 & n.a. & n.a. & n.a. \\
\hline $\mathrm{C} 2$ & 1.00 & 0.00 & n.a. & n.a. & n.a. \\
\hline R1 & 1.05 & 4.55 & 2.00 & 14.29 & 30.30 \\
\hline $\mathrm{R} 2$ & 1.04 & 4.44 & 2.00 & 5.56 & 34.43 \\
\hline $\mathrm{RC} 1$ & 1.02 & 1.68 & 2.00 & 11.11 & 17.17 \\
\hline $\mathrm{RC} 2$ & 1.01 & 0.73 & 2.00 & 0.00 & 6.70 \\
\hline Avg. & 1.02 & 1.65 & 2.00 & 10.98 & 12.86 \\
\hline \multicolumn{6}{|l|}{ TNV75 } \\
\hline $\mathrm{C} 1$ & 1.00 & 0.00 & $n \cdot a$. & $n . a$. & $n . a$. \\
\hline $\mathrm{C} 2$ & 1.00 & 0.00 & n.a. & n.a. & $n . a$. \\
\hline $\mathrm{R} 1$ & 1.06 & 6.00 & 2.00 & 14.29 & 24.25 \\
\hline $\mathrm{R} 2$ & 1.06 & 6.22 & 2.00 & 5.56 & 28.66 \\
\hline $\mathrm{RC} 1$ & 1.03 & 2.72 & 2.00 & 4.17 & 24.36 \\
\hline $\mathrm{RC} 2$ & 1.02 & 1.45 & 2.00 & 16.67 & 7.08 \\
\hline Avg. & 1.02 & 2.37 & 2.00 & 10.00 & 12.85 \\
\hline
\end{tabular}


Table EC.12 Objective III - Effect of the different distribution policies on solution structure compared to VRPTW, grouped by Solomon class

\begin{tabular}{|c|c|c|c|c|c|}
\hline \multirow[b]{2}{*}{$\begin{array}{r}\text { Policy / } \\
\text { Solomon } \\
\text { class }\end{array}$} & \multicolumn{5}{|c|}{ Average of } \\
\hline & $\begin{array}{l}\text { Number of } \\
\text { visits per } \\
\text { customer }\end{array}$ & $\begin{array}{l}\text { Percentage of } \\
\text { split customers }\end{array}$ & $\begin{array}{l}\text { Number of } \\
\text { visits per split } \\
\text { customer }\end{array}$ & $\begin{array}{c}\text { Percentage of } \\
\text { split customers } \\
\text { with deliveries } \\
\text { fully } \\
\text { synchronized }\end{array}$ & $\begin{array}{c}\text { Timespan } \\
\text { between first and } \\
\text { last delivery in } \\
\text { relation to time } \\
\text { window width in } \\
\%\end{array}$ \\
\hline \multicolumn{6}{|l|}{ SDVRPTW } \\
\hline $\mathrm{C} 1$ & 1.01 & 0.74 & 2.00 & 20.00 & 5.37 \\
\hline $\mathrm{C} 2$ & 1.03 & 2.48 & 2.00 & 55.00 & 10.78 \\
\hline $\mathrm{R} 1$ & 1.08 & 8.36 & 2.00 & 8.73 & 33.66 \\
\hline $\mathrm{R} 2$ & 1.08 & 8.44 & 2.00 & 3.70 & 26.09 \\
\hline $\mathrm{RC} 1$ & 1.03 & 2.88 & 2.04 & 19.44 & 18.45 \\
\hline $\mathrm{RC} 2$ & 1.02 & 2.18 & 2.00 & 16.67 & 15.30 \\
\hline Avg. & 1.04 & 3.72 & 2.01 & 19.17 & 17.18 \\
\hline \multicolumn{6}{|l|}{ NV2 } \\
\hline $\mathrm{C} 1$ & 1.01 & 0.74 & 2.00 & 20.00 & 5.37 \\
\hline $\mathrm{C} 2$ & 1.03 & 2.48 & 2.00 & 30.00 & 17.22 \\
\hline $\mathrm{R} 1$ & 1.08 & 8.36 & 2.00 & 4.76 & 34.00 \\
\hline $\mathrm{R} 2$ & 1.08 & 8.44 & 2.00 & 0.00 & 30.60 \\
\hline $\mathrm{RC} 1$ & 1.03 & 2.88 & 2.00 & 11.11 & 22.40 \\
\hline $\mathrm{RC} 2$ & 1.02 & 2.18 & 2.00 & 16.67 & 11.29 \\
\hline Avg. & 1.04 & 3.72 & 2.00 & 11.39 & 19.25 \\
\hline \multicolumn{6}{|l|}{ So } \\
\hline $\mathrm{C} 1$ & 1.01 & 0.74 & 2.00 & 100.00 & 0.00 \\
\hline $\mathrm{C} 2$ & 1.03 & 2.48 & 2.00 & 100.00 & 0.00 \\
\hline $\mathrm{R} 1$ & 1.07 & 7.09 & 2.00 & 100.00 & 0.00 \\
\hline $\mathrm{R} 2$ & 1.08 & 7.56 & 2.00 & 100.00 & 0.00 \\
\hline $\mathrm{RC} 1$ & 1.02 & 1.92 & 2.07 & 100.00 & 0.00 \\
\hline $\mathrm{RC} 2$ & 1.01 & 1.09 & 2.00 & 100.00 & 0.00 \\
\hline Avg. & 1.03 & 3.10 & 2.01 & 100.00 & 0.00 \\
\hline \multicolumn{6}{|l|}{ TNV25 } \\
\hline $\mathrm{C} 1$ & 1.00 & 0.00 & n.a. & n.a. & n.a. \\
\hline $\mathrm{C} 2$ & 1.00 & 0.38 & 2.00 & 50.00 & 4.76 \\
\hline $\mathrm{R} 1$ & 1.03 & 2.91 & 2.00 & 12.50 & 24.52 \\
\hline $\mathrm{R} 2$ & 1.03 & 3.11 & 2.00 & 0.00 & 14.97 \\
\hline $\mathrm{RC} 1$ & 1.01 & 0.88 & 2.00 & 33.33 & 5.99 \\
\hline $\mathrm{RC} 2$ & 1.01 & 0.73 & 2.00 & 0.00 & 9.29 \\
\hline Avg. & 1.01 & 1.13 & 2.00 & 15.15 & 8.92 \\
\hline \multicolumn{6}{|l|}{ TNV50 } \\
\hline $\mathrm{C} 1$ & 1.01 & 0.74 & 2.00 & 20.00 & 5.37 \\
\hline $\mathrm{C} 2$ & 1.02 & 2.10 & 2.00 & 55.00 & 8.83 \\
\hline $\mathrm{R} 1$ & 1.05 & 5.09 & 2.00 & 9.52 & 35.16 \\
\hline $\mathrm{R} 2$ & 1.05 & 5.33 & 2.00 & 0.00 & 30.52 \\
\hline $\mathrm{RC} 1$ & 1.02 & 2.16 & 2.00 & 20.83 & 18.57 \\
\hline $\mathrm{RC} 2$ & 1.02 & 1.45 & 2.00 & 0.00 & 17.61 \\
\hline Avg. & 1.03 & 2.56 & 2.00 & 18.33 & 17.71 \\
\hline \multicolumn{6}{|l|}{ TNV75 } \\
\hline $\mathrm{C} 1$ & 1.01 & 0.74 & 2.00 & 20.00 & 5.37 \\
\hline $\mathrm{C} 2$ & 1.02 & 2.29 & 2.00 & 55.00 & 8.83 \\
\hline $\mathrm{R} 1$ & 1.07 & 6.73 & 2.00 & 4.76 & 31.85 \\
\hline $\mathrm{R} 2$ & 1.07 & 7.11 & 2.00 & 0.00 & 30.24 \\
\hline $\mathrm{RC} 1$ & 1.03 & 2.72 & 2.00 & 8.33 & 22.10 \\
\hline $\mathrm{RC} 2$ & 1.02 & 1.82 & 2.00 & 16.67 & 12.38 \\
\hline Avg. & 1.03 & 3.20 & 2.00 & 15.00 & 17.32 \\
\hline
\end{tabular}


Table EC.13 Objective I - Relative savings obtained with the different distribution policies compared to VRPTW, grouped by Solomon class

\begin{tabular}{|c|c|c|c|c|c|}
\hline \multirow[b]{2}{*}{$\begin{array}{r}\text { Policy / } \\
\text { Solomon } \\
\text { class }\end{array}$} & \multicolumn{5}{|c|}{ Min./Avg./Max. \% Savings/\# Instances with savings $>\mathbf{3 \%}$ in } \\
\hline & Objective value & Number of routes & $\begin{array}{c}\text { Variable routing } \\
\text { costs }\end{array}$ & Route durations & $\begin{array}{l}\text { Sum of variable } \\
\text { routing costs and } \\
\text { costs related to } \\
\text { route durations }\end{array}$ \\
\hline \multicolumn{6}{|l|}{ SDVRPTW } \\
\hline $\mathrm{C} 1$ & $1.03 / 3.03 / 8.87 / 9$ & $0.00 / 3.70 / 13.33 / 9$ & $1.03 / 3.03 / 8.87 / 9$ & $-58.50 /-19.69 / 4.27 / 1$ & $-49.71 /-16.70 / 3.92 / 1$ \\
\hline $\mathrm{C} 2$ & $0.76 / 3.90 / 8.51 / 14$ & $0.00 / 2.65 / 10.00 / 7$ & $0.76 / 3.90 / 8.51 / 14$ & $-81.47 /-9.29 / 16.99 / 4$ & $-70.56 /-7.45 / 15.84 / 4$ \\
\hline $\mathrm{R} 1$ & $0.00 / 3.08 / 4.61 / 13$ & $0.00 / 1.21 / 9.09 / 3$ & $0.00 / 3.08 / 4.61 / 13$ & $-6.99 /-1.38 / 2.61 / 0$ & $-2.65 / 0.48 / 2.82 / 0$ \\
\hline $\mathrm{R} 2$ & $1.40 / 3.10 / 4.00 / 5$ & $0.00 / 0.00 / 0.00 / 0$ & $1.40 / 3.10 / 4.00 / 5$ & $-23.21 /-6.88 / 8.99 / 2$ & $-17.73 /-3.70 / 7.12 / 2$ \\
\hline $\mathrm{RC} 1$ & $0.00 / 1.06 / 3.56 / 5$ & $0.00 / 2.23 / 7.14 / 8$ & $0.00 / 1.06 / 3.56 / 5$ & $-6.05 /-1.30 / 0.73 / 0$ & $-3.10 /-0.30 / 1.99 / 0$ \\
\hline $\mathrm{RC} 2$ & $0.00 / 0.65 / 3.56 / 1$ & $0.00 / 1.86 / 7.14 / 3$ & $0.00 / 0.65 / 3.56 / 1$ & $-47.37 /-5.31 / 10.07 / 1$ & $-28.79 /-3.40 / 6.50 / 1$ \\
\hline$A v g$ & $0.00 / 2.55 / 8.87 / 47$ & $0.00 / 2.25 / 13.33 / 30$ & $0.00 / 2.55 / 8.87 / 47$ & $-81.47 /-7.91 / 16.99 / 8$ & $-70.56 /-5.87 / 15.84 / 8$ \\
\hline \multicolumn{6}{|l|}{ NV2 } \\
\hline $\mathrm{C} 1$ & $1.03 / 3.03 / 8.87 / 9$ & $0.00 / 3.70 / 13.33 / 9$ & $1.03 / 3.03 / 8.87 / 9$ & $-58.50 /-20.04 / 4.27 / 1$ & $-49.71 /-17.01 / 3.92 / 1$ \\
\hline $\mathrm{C} 2$ & $0.76 / 3.90 / 8.51 / 14$ & $0.00 / 2.65 / 10.00 / 7$ & $0.76 / 3.90 / 8.51 / 14$ & $-81.47 /-8.89 / 18.46 / 4$ & $-70.56 /-7.08 / 17.19 / 4$ \\
\hline $\mathrm{R} 1$ & $0.00 / 3.08 / 4.61 / 13$ & $0.00 / 1.21 / 9.09 / 3$ & $0.00 / 3.08 / 4.61 / 13$ & $-6.99 /-1.18 / 3.96 / 1$ & $-2.65 / 0.60 / 3.55 / 1$ \\
\hline $\mathrm{R} 2$ & $1.40 / 3.10 / 4.00 / 5$ & $0.00 / 0.00 / 0.00 / 0$ & $1.40 / 3.10 / 4.00 / 5$ & $-23.99 /-7.19 / 8.99 / 2$ & $-17.73 /-3.90 / 7.12 / 2$ \\
\hline $\mathrm{RC} 1$ & $0.00 / 1.06 / 3.56 / 5$ & $0.00 / 2.23 / 7.14 / 8$ & $0.00 / 1.06 / 3.56 / 5$ & $-6.05 /-1.29 / 0.73 / 0$ & $-3.10 /-0.29 / 1.99 / 0$ \\
\hline $\mathrm{RC} 2$ & $0.00 / 0.65 / 3.56 / 1$ & $0.00 / 1.86 / 7.14 / 3$ & $0.00 / 0.65 / 3.56 / 1$ & $-47.37 /-5.42 / 10.07 / 1$ & $-28.79 /-3.47 / 6.50 / 1$ \\
\hline Avg. & $0.00 / 2.55 / 8.87 / 47$ & $0.00 / 2.25 / 13.33 / 30$ & $0.00 / 2.55 / 8.87 / 47$ & $-81.47 /-7.92 / 18.46 / 9$ & $-70.56 /-5.87 / 17.19 / 9$ \\
\hline \multicolumn{6}{|l|}{ So } \\
\hline $\mathrm{C} 1$ & $1.03 / 3.03 / 8.87 / 9$ & $0.00 / 3.70 / 13.33 / 9$ & $1.03 / 3.03 / 8.87 / 9$ & $-68.38 /-40.74 /-11.80 / 0$ & $-58.96 /-35.11 /-10.69 / 0$ \\
\hline $\mathrm{C} 2$ & $0.76 / 3.90 / 8.51 / 14$ & $0.00 / 2.65 / 10.00 / 7$ & $0.76 / 3.90 / 8.51 / 14$ & $-402.52 /-107.18 / 2.31 / 0$ & $-332.27 /-91.43 / 2.39 / 0$ \\
\hline $\mathrm{R} 1$ & $0.00 / 2.87 / 4.00 / 9$ & $0.00 / 1.21 / 9.09 / 3$ & $0.00 / 2.87 / 4.00 / 9$ & $-43.38 /-24.70 / 0.00 / 0$ & $-24.90 /-13.36 / 0.00 / 0$ \\
\hline $\mathrm{R} 2$ & $1.40 / 3.06 / 4.00 / 4$ & $0.00 / 0.00 / 0.00 / 0$ & $1.40 / 3.06 / 4.00 / 4$ & $-240.19 /-142.72 /-58.02 / 0$ & $-136.52 /-90.71 /-45.93 / 0$ \\
\hline $\mathrm{RC} 1$ & $0.00 / 1.06 / 3.56 / 5$ & $0.00 / 2.23 / 7.14 / 8$ & $0.00 / 1.06 / 3.56 / 5$ & $-30.26 /-13.04 / 0.00 / 0$ & $-18.30 /-7.09 / 0.00 / 0$ \\
\hline $\mathrm{RC} 2$ & $0.00 / 0.65 / 3.56 / 1$ & $0.00 / 1.86 / 7.14 / 3$ & $0.00 / 0.65 / 3.56 / 1$ & $-119.68 /-67.85 / 0.00 / 0$ & $-74.45 /-43.75 / 0.00 / 0$ \\
\hline$A v g$ & $0.00 / 2.50 / 8.87 / 42$ & $0.00 / 2.25 / 13.33 / 30$ & $0.00 / 2.50 / 8.87 / 42$ & $-402.52 /-54.36 / 2.31 / 0$ & $-332.27 /-40.32 / 2.39 / 0$ \\
\hline \multicolumn{6}{|l|}{ TNV25 } \\
\hline $\mathrm{C} 1$ & $0.00 / 1.58 / 3.57 / 6$ & $0.00 / 2.96 / 10.00 / 9$ & $0.00 / 1.58 / 3.57 / 6$ & $-41.94 /-13.72 / 3.41 / 1$ & $-35.72 /-11.65 / 3.13 / 1$ \\
\hline $\mathrm{C} 2$ & $0.00 / 2.40 / 5.49 / 10$ & $0.00 / 0.95 / 10.00 / 2$ & $0.00 / 2.40 / 5.49 / 10$ & $-46.82 / 0.24 / 27.69 / 7$ & $-38.40 / 0.55 / 25.14 / 7$ \\
\hline $\mathrm{R} 1$ & $0.00 / 1.49 / 2.67 / 0$ & $-10.00 /-3.18 / 0.00 / 0$ & $0.00 / 1.49 / 2.67 / 0$ & $-6.05 / 0.32 / 3.89 / 2$ & $-2.45 / 0.80 / 2.95 / 0$ \\
\hline $\mathrm{R} 2$ & $1.26 / 1.71 / 2.00 / 0$ & $-10.00 /-3.33 / 0.00 / 0$ & $1.26 / 1.71 / 2.00 / 0$ & $-34.49 / 1.58 / 24.48 / 3$ & $-27.22 / 1.03 / 16.21 / 3$ \\
\hline $\mathrm{RC} 1$ & $0.00 / 0.24 / 1.33 / 0$ & $0.00 / 0.00 / 0.00 / 0$ & $0.00 / 0.24 / 1.33 / 0$ & $-3.06 /-0.76 / 0.60 / 0$ & $-1.65 /-0.34 / 0.92 / 0$ \\
\hline $\mathrm{RC} 2$ & $0.00 / 0.18 / 0.44 / 0$ & $0.00 / 0.00 / 0.00 / 0$ & $0.00 / 0.18 / 0.44 / 0$ & $-16.42 /-2.75 / 0.09 / 0$ & $-10.34 /-1.80 / 0.08 / 0$ \\
\hline$A v g$ & $0.00 / 1.30 / 5.49 / 16$ & $-10.00 / 0.00 / 10.00 / 11$ & $0.00 / 1.30 / 5.49 / 16$ & $-46.82 /-3.42 / 27.69 / 13$ & $-38.40 /-2.65 / 25.14 / 11$ \\
\hline \multicolumn{6}{|l|}{ TNV50 } \\
\hline $\mathrm{C} 1$ & $0.92 / 2.78 / 8.19 / 9$ & $0.00 / 3.70 / 13.33 / 9$ & $0.92 / 2.78 / 8.19 / 9$ & $-42.10 /-15.16 / 3.41 / 1$ & $-35.64 /-12.82 / 3.13 / 1$ \\
\hline $\mathrm{C} 2$ & $0.66 / 3.60 / 7.85 / 13$ & $0.00 / 2.65 / 10.00 / 7$ & $0.66 / 3.60 / 7.85 / 13$ & $-31.76 /-5.35 / 18.46 / 4$ & $-27.45 /-4.11 / 17.19 / 4$ \\
\hline $\mathrm{R} 1$ & $0.00 / 2.27 / 2.89 / 0$ & $0.00 / 0.79 / 9.09 / 2$ & $0.00 / 2.27 / 2.89 / 0$ & $-6.05 / 0.39 / 6.56 / 5$ & $-2.45 / 1.17 / 4.96 / 3$ \\
\hline $\mathrm{R} 2$ & $1.26 / 2.24 / 2.70 / 0$ & $0.00 / 0.00 / 0.00 / 0$ & $1.26 / 2.24 / 2.70 / 0$ & $-34.49 / 2.26 / 34.26 / 2$ & $-27.22 / 2.00 / 24.15 / 2$ \\
\hline $\mathrm{RC} 1$ & $0.00 / 0.85 / 3.00 / 2$ & $0.00 / 1.96 / 7.14 / 7$ & $0.00 / 0.85 / 3.00 / 2$ & $-3.06 /-0.66 / 0.95 / 0$ & $-1.65 /-0.02 / 1.86 / 0$ \\
\hline $\mathrm{RC} 2$ & $0.00 / 0.50 / 3.03 / 1$ & $0.00 / 1.86 / 7.14 / 3$ & $0.00 / 0.50 / 3.03 / 1$ & $-28.65 /-4.01 / 0.00 / 0$ & $-17.10 /-2.48 / 0.00 / 0$ \\
\hline Avg. & $0.00 / 2.15 / 8.19 / 25$ & $0.00 / 2.11 / 13.33 / 28$ & $0.00 / 2.15 / 8.19 / 25$ & $-42.10 /-4.81 / 34.26 / 12$ & $-35.64 /-3.62 / 24.15 / 10$ \\
\hline \multicolumn{6}{|l|}{ TNV75 } \\
\hline $\mathrm{C} 1$ & $1.03 / 2.91 / 8.45 / 9$ & $0.00 / 3.70 / 13.33 / 9$ & $1.03 / 2.91 / 8.45 / 9$ & $-56.30 /-18.89 / 4.27 / 1$ & $-47.81 /-16.04 / 3.92 / 1$ \\
\hline $\mathrm{C} 2$ & $0.66 / 3.73 / 8.17 / 13$ & $0.00 / 2.65 / 10.00 / 7$ & $0.66 / 3.73 / 8.17 / 13$ & $-31.76 /-6.58 / 18.46 / 4$ & $-27.45 /-5.16 / 17.19 / 4$ \\
\hline $\mathrm{R} 1$ & $0.00 / 2.88 / 4.41 / 9$ & $0.00 / 1.21 / 9.09 / 3$ & $0.00 / 2.88 / 4.41 / 9$ & $-6.99 / 0.26 / 6.56 / 4$ & $-2.65 / 1.35 / 4.96 / 3$ \\
\hline $\mathrm{R} 2$ & $1.40 / 2.90 / 3.80 / 3$ & $0.00 / 0.00 / 0.00 / 0$ & $1.40 / 2.90 / 3.80 / 3$ & $-23.21 /-1.02 / 34.26 / 2$ & $-17.73 / 0.26 / 24.15 / 2$ \\
\hline $\mathrm{RC} 1$ & $0.00 / 1.02 / 3.41 / 5$ & $0.00 / 1.96 / 7.14 / 7$ & $0.00 / 1.02 / 3.41 / 5$ & $-5.49 /-1.21 / 0.55 / 0$ & $-3.10 /-0.26 / 1.82 / 0$ \\
\hline $\mathrm{RC} 2$ & $0.00 / 0.63 / 3.41 / 1$ & $0.00 / 1.86 / 7.14 / 3$ & $0.00 / 0.63 / 3.41 / 1$ & $-47.55 /-5.49 / 8.56 / 1$ & $-28.96 /-3.52 / 5.57 / 1$ \\
\hline Avg. & $0.00 / 2.42 / 8.45 / 40$ & $0.00 / 2.19 / 13.33 / 29$ & $0.00 / 2.42 / 8.45 / 40$ & $-56.30 /-6.46 / 34.26 / 12$ & $-47.81 /-4.82 / 24.15 / 11$ \\
\hline
\end{tabular}


Table EC.14 Objective II - Relative savings obtained with the different distribution policies compared to VRPTW, grouped by Solomon class

\begin{tabular}{|c|c|c|c|c|c|}
\hline \multirow[b]{2}{*}{$\begin{array}{r}\text { Policy / } \\
\text { Solomon } \\
\text { class }\end{array}$} & \multicolumn{5}{|c|}{ Min./Avg./Max. \% Savings/\# Instances with savings $>3 \%$ in } \\
\hline & Objective value & Number of routes & $\begin{array}{l}\text { Variable routing } \\
\text { costs }\end{array}$ & Route durations & $\begin{array}{l}\text { Sum of variable } \\
\text { routing costs and } \\
\text { costs related to } \\
\text { route durations }\end{array}$ \\
\hline \multicolumn{6}{|l|}{ SDVRPTW } \\
\hline $\mathrm{C} 1$ & $0.00 / 0.00 / 0.00 / 0$ & $0.00 / 0.00 / 0.00 / 0$ & $0.00 / 0.00 / 0.00 / 0$ & $0.00 / 0.00 / 0.00 / 0$ & $0.00 / 0.00 / 0.00 / 0$ \\
\hline $\mathrm{C} 2$ & $0.00 / 0.00 / 0.00 / 0$ & $0.00 / 0.00 / 0.00 / 0$ & $0.00 / 0.00 / 0.00 / 0$ & $0.00 / 0.00 / 0.00 / 0$ & $0.00 / 0.00 / 0.00 / 0$ \\
\hline $\mathrm{R} 1$ & $0.00 / 1.24 / 1.94 / 0$ & $0.00 / 2.53 / 10.00 / 6$ & $0.00 / 2.93 / 4.34 / 12$ & $-1.66 / 0.01 / 0.95 / 0$ & $0.00 / 1.24 / 1.94 / 0$ \\
\hline $\mathrm{R} 2$ & $0.67 / 1.32 / 1.93 / 0$ & $0.00 / 4.04 / 18.18 / 3$ & $1.59 / 3.41 / 4.86 / 6$ & $-2.34 /-0.20 / 0.29 / 0$ & $0.67 / 1.32 / 1.93 / 0$ \\
\hline $\mathrm{RC} 1$ & $0.00 / 0.46 / 2.07 / 0$ & $0.00 / 1.43 / 7.14 / 5$ & $-1.15 / 0.75 / 3.60 / 3$ & $-0.80 / 0.23 / 1.27 / 0$ & $0.00 / 0.46 / 2.07 / 0$ \\
\hline $\mathrm{RC} 2$ & $0.00 / 0.28 / 1.53 / 0$ & $0.00 / 0.65 / 7.14 / 1$ & $0.00 / 0.38 / 2.60 / 0$ & $-0.43 / 0.20 / 2.01 / 0$ & $0.00 / 0.28 / 1.53 / 0$ \\
\hline$A v g$ & $0.00 / 0.47 / 2.07 / 0$ & $0.00 / 1.17 / 18.18 / 15$ & $-1.15 / 1.03 / 4.86 / 21$ & $-2.34 / 0.06 / 2.01 / 0$ & $0.00 / 0.47 / 2.07 / 0$ \\
\hline \multicolumn{6}{|l|}{ NV2 } \\
\hline $\mathrm{C} 1$ & $0.00 / 0.00 / 0.00 / 0$ & $0.00 / 0.00 / 0.00 / 0$ & $0.00 / 0.00 / 0.00 / 0$ & $0.00 / 0.00 / 0.00 / 0$ & $0.00 / 0.00 / 0.00 / 0$ \\
\hline $\mathrm{C} 2$ & $0.00 / 0.00 / 0.00 / 0$ & $0.00 / 0.00 / 0.00 / 0$ & $0.00 / 0.00 / 0.00 / 0$ & $0.00 / 0.00 / 0.00 / 0$ & $0.00 / 0.00 / 0.00 / 0$ \\
\hline $\mathrm{R} 1$ & $0.00 / 1.24 / 1.94 / 0$ & $0.00 / 2.53 / 10.00 / 6$ & $0.00 / 2.93 / 4.34 / 12$ & $-1.66 / 0.01 / 0.95 / 0$ & $0.00 / 1.24 / 1.94 / 0$ \\
\hline $\mathrm{R} 2$ & $0.67 / 1.32 / 1.93 / 0$ & $0.00 / 4.04 / 18.18 / 3$ & $1.59 / 3.41 / 4.86 / 6$ & $-2.34 /-0.20 / 0.29 / 0$ & $0.67 / 1.32 / 1.93 / 0$ \\
\hline $\mathrm{RC} 1$ & $0.00 / 0.46 / 2.07 / 0$ & $0.00 / 1.43 / 7.14 / 5$ & $-1.15 / 0.73 / 3.18 / 3$ & $-0.80 / 0.24 / 1.27 / 0$ & $0.00 / 0.46 / 2.07 / 0$ \\
\hline $\mathrm{RC} 2$ & $0.00 / 0.28 / 1.53 / 0$ & $0.00 / 0.65 / 7.14 / 1$ & $0.00 / 0.38 / 2.60 / 0$ & $-0.43 / 0.20 / 2.01 / 0$ & $0.00 / 0.28 / 1.53 / 0$ \\
\hline$A v g$ & $0.00 / 0.47 / 2.07 / 0$ & $0.00 / 1.17 / 18.18 / 15$ & $-1.15 / 1.02 / 4.86 / 21$ & $-2.34 / 0.06 / 2.01 / 0$ & $0.00 / 0.47 / 2.07 / 0$ \\
\hline \multicolumn{6}{|c|}{ 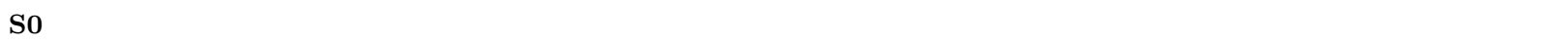 } \\
\hline $\mathrm{C} 1$ & $0.00 / 0.00 / 0.00 / 0$ & $0.00 / 0.00 / 0.00 / 0$ & $0.00 / 0.00 / 0.00 / 0$ & $0.00 / 0.00 / 0.00 / 0$ & $0.00 / 0.00 / 0.00 / 0$ \\
\hline $\mathrm{C} 2$ & $0.00 / 0.00 / 0.00 / 0$ & $0.00 / 0.00 / 0.00 / 0$ & $0.00 / 0.00 / 0.00 / 0$ & $0.00 / 0.00 / 0.00 / 0$ & $0.00 / 0.00 / 0.00 / 0$ \\
\hline $\mathrm{R} 1$ & $0.00 / 1.05 / 1.93 / 0$ & $-10.00 / 1.62 / 10.00 / 6$ & $0.00 / 2.49 / 4.34 / 8$ & $-1.66 / 0.01 / 0.95 / 0$ & $0.00 / 1.05 / 1.93 / 0$ \\
\hline $\mathrm{R} 2$ & $0.00 / 1.09 / 1.93 / 0$ & $0.00 / 4.04 / 18.18 / 3$ & $0.00 / 3.10 / 4.86 / 6$ & $-2.85 /-0.38 / 0.29 / 0$ & $0.00 / 1.09 / 1.93 / 0$ \\
\hline Avg. & $0.00 / 0.40 / 2.06 / 0$ & $-10.00 / 1.00 / 18.18 / 15$ & $0.00 / 0.91 / 4.86 / 17$ & $-2.85 / 0.03 / 1.72 / 0$ & $0.00 / 0.40 / 2.06 / 0$ \\
\hline \multicolumn{6}{|l|}{ TNV25 } \\
\hline $\mathrm{C} 1$ & $0.00 / 0.00 / 0.00 / 0$ & $0.00 / 0.00 / 0.00 / 0$ & $0.00 / 0.00 / 0.00 / 0$ & $0.00 / 0.00 / 0.00 / 0$ & $0.00 / 0.00 / 0.00 / 0$ \\
\hline $\mathrm{C} 2$ & $0.00 / 0.00 / 0.00 / 0$ & $0.00 / 0.00 / 0.00 / 0$ & $0.00 / 0.00 / 0.00 / 0$ & $0.00 / 0.00 / 0.00 / 0$ & $0.00 / 0.00 / 0.00 / 0$ \\
\hline $\mathrm{R} 1$ & $0.00 / 0.72 / 1.71 / 0$ & $-10.00 /-1.40 / 10.00 / 3$ & $0.00 / 1.26 / 2.82 / 0$ & $-0.97 / 0.33 / 1.31 / 0$ & $0.00 / 0.72 / 1.71 / 0$ \\
\hline $\mathrm{R} 2$ & $0.00 / 0.76 / 1.36 / 0$ & $-10.00 /-0.10 / 9.09 / 1$ & $0.00 / 1.47 / 2.41 / 0$ & $-0.08 / 0.25 / 0.60 / 0$ & $0.00 / 0.76 / 1.36 / 0$ \\
\hline $\mathrm{RC} 1$ & $0.00 / 0.05 / 0.92 / 0$ & $0.00 / 0.00 / 0.00 / 0$ & $0.00 / 0.09 / 1.33 / 0$ & $-0.21 / 0.02 / 0.60 / 0$ & $0.00 / 0.05 / 0.92 / 0$ \\
\hline $\mathrm{RC} 2$ & $0.00 / 0.00 / 0.00 / 0$ & $0.00 / 0.00 / 0.00 / 0$ & $0.00 / 0.00 / 0.00 / 0$ & $0.00 / 0.00 / 0.00 / 0$ & $0.00 / 0.00 / 0.00 / 0$ \\
\hline$A v g$ & $0.00 / 0.21 / 1.71 / 0$ & $-10.00 /-0.28 / 10.00 / 4$ & $0.00 / 0.37 / 2.82 / 0$ & $-0.97 / 0.09 / 1.31 / 0$ & $0.00 / 0.21 / 1.71 / 0$ \\
\hline \multicolumn{6}{|l|}{ TNV50 } \\
\hline $\mathrm{C} 1$ & $0.00 / 0.00 / 0.00 / 0$ & $0.00 / 0.00 / 0.00 / 0$ & $0.00 / 0.00 / 0.00 / 0$ & $0.00 / 0.00 / 0.00 / 0$ & $0.00 / 0.00 / 0.00 / 0$ \\
\hline $\mathrm{C} 2$ & $0.00 / 0.00 / 0.00 / 0$ & $0.00 / 0.00 / 0.00 / 0$ & $0.00 / 0.00 / 0.00 / 0$ & $0.00 / 0.00 / 0.00 / 0$ & $0.00 / 0.00 / 0.00 / 0$ \\
\hline $\mathrm{R} 1$ & $0.00 / 0.91 / 1.71 / 0$ & $-10.00 / 1.21 / 10.00 / 5$ & $0.00 / 1.88 / 2.82 / 0$ & $-1.66 / 0.21 / 1.31 / 0$ & $0.00 / 0.91 / 1.71 / 0$ \\
\hline $\mathrm{R} 2$ & $0.46 / 0.99 / 1.36 / 0$ & $0.00 / 1.01 / 9.09 / 1$ & $1.19 / 1.99 / 2.56 / 0$ & $-0.18 / 0.26 / 0.60 / 0$ & $0.46 / 0.99 / 1.36 / 0$ \\
\hline $\mathrm{RC} 1$ & $0.00 / 0.23 / 2.01 / 0$ & $0.00 / 0.57 / 7.14 / 2$ & $-1.15 / 0.34 / 3.14 / 1$ & $-0.80 / 0.15 / 1.45 / 0$ & $0.00 / 0.23 / 2.01 / 0$ \\
\hline $\mathrm{RC} 2$ & $0.00 / 0.14 / 1.42 / 0$ & $0.00 / 0.00 / 0.00 / 0$ & $0.00 / 0.15 / 0.94 / 0$ & $-0.43 / 0.14 / 2.01 / 0$ & $0.00 / 0.14 / 1.42 / 0$ \\
\hline$A v g$ & $0.00 / 0.32 / 2.01 / 0$ & $-10.00 / 0.43 / 10.00 / 8$ & $-1.15 / 0.60 / 3.14 / 1$ & $-1.66 / 0.11 / 2.01 / 0$ & $0.00 / 0.32 / 2.01 / 0$ \\
\hline \multicolumn{6}{|l|}{ TNV75 } \\
\hline $\mathrm{C} 1$ & $0.00 / 0.00 / 0.00 / 0$ & $0.00 / 0.00 / 0.00 / 0$ & $0.00 / 0.00 / 0.00 / 0$ & $0.00 / 0.00 / 0.00 / 0$ & $0.00 / 0.00 / 0.00 / 0$ \\
\hline $\mathrm{C} 2$ & $0.00 / 0.00 / 0.00 / 0$ & $0.00 / 0.00 / 0.00 / 0$ & $0.00 / 0.00 / 0.00 / 0$ & $0.00 / 0.00 / 0.00 / 0$ & $0.00 / 0.00 / 0.00 / 0$ \\
\hline $\mathrm{R} 1$ & $0.00 / 1.04 / 1.94 / 0$ & $-10.00 / 1.21 / 10.00 / 5$ & $0.00 / 2.26 / 3.89 / 4$ & $-1.66 / 0.16 / 0.95 / 0$ & $0.00 / 1.04 / 1.94 / 0$ \\
\hline $\mathrm{R} 2$ & $0.67 / 1.11 / 1.59 / 0$ & $0.00 / 2.02 / 18.18 / 1$ & $1.59 / 2.77 / 4.86 / 3$ & $-2.34 /-0.10 / 0.52 / 0$ & $0.67 / 1.11 / 1.59 / 0$ \\
\hline $\mathrm{RC} 1$ & $0.00 / 0.46 / 2.01 / 0$ & $0.00 / 1.43 / 7.14 / 5$ & $-1.15 / 0.71 / 3.14 / 2$ & $-0.80 / 0.25 / 1.45 / 0$ & $0.00 / 0.46 / 2.01 / 0$ \\
\hline $\mathrm{RC} 2$ & $0.00 / 0.28 / 1.53 / 0$ & $0.00 / 0.65 / 7.14 / 1$ & $0.00 / 0.38 / 2.60 / 0$ & $-0.43 / 0.20 / 2.01 / 0$ & $0.00 / 0.28 / 1.53 / 0$ \\
\hline
\end{tabular}


Table EC.15 Objective III - Relative savings obtained with the different distribution policies compared to VRPTW, grouped by Solomon class

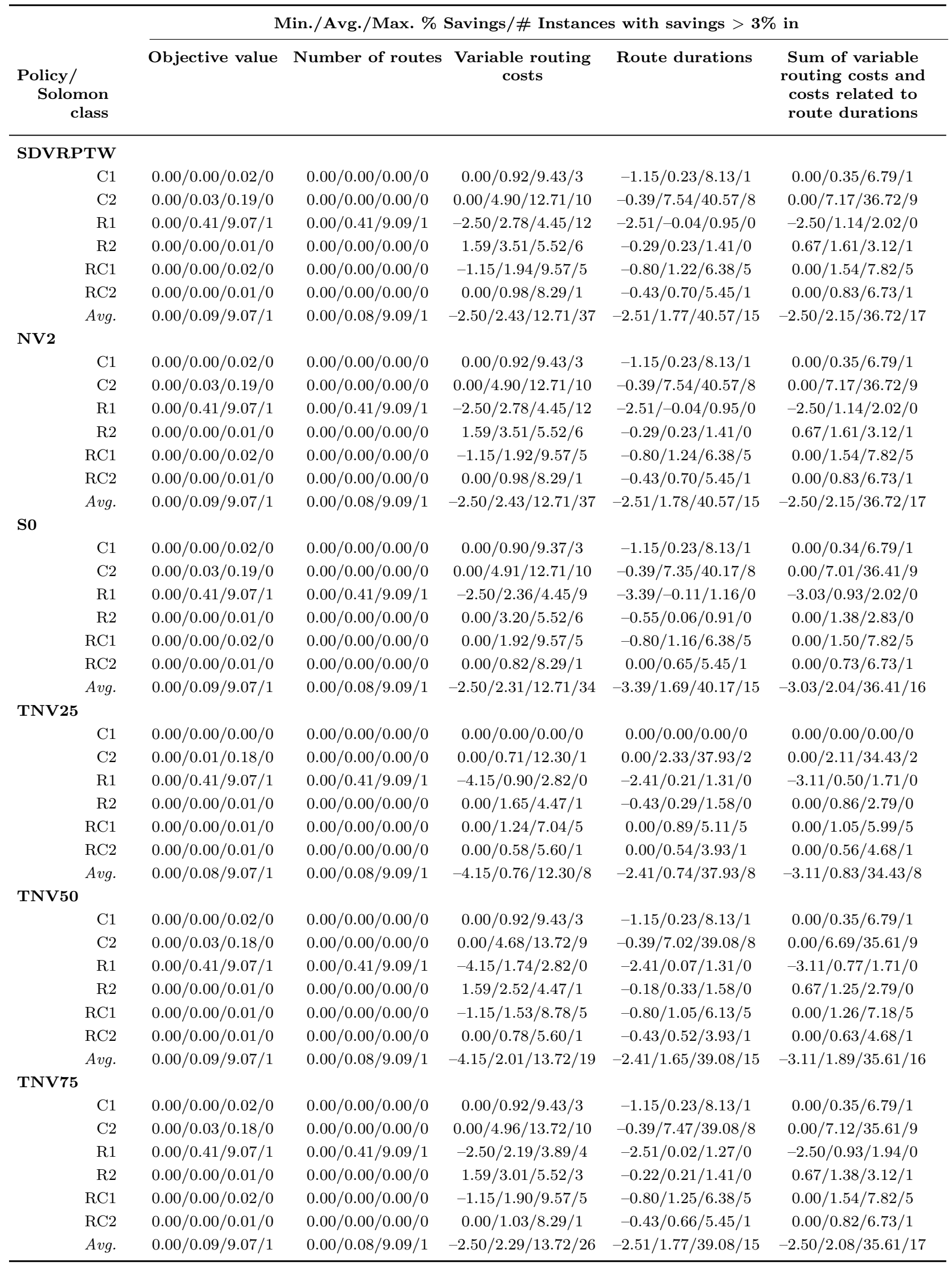


Table EC.16 Impact of inconvenience constraints on algorithm performance for objective I, grouped by Solomon class

\begin{tabular}{|c|c|c|c|c|c|c|c|c|c|c|c|}
\hline \multirow[b]{2}{*}{$\begin{array}{r}\text { Policy / } \\
\text { Solomon } \\
\text { class }\end{array}$} & \multirow[b]{2}{*}{$\begin{array}{l}\text { Num. } \\
\text { opt. } \\
\text { sols. }\end{array}$} & \multicolumn{10}{|c|}{ Average of } \\
\hline & & $\begin{array}{l}\text { Run } \\
\text { time }\end{array}$ & $\begin{array}{c}\text { B\&B } \\
\text { nodes }\end{array}$ & $\begin{array}{l}\text { Cap. } \\
\text { cuts }\end{array}$ & $\begin{array}{c}\text { 2-path } \\
\text { cuts }\end{array}$ & $\begin{array}{c}\text { Conn. } \\
\text { cuts }\end{array}$ & $\begin{array}{l}\text { Inf.- } \\
\text { path. } \\
\text { cuts }\end{array}$ & $\begin{array}{l}\text { Path- } \\
\text { match. } \\
\text { cuts }\end{array}$ & $\begin{array}{c}\text { Feas. } \\
\text { cuts }\end{array}$ & $\begin{array}{c}\text { Re } \\
\text { separa } \\
\text { streng } \\
\text { cuts }\end{array}$ & $\begin{array}{l}\text { tive } \\
\text { feas. } \\
\text { cuts }\end{array}$ \\
\hline \multicolumn{12}{|l|}{ SDVRPTW } \\
\hline $\mathrm{C} 1$ & 83 & 263 & 3,865 & 2,200 & 0.8 & 0.3 & 38.9 & 11.7 & 83.3 & 6.56 & 4.18 \\
\hline $\mathrm{C} 2$ & 39 & 116 & 3,420 & 1,115 & 0.0 & 1.0 & 50.3 & 23.6 & 188.0 & 6.52 & 13.37 \\
\hline $\mathrm{R} 1$ & 63 & 63 & 1,330 & 883 & 1.2 & 0.1 & 11.0 & 9.9 & 9.4 & 8.65 & 1.34 \\
\hline $\mathrm{R} 2$ & 57 & 94 & 1,863 & 884 & 0.0 & 0.1 & 5.8 & 1.9 & 7.5 & 7.22 & 1.60 \\
\hline $\mathrm{RC} 1$ & 77 & 99 & 3,164 & 1,480 & 0.7 & 0.9 & 27.0 & 8.3 & 98.9 & 9.79 & 2.41 \\
\hline $\mathrm{RC} 2$ & 77 & 112 & 2,257 & 1,495 & 0.0 & 0.6 & 17.6 & 3.8 & 101.2 & 6.88 & 3.94 \\
\hline Tot./Avg. & 396 & 131 & 2,681 & 1,417 & 0.5 & 0.5 & 24.4 & 9.0 & 77.5 & 7.67 & 3.87 \\
\hline \multicolumn{12}{|l|}{ NV2 } \\
\hline $\mathrm{C} 1$ & 83 & 214 & 3,191 & 2,098 & 0.7 & 0.5 & 30.6 & 7.7 & 24.8 & 6.72 & 3.24 \\
\hline $\mathrm{C} 2$ & 39 & 116 & 2,288 & 1,085 & 0.0 & 0.7 & 31.4 & 12.0 & 124.7 & 5.94 & 5.77 \\
\hline $\mathrm{R} 1$ & 63 & 81 & 1,235 & 870 & 1.1 & 0.1 & 8.6 & 8.1 & 2.5 & 8.59 & 0.77 \\
\hline $\mathrm{R} 2$ & 56 & 122 & 1,754 & 942 & 0.0 & 0.1 & 5.5 & 2.2 & 4.4 & 7.83 & 1.07 \\
\hline $\mathrm{RC} 1$ & 77 & 94 & 3,370 & 1,583 & 0.6 & 0.6 & 23.4 & 6.3 & 63.0 & 10.18 & 1.80 \\
\hline $\mathrm{RC} 2$ & 77 & 67 & 1,954 & 1,528 & 0.0 & 0.4 & 14.5 & 1.8 & 41.3 & 7.55 & 1.95 \\
\hline Tot./Avg. & 395 & 118 & 2,378 & 1,425 & 0.4 & 0.4 & 19.0 & 6.0 & 38.8 & 7.93 & 2.25 \\
\hline \multicolumn{12}{|l|}{ So } \\
\hline $\mathrm{C} 1$ & 81 & 394 & 4,650 & 2,184 & 0.9 & 0.4 & 41.1 & 10.3 & 123.3 & 6.09 & 8.30 \\
\hline $\mathrm{C} 2$ & 39 & 96 & 3,285 & 1,081 & 0.0 & 0.9 & 40.6 & 15.5 & 210.0 & 6.08 & 13.34 \\
\hline $\mathrm{R} 1$ & 61 & 127 & 2,168 & 1,012 & 1.9 & 0.1 & 16.0 & 17.4 & 27.4 & 9.02 & 3.13 \\
\hline $\mathrm{R} 2$ & 56 & 100 & 1,845 & 942 & 0.0 & 0.2 & 7.7 & 4.0 & 19.5 & 7.10 & 2.77 \\
\hline $\mathrm{RC} 1$ & 73 & 279 & 7,382 & 1,566 & 0.6 & 1.1 & 38.2 & 12.9 & 187.4 & 8.99 & 3.83 \\
\hline $\mathrm{RC} 2$ & 77 & 105 & 2,478 & 1,570 & 0.0 & 0.7 & 22.9 & 5.8 & 115.7 & 6.76 & 4.70 \\
\hline Tot./Avg. & 387 & 202 & 3,826 & 1,471 & 0.6 & 0.6 & 28.1 & 10.7 & 112.6 & 7.39 & 5.61 \\
\hline \multicolumn{12}{|l|}{ TNV25 } \\
\hline $\mathrm{C} 1$ & 39 & 2,089 & 8,259 & 10,685 & 2.5 & 0.1 & 44.7 & 0.3 & 0.0 & 4.40 & 0.05 \\
\hline $\mathrm{C} 2$ & 31 & 1,089 & 11,620 & 4,519 & 0.0 & 0.0 & 39.5 & 0.4 & 0.0 & 5.21 & 0.09 \\
\hline $\mathrm{R} 1$ & 47 & 1,213 & 14,526 & 6,355 & 2.4 & 0.0 & 27.6 & 1.5 & 0.0 & 5.74 & 0.05 \\
\hline $\mathrm{R} 2$ & 40 & 1,347 & 17,467 & 6,694 & 0.0 & 0.0 & 19.0 & 0.6 & 0.1 & 5.04 & 0.05 \\
\hline $\mathrm{RC} 1$ & 51 & 1,362 & 8,480 & 8,816 & 2.7 & 0.1 & 44.3 & 0.7 & 0.0 & 5.25 & 0.09 \\
\hline $\mathrm{RC} 2$ & 53 & 1,328 & 7,226 & 8,480 & 0.0 & 0.1 & 35.2 & 0.7 & 0.0 & 4.48 & 0.12 \\
\hline Tot./Avg. & 261 & 1,455 & 10,755 & 8,022 & 1.4 & 0.1 & 35.8 & 0.7 & 0.0 & 4.97 & 0.07 \\
\hline \multicolumn{12}{|l|}{ TNV50 } \\
\hline $\mathrm{C} 1$ & 50 & 1,593 & 12,173 & 7,658 & 1.9 & 0.2 & 59.1 & 2.1 & 0.0 & 5.17 & 0.19 \\
\hline $\mathrm{C} 2$ & 33 & 710 & 11,246 & 2,746 & 0.0 & 0.1 & 45.5 & 1.1 & 1.7 & 5.26 & 0.33 \\
\hline $\mathrm{R} 1$ & 49 & 979 & 16,263 & 4,356 & 2.2 & 0.0 & 31.9 & 6.2 & 0.0 & 7.39 & 0.12 \\
\hline $\mathrm{R} 2$ & 44 & 996 & 16,729 & 4,138 & 0.0 & 0.0 & 17.6 & 2.4 & 0.1 & 6.57 & 0.08 \\
\hline $\mathrm{RC} 1$ & 53 & 1,158 & 7,783 & 6,934 & 2.3 & 0.3 & 52.8 & 1.8 & 0.0 & 6.48 & 0.14 \\
\hline $\mathrm{RC} 2$ & 55 & 1,042 & 5,163 & 6,489 & 0.0 & 0.1 & 39.6 & 1.2 & 0.1 & 5.12 & 0.22 \\
\hline Tot./Avg. & 284 & 1,130 & 11,172 & 5,774 & 1.2 & 0.1 & 42.4 & 2.5 & 0.2 & 5.98 & 0.17 \\
\hline \multicolumn{12}{|l|}{ TNV75 } \\
\hline $\mathrm{C} 1$ & 66 & 948 & 5,386 & 5,091 & 1.2 & 0.2 & 48.7 & 3.0 & 0.5 & 6.30 & 0.29 \\
\hline $\mathrm{C} 2$ & 39 & 162 & 5,152 & 1,512 & 0.0 & 0.1 & 26.0 & 1.3 & 2.1 & 5.67 & 0.43 \\
\hline $\mathrm{R} 1$ & 59 & 475 & 26,252 & 1,645 & 1.7 & 0.1 & 20.3 & 8.1 & 0.2 & 8.04 & 0.24 \\
\hline $\mathrm{R} 2$ & 50 & 616 & 27,622 & 1,781 & 0.0 & 0.0 & 12.9 & 2.4 & 0.2 & 7.37 & 0.20 \\
\hline $\mathrm{RC} 1$ & 61 & 793 & 17,813 & 3,633 & 1.0 & 0.1 & 45.6 & 3.7 & 1.1 & 7.25 & 0.30 \\
\hline $\mathrm{RC} 2$ & 61 & 745 & 13,556 & 3,653 & 0.0 & 0.1 & 31.0 & 1.3 & 2.1 & 5.75 & 0.36 \\
\hline Tot./Avg. & 336 & 678 & 15,888 & 3,151 & 0.7 & 0.1 & 32.8 & 3.3 & 1.0 & 6.75 & 0.30 \\
\hline
\end{tabular}


Table EC.17 Impact of inconvenience constraints on algorithm performance for objective II, grouped by Solomon class

\begin{tabular}{|c|c|c|c|c|c|c|c|c|c|c|c|}
\hline \multirow[b]{2}{*}{$\begin{array}{r}\text { Policy / } \\
\text { Solomon } \\
\text { class }\end{array}$} & \multirow[b]{2}{*}{$\begin{array}{l}\text { Num. } \\
\text { opt. } \\
\text { sols. }\end{array}$} & \multicolumn{10}{|c|}{ Average of } \\
\hline & & $\begin{array}{l}\text { Run } \\
\text { time }\end{array}$ & $\begin{array}{c}\text { B\&B } \\
\text { nodes }\end{array}$ & $\begin{array}{l}\text { Cap. } \\
\text { cuts }\end{array}$ & $\begin{array}{c}\text { 2-path } \\
\text { cuts }\end{array}$ & $\begin{array}{c}\text { Conn. } \\
\text { cuts }\end{array}$ & $\begin{array}{l}\text { Inf.- } \\
\text { path. } \\
\text { cuts }\end{array}$ & $\begin{array}{l}\text { Path- } \\
\text { match. } \\
\text { cuts }\end{array}$ & $\begin{array}{c}\text { Feas. } \\
\text { cuts }\end{array}$ & $\begin{array}{l}\text { Relative } \\
\text { separation } \\
\text { strength. } \\
\text { cuts }\end{array}$ & $\begin{array}{l}\text { time } \\
\text { feas. } \\
\text { cuts }\end{array}$ \\
\hline \multicolumn{12}{|l|}{ SDVRPTW } \\
\hline $\mathrm{C} 1$ & 41 & 288 & 3,114 & 2,692 & 3.3 & 0.0 & 10.4 & 0.4 & 0.0 & 5.18 & 0.41 \\
\hline $\mathrm{C} 2$ & 38 & 169 & 2,656 & 1,819 & 0.0 & 0.0 & 13.5 & 0.7 & 0.0 & 4.80 & 1.05 \\
\hline R1 & 58 & 264 & 12,074 & 1,661 & 2.4 & 0.1 & 22.0 & 11.1 & 42.4 & 7.77 & 0.50 \\
\hline $\mathrm{R} 2$ & 46 & 438 & 16,467 & 1,901 & 0.0 & 0.3 & 20.9 & 7.7 & 212.2 & 6.43 & 0.47 \\
\hline $\mathrm{RC} 1$ & 64 & 278 & 4,545 & 2,162 & 4.7 & 0.8 & 30.2 & 6.6 & 13.5 & 8.64 & 1.23 \\
\hline $\mathrm{RC} 2$ & 40 & 126 & 3,551 & 1,462 & 0.0 & 1.1 & 26.4 & 11.5 & 70.0 & 6.99 & 1.48 \\
\hline Tot./Avg. & 287 & 266 & 7,384 & 1,951 & 2.0 & 0.4 & 21.5 & 6.7 & 55.3 & 6.88 & 0.85 \\
\hline \multicolumn{12}{|l|}{ NV2 } \\
\hline $\mathrm{C} 2$ & 38 & 152 & 2,727 & 1,837 & 0.1 & 0.0 & 13.1 & 0.3 & 0.0 & 4.46 & 2.27 \\
\hline R1 & 56 & 401 & 15,100 & 1,868 & 2.8 & 0.1 & 21.2 & 9.5 & 67.5 & 7.31 & 0.52 \\
\hline $\mathrm{R} 2$ & 43 & 711 & 22,016 & 2,230 & 0.0 & 0.2 & 19.3 & 6.4 & 233.2 & 5.93 & 0.52 \\
\hline $\mathrm{RC} 1$ & 64 & 260 & 4,308 & 2,202 & 4.2 & 0.6 & 21.6 & 4.4 & 10.8 & 8.62 & 0.96 \\
\hline $\mathrm{RC} 2$ & 40 & 173 & 4,245 & 1,549 & 0.0 & 1.1 & 24.8 & 8.0 & 72.3 & 7.64 & 0.92 \\
\hline Tot./Avg. & 281 & 339 & 8,932 & 2,067 & 2.0 & 0.3 & 18.8 & 5.2 & 63.5 & 6.69 & 0.89 \\
\hline \multicolumn{12}{|l|}{ So } \\
\hline $\mathrm{C} 1$ & 40 & 402 & 3,341 & 2,821 & 4.4 & 0.0 & 12.6 & 0.2 & 0.1 & 4.24 & 0.36 \\
\hline $\mathrm{C} 2$ & 37 & 252 & 2,397 & 1,961 & 0.2 & 0.0 & 14.1 & 0.5 & 0.0 & 3.68 & 1.08 \\
\hline R1 & 50 & 855 & 22,405 & 2,237 & 3.4 & 0.1 & 34.8 & 22.4 & 324.9 & 6.40 & 0.77 \\
\hline $\mathrm{R} 2$ & 24 & 1,954 & 37,389 & 2,747 & 0.0 & 0.6 & 40.0 & 29.8 & $1,327.9$ & 4.08 & 1.76 \\
\hline $\mathrm{RC} 1$ & 52 & 883 & 11,575 & 2,443 & 5.3 & 0.6 & 45.7 & 9.1 & 550.4 & 7.25 & 1.35 \\
\hline $\mathrm{RC} 2$ & 28 & 1,169 & 18,017 & 1,940 & 0.0 & 2.7 & 78.0 & 60.3 & $1,108.2$ & 4.78 & 2.03 \\
\hline Tot./Avg. & 231 & 937 & 16,408 & 2,370 & 2.5 & 0.6 & 38.2 & 19.8 & 555.7 & 5.32 & 1.22 \\
\hline \multicolumn{12}{|l|}{ TNV25 } \\
\hline $\mathrm{C} 1$ & 41 & 136 & 1,633 & 2,276 & 4.2 & 0.0 & 4.0 & 0.0 & 0.0 & 5.25 & 0.65 \\
\hline $\mathrm{R} 2$ & 34 & 1,239 & 16,925 & 6,160 & 0.0 & 0.0 & 16.9 & 1.0 & 0.1 & 4.02 & 0.07 \\
\hline $\mathrm{RC} 1$ & 48 & 1,185 & 7,844 & 5,987 & 6.2 & 0.0 & 37.1 & 0.5 & 0.0 & 6.25 & 0.10 \\
\hline $\mathrm{RC} 2$ & 28 & 1,186 & 10,076 & 5,825 & 0.0 & 0.3 & 48.6 & 5.3 & 55.0 & 5.03 & 0.23 \\
\hline Tot./Avg. & 235 & 878 & 9,108 & 4,829 & 2.7 & 0.0 & 23.5 & 1.5 & 8.2 & 5.07 & 0.24 \\
\hline \multicolumn{12}{|l|}{ TNV50 } \\
\hline $\mathrm{C} 1$ & 41 & 134 & 1,654 & 2,381 & 4.2 & 0.0 & 4.5 & 0.0 & 0.0 & 5.03 & 0.47 \\
\hline $\mathrm{C} 2$ & 38 & 87 & 1,764 & 1,698 & 0.1 & 0.0 & 5.0 & 0.0 & 0.0 & 4.49 & 1.03 \\
\hline $\mathrm{R} 1$ & 49 & 954 & 19,249 & 4,361 & 3.2 & 0.0 & 32.0 & 5.5 & 3.0 & 5.89 & 0.23 \\
\hline $\mathrm{R} 2$ & 36 & 1,168 & 24,140 & 4,242 & 0.0 & 0.0 & 23.5 & 3.8 & 29.9 & 4.72 & 0.46 \\
\hline $\mathrm{RC} 1$ & 49 & 1,049 & 7,665 & 4,955 & 6.0 & 0.1 & 39.8 & 1.2 & 0.0 & 6.98 & 0.18 \\
\hline $\mathrm{RC} 2$ & 29 & 1,053 & 10,718 & 4,521 & 0.0 & 0.5 & 54.7 & 7.2 & 56.2 & 5.52 & 0.27 \\
\hline Tot./Avg. & 242 & 791 & 11,432 & 3,861 & 2.6 & 0.1 & 28.1 & 3.0 & 13.2 & 5.59 & 0.40 \\
\hline \multicolumn{12}{|l|}{ TNV75 } \\
\hline $\mathrm{C} 1$ & 41 & 139 & 1,654 & 2,381 & 4.2 & 0.0 & 4.5 & 0.0 & 0.0 & 4.97 & 0.47 \\
\hline $\mathrm{C} 2$ & 38 & 96 & 1,955 & 1,735 & 0.1 & 0.0 & 7.0 & 0.0 & 0.0 & 4.66 & 0.98 \\
\hline $\mathrm{R} 1$ & 50 & 893 & 28,171 & 2,859 & 2.5 & 0.0 & 31.3 & 8.3 & 7.8 & 6.34 & 0.35 \\
\hline $\mathrm{R} 2$ & 40 & 912 & 34,046 & 2,618 & 0.1 & 0.1 & 23.1 & 4.8 & 30.8 & 5.43 & 0.48 \\
\hline $\mathrm{RC} 1$ & 54 & 898 & 11,625 & 3,753 & 5.6 & 0.1 & 41.1 & 2.1 & 0.2 & 7.35 & 0.19 \\
\hline $\mathrm{RC} 2$ & 33 & 815 & 11,520 & 3,202 & 0.0 & 0.5 & 41.5 & 8.6 & 15.0 & 5.83 & 0.32 \\
\hline Tot./Avg. & 256 & 673 & 15,843 & 2,850 & 2.4 & 0.1 & 26.6 & 4.1 & 8.7 & 5.93 & 0.43 \\
\hline
\end{tabular}


Table EC.18 Impact of inconvenience constraints on algorithm performance for objective III, grouped by Solomon class

\begin{tabular}{|c|c|c|c|c|c|c|c|c|c|c|c|}
\hline \multirow[b]{2}{*}{$\begin{array}{r}\text { Policy / } \\
\text { Solomon } \\
\text { class }\end{array}$} & \multirow[b]{2}{*}{$\begin{array}{l}\text { Num. } \\
\text { opt. } \\
\text { sols. }\end{array}$} & \multicolumn{10}{|c|}{ Average of } \\
\hline & & $\begin{array}{l}\text { Run } \\
\text { time }\end{array}$ & $\begin{array}{c}\text { B\&B } \\
\text { nodes }\end{array}$ & $\begin{array}{l}\text { Cap. } \\
\text { cuts }\end{array}$ & $\begin{array}{c}\text { 2-path } \\
\text { cuts }\end{array}$ & $\begin{array}{c}\text { Conn. } \\
\text { cuts }\end{array}$ & $\begin{array}{l}\text { Inf.- } \\
\text { path. } \\
\text { cuts }\end{array}$ & $\begin{array}{l}\text { Path- } \\
\text { match. } \\
\text { cuts }\end{array}$ & $\begin{array}{c}\text { Feas. } \\
\text { cuts }\end{array}$ & $\begin{array}{r}\text { Re } \\
\text { separa } \\
\text { streng } \\
\text { cuts }\end{array}$ & $\begin{array}{l}\text { ive } \\
\text { n time } \\
\text { feas. } \\
\text { cuts }\end{array}$ \\
\hline \multicolumn{12}{|l|}{ SDVRPTW } \\
\hline $\mathrm{C} 1$ & 31 & 343 & 6,082 & 2,758 & 5.7 & 0.0 & 25.4 & 3.1 & 3.2 & 5.37 & 0.74 \\
\hline $\mathrm{C} 2$ & 29 & 205 & 4,704 & 1,872 & 0.6 & 0.1 & 32.6 & 5.3 & 8.1 & 5.42 & 1.76 \\
\hline R1 & 56 & 318 & 12,788 & 2,077 & 3.1 & 0.1 & 30.0 & 28.5 & 47.6 & 7.58 & 2.86 \\
\hline $\mathrm{R} 2$ & 44 & 530 & 18,830 & 2,235 & 0.1 & 0.1 & 28.8 & 16.6 & 293.0 & 5.73 & 3.86 \\
\hline $\mathrm{RC} 1$ & 59 & 224 & 5,760 & 2,105 & 3.0 & 0.5 & 32.9 & 7.0 & 14.6 & 8.87 & 3.26 \\
\hline $\mathrm{RC} 2$ & 35 & 520 & 7,179 & 1,567 & 0.0 & 1.4 & 42.9 & 20.1 & 176.8 & 6.45 & 14.41 \\
\hline Tot./Avg. & 254 & 351 & 9,688 & 2,100 & 2.2 & 0.4 & 32.0 & 14.5 & 90.3 & 6.89 & 4.33 \\
\hline \multicolumn{12}{|l|}{ NV2 } \\
\hline $\mathrm{C} 2$ & 29 & 334 & 5,066 & 2,044 & 0.7 & 0.1 & 26.4 & 1.9 & 2.6 & 5.77 & 1.28 \\
\hline R1 & 54 & 467 & 14,244 & 2,511 & 3.3 & 0.1 & 29.1 & 19.2 & 26.5 & 6.98 & 0.77 \\
\hline $\mathrm{R} 2$ & 38 & 1,011 & 26,581 & 3,109 & 0.1 & 0.2 & 29.8 & 13.8 & 342.4 & 5.03 & 3.57 \\
\hline $\mathrm{RC} 1$ & 59 & 215 & 5,794 & 2,094 & 3.1 & 0.4 & 28.6 & 6.5 & 13.5 & 9.28 & 1.02 \\
\hline $\mathrm{RC} 2$ & 34 & 289 & 5,780 & 1,644 & 0.0 & 1.3 & 32.2 & 13.3 & 160.1 & 6.48 & 11.74 \\
\hline Tot./Avg. & 245 & 442 & 11,067 & 2,355 & 2.1 & 0.3 & 27.5 & 10.3 & 90.8 & 6.81 & 2.83 \\
\hline \multicolumn{12}{|l|}{ So } \\
\hline $\mathrm{C} 1$ & 30 & 348 & 4,977 & 2,687 & 5.6 & 0.1 & 24.5 & 2.8 & 4.5 & 4.56 & 0.39 \\
\hline $\mathrm{C} 2$ & 27 & 493 & 9,264 & 1,784 & 0.5 & 1.1 & 63.8 & 32.8 & 336.1 & 4.68 & 2.56 \\
\hline R1 & 45 & 1,120 & 26,166 & 3,073 & 5.4 & 0.2 & 66.1 & 63.1 & 323.3 & 5.64 & 1.67 \\
\hline $\mathrm{R} 2$ & 19 & 2,331 & 35,776 & 3,248 & 0.2 & 0.6 & 69.0 & 55.7 & $1,179.3$ & 2.94 & 17.06 \\
\hline $\mathrm{RC} 1$ & 45 & 1,098 & 14,876 & 2,537 & 3.5 & 0.6 & 68.1 & 19.9 & 503.7 & 7.21 & 3.85 \\
\hline $\mathrm{RC} 2$ & 21 & 1,687 & 26,094 & 2,201 & 0.0 & 5.2 & 153.5 & 102.1 & $1,257.9$ & 3.88 & 12.25 \\
\hline Tot./Avg. & 187 & 1,237 & 20,683 & 2,664 & 2.8 & 1.1 & 73.8 & 46.3 & 604.8 & 5.06 & 6.25 \\
\hline \multicolumn{12}{|l|}{ TNV25 } \\
\hline $\mathrm{C} 1$ & 30 & 292 & 2,780 & 3,502 & 5.8 & 0.0 & 7.2 & 0.0 & 0.0 & 4.41 & 0.16 \\
\hline $\mathrm{R} 2$ & 15 & 2,632 & 15,984 & 15,434 & 0.0 & 0.0 & 28.6 & 1.7 & 0.3 & 2.38 & 0.01 \\
\hline $\mathrm{RC} 1$ & 31 & 1,915 & 10,808 & 11,334 & 7.4 & 0.0 & 49.5 & 2.5 & 0.0 & 4.56 & 0.15 \\
\hline $\mathrm{RC} 2$ & 19 & 1,679 & 14,622 & 8,021 & 0.0 & 0.3 & 52.8 & 6.9 & 3.1 & 4.16 & 0.08 \\
\hline Tot./Avg. & 145 & 1,722 & 10,486 & 10,666 & 3.8 & 0.0 & 34.8 & 3.3 & 2.1 & 3.90 & 0.14 \\
\hline \multicolumn{12}{|l|}{ TNV50 } \\
\hline $\mathrm{C} 1$ & 30 & 282 & 3,076 & 3,561 & 5.6 & 0.0 & 9.4 & 0.1 & 0.3 & 4.69 & 0.13 \\
\hline $\mathrm{C} 2$ & 25 & 755 & 5,890 & 5,380 & 0.9 & 0.0 & 23.8 & 0.7 & 8.4 & 4.59 & 0.33 \\
\hline $\mathrm{R} 1$ & 26 & 2,123 & 14,915 & 14,397 & 6.1 & 0.0 & 64.5 & 18.1 & 3.5 & 4.31 & 0.08 \\
\hline $\mathrm{R} 2$ & 20 & 2,296 & 17,501 & 14,843 & 0.1 & 0.0 & 44.8 & 5.4 & 46.1 & 2.92 & 0.09 \\
\hline $\mathrm{RC} 1$ & 33 & 1,724 & 8,951 & 11,195 & 7.5 & 0.1 & 63.3 & 3.6 & 0.1 & 4.79 & 0.25 \\
\hline $\mathrm{RC} 2$ & 22 & 1,352 & 11,113 & 7,528 & 0.1 & 0.5 & 65.5 & 11.4 & 104.0 & 4.98 & 0.11 \\
\hline Tot./Avg. & 156 & 1,573 & 10,978 & 10,432 & 3.9 & 0.1 & 49.6 & 7.4 & 24.1 & 4.35 & 0.16 \\
\hline \multicolumn{12}{|l|}{ TNV75 } \\
\hline $\mathrm{C} 1$ & 29 & 328 & 3,087 & 3,368 & 5.6 & 0.0 & 9.5 & 0.1 & 0.3 & 4.60 & 0.17 \\
\hline $\mathrm{C} 2$ & 25 & 760 & 6,317 & 4,741 & 0.9 & 0.0 & 24.1 & 0.9 & 2.3 & 4.65 & 0.54 \\
\hline $\mathrm{R} 1$ & 32 & 1,733 & 21,690 & 8,887 & 5.0 & 0.0 & 65.8 & 24.5 & 12.2 & 4.99 & 0.27 \\
\hline $\mathrm{R} 2$ & 24 & 1,999 & 21,254 & 10,400 & 0.0 & 0.0 & 46.2 & 9.3 & 48.9 & 3.41 & 0.14 \\
\hline $\mathrm{RC} 1$ & 44 & 1,208 & 14,770 & 6,377 & 5.9 & 0.1 & 48.2 & 3.6 & 1.0 & 6.06 & 0.23 \\
\hline $\mathrm{RC} 2$ & 29 & 675 & 8,133 & 4,331 & 0.1 & 0.9 & 53.5 & 10.8 & 49.7 & 5.51 & 0.33 \\
\hline Tot./Avg. & 183 & 1,229 & 14,114 & 6,791 & 3.3 & 0.2 & 45.0 & 9.4 & 18.5 & 4.95 & 0.27 \\
\hline
\end{tabular}

